\title{
HARMONIC AND QUASI-HARMONIC SPHERES, PART III. RECTIFIABLITY OF THE PARABOLIC DEFECT MEASURE AND GENERALIZED VARIFOLD FLOWS
}

\author{
FangHua LIN ${ }^{\text {a, } 1}$, ChangYou WANG ${ }^{\text {b,2 }}$ \\ ${ }^{a}$ Courant Institute of Mathematical Sciences, New York University, New York, NY 10012, USA \\ ${ }^{\mathrm{b}}$ Department of Mathematics, University of Kentucky, Lexington, KY 40506, USA
}

Received 2 May 2000, revised 23 July 2001

\begin{abstract}
We study weakly convergent sequences of suitable weak solutions of heat flows of harmonic maps or approximated harmonic maps. We prove a dimensional stratification for the space-time concentration measure and verify that the concentration measure, viewed as a generalized varifold, moves according to the generalized varifold flow rule which reduces to the Brakke's flow of varifold provided that the limiting harmonic map flow is suitable. We also establish an energy quantization for the density of the limiting varifold.

(C) 2002 L'Association Publications de l'Institut Henri Poincaré. Published by Elsevier B.V. All rights reserved

AMS classification: $35 \mathrm{~K} 55 ; 58 \mathrm{~J} 35$

Keywords: Harmonic or approximated harmonic map flows; Concentration measures; Stratification; Rectifiablity; Generalized varifold flows; Brakke's flow; Energy quantization

RÉSUMÉ. - Nous étudions des séquences faiblement convergentes de solutions faibles du flot de chaleur d'applications harmoniques (éventuellement approximées). Nous prouvons une stratification dimensionnelle pour la mesure de concentration de l'espace-temps et vérifions que la mesure de concentration, vue comme une varifold generalisée, est sujette à la règle du flot généralisé des varifolds qui se réduit à la règle du flot de Brakke pour autant que l'application harmonique soit adéquate. Nous établissons aussi une quantification de l'énergie pour la densité de de la varifold limite de la séquence.
\end{abstract}

(C) 2002 L'Association Publications de l'Institut Henri Poincaré. Published by Elsevier B.V. All rights reserved

\section{Introduction}

This is the third part of our project initiated in Lin and Wang [31] on the study of the weakly convergent sequence of smooth (or certain classes of weak) solutions to the heat equation of harmonic maps or approximated harmonic maps (i.e. the negative gradient flow of the generalized Ginzburg-Landau functionals). The general situation for the

\footnotetext{
E-mail addresses: linf@cims.nyu.edu (F. Lin), cywang@ms.uky.edu (C. Wang).

${ }^{1}$ Supported in part by NSF DMS 9896391.

${ }^{2}$ Supported in part by NSF DMS 9970549 and an AMS Centennial Fellowship.
} 
heat flow of harmonic maps is as follows. Let $u_{n}(x, t): M \times R_{+} \rightarrow N$ be a sequence of smooth solutions to the heat flow of harmonic maps from a $m$-dimensional compact smooth Riemannian manifold $M$ (with possibly nonempty smooth boundary $\partial M$ ) into a compact smooth Riemannian manifold $N \subset R^{k}$ without boundary, namely,

$$
\partial_{t} u_{n}-\Delta u_{n}=A\left(u_{n}\right)\left(D u_{n}, D u_{n}\right), \quad \text { in } M \times R_{+}
$$

where $A(\cdot)(\cdot, \cdot)$ denotes the second fundamental form of $N$ in $R^{k}$, such that $u_{n}(x, t)$ weakly converges to $u(x, t)$ in $H_{\mathrm{loc}}^{1}\left(M \times R_{+}, R^{k}\right)$. By the Fatou's lemma, we may assume that

$$
\frac{1}{2}\left|D u_{n}\right|^{2}(x, t) \mathrm{d} x \mathrm{~d} t \rightarrow \frac{1}{2}|D u|^{2}(x, t) \mathrm{d} x \mathrm{~d} t+v
$$

and

$$
\left|\partial_{t} u_{n}\right|^{2}(x, t) \mathrm{d} x \mathrm{~d} t \rightarrow\left|\partial_{t} u\right|^{2}(x, t) \mathrm{d} x \mathrm{~d} t+\eta
$$

as convergence of Radon measures on $M \times R_{+}$for some nonnegative Randon measures $v, \eta$ supported on the so-called energy concentration set $\Sigma \subset M \times R_{+}$(see [31]). It is easy to check that $v=v_{t} \mathrm{~d} t$ for some nonnegative Radon measures $v_{t}, t \in R_{+}$(see, Lemma 2.5 below). The main result of [31] is (see Lin [23] for the static cass) to characterize the necessary and sufficient conditions, under which both $v$ and $\eta$ vanish, in terms of the existence (or non-existence) of harmonic and quasi-harmonic spheres into $N$. In other words, the necessary and sufficient conditions for such weakly convergent sequences to be strongly convergent. As a consequence of such a characterization is a new proof of the classical theorem by Eells and Sampson [15] (without the nonpositive curvature condition on $N$ ) under a new set of necessary and sufficient conditions (cf. [31]). In general, we showed in [31] that, without any extra assumption on $N$, both $v$ and $\eta$ are supported on the energy concentration set $\Sigma$, which is closed and has locally finite $m$-dimensional Hausdorff measure, $\mathcal{P}^{m}$, with respect to the parabolic metric on $M \times R_{+}$, and for $\mathcal{P}^{m}$ almost all such points in the energy concentration set $\Sigma$, one has the $m$-dimensional density of $v$ (with respect to the parabolic metric) strictly positive and finite. Moreover, for $L^{1}$ a.e. $t \in R_{+}, v_{t}$ has the $(m-2)$-dimensional density (with respect to the Euclidean metric on $M$ ) positive and finite for $H^{m-2}$ a.e. $x \in M$. In fact, it was shown by Cheng [9] that for all $t \in R_{+}$, the support of $v_{t}$ has locally finite $(m-2)$-dimensional Hausdorff measure. It is not very hard to generalize the argument of Lin [23] to show that for $L^{1}$ a.e. $t \in R_{+}, \Sigma_{t}=\Sigma \cap\{t\}$ and the support of $v_{t}$ are $(m-2)$-rectifiable. Here we shall adopt a different and conceptually much easier approach in Section 4, namely the generalized varifold approach which is a natural extension of the classical varifold concept of Almgren [3,4] and Allard [6]. Roughly, we associate each $u_{n}$ with a $(m-2)$-generalized varifold $V_{u_{n}}$ on $M \times R_{+}$and show that $V_{u_{n}}$ converges to a $(m-2)$-generalized varifold $V=V_{t} \mathrm{~d} t, V_{t}$ has its generalized mean curvature $H_{t} \in L_{\left\|V_{t}\right\|}^{2}\left(M, R^{m}\right)$ for $L^{1}$ a.e. $t \in R_{+}$, and then the extension of Allard's rectifiablity result from classical varifolds to generalized varifolds yields that $V_{t} \mathrm{~L}(\{x \in$ $\left.\left.M: \Theta^{m-2}\left(\left\|V_{t}\right\|, x\right)>0\right\}\right)$ is $(m-2)$-rectifiable. This rectifiablity result of $\Sigma_{t}$ was also proved in a recent paper of Li and Tian [29] for so-called strongly stationary weakly heat flow of harmonic maps which are weak solutions of the heat flow (1.0) with energy 
monotonicity, energy inequality, and the small energy regularity properties, where they verified the condition of Preiss's rectifiablity theorem. As pointed out in [31], although all the analysis in the present article and in [31,32] are for smooth solutions of the heat equation of harmonic maps (or solutions to the gradient flow of the Ginzburg-Landau functionals), the facts that we need are exactly these three properties stated above (see also Section 7 below), therefore all the results of the present article and [31,32] remain to be true for the class of weak solutions to the heat flow of harmonic maps satisfying these three properties. For simplicity of descriptions, we will work for solutions to heat flows of the Ginzburg-Landau functionals only and state some analogous conclusions in Section 7 for this class of weak solutions of heat flows for harmonic maps.

One of the main results of the present paper is to show the pair $\left(u, v_{t} \mathrm{~d} t\right)$ satisfies the so-called generalized varifold flow (see Definition 5.5 of Section 5 below for the definition), as in the recent very interesting work by Ambrosio and Soner [1]. Moreover, in the case that $u$ is a suitable weak solution (e.g. $u$ is smooth), i.e. satisfying the standard energy equality in both local and global forms, then $\left\{v_{t}\right\}_{t \geqslant 0}$ is a Brakke flow of (rectifiable) varifolds, i.e. flow by the mean curvature in the varifold sense defined by Brakke [8]. We point out that a weaker version of this fact was also shown by [29] where a factor $\frac{1}{2}$ is putted in front of the mean curvature square term of the energy inequality (5.7). To improve the factor $\frac{1}{2}$ to 1 and to establish that the flow is actually the Brakke flow is one of the most difficult analytical points in all such related analysis (see also discussions in [1], in particular $\$ 6$ of [1]). To achieve such a goal, one method is to establish the local almost conformal property of the solution map restricted to the 2-dimensional plane orthogonal to the tangent plane of the energy concentration set $\Sigma$ (see Section 5 below). We also establish in Theorem 6.7 the energy quantization result in dimension large than two, which extends the main result of our part II [32]. Note that, in the case $N=S^{k-1}$, Theorem 6.7 can be used to give an alternative proof of the improvement of $\frac{1}{2}$ to 1 . However, the argument of Section 5 is independent of $N$. The energy quantization in dimension at least 3 was first established by Lin and Rivieré [27] for stationary harmonic maps into spheres, by Lin and Wang [28] for approximated harmonic maps, and by Lin and Rivieré [28] for Ginzburg-Landau vortices in $R^{3}$. Our result here can be viewed as parabolic version of $[27,32]$.

Since we can treat smooth solutions to the heat flow of harmonic maps in almost the same way as that of the heat equation of the generalized Ginzburg-Landau functional. For simplicity, we will present our result in the context of solutions to the heat flow of generalized Ginzburg-Landau functional and make remarks concerning the heat flow of harmonic maps in Section 7. Now let us describe precisely the results and the structure of the present article.

For $\varepsilon>0$, we consider the (generalized) Ginzburg-Landau functional

$$
I_{\varepsilon}(u)=\int_{M}\left(\frac{1}{2}|D u|^{2}+\frac{1}{\varepsilon^{2}} F(u)\right) \mathrm{d} x
$$


where $F \in C^{\infty}\left(R^{k}, R\right)$ satisfies:

$$
F(p)= \begin{cases}d^{2}(p, N), & \text { if } d(p, N) \leqslant \delta \\ 4 \delta^{2}, & \text { if } d(p, N) \geqslant 2 \delta\end{cases}
$$

Here $d$ denotes the Euclidean distance in $R^{k}$ and $d(\cdot, N)=\inf \{d(\cdot, p): p \in N\}$. Note that $\delta>0$ is chosen to be so small that $d^{2}(p, N)$ is smooth for $p \in N_{2 \delta} \equiv\{p: d(p, N) \leqslant$ $2 \delta\}$. Let $u_{\varepsilon} \in C^{\infty}\left(M \times R_{+}, R^{k}\right)$ be solutions to the heat equation

$$
\begin{aligned}
\partial_{t} u_{\varepsilon}-\Delta u_{\varepsilon} & =\frac{1}{\varepsilon^{2}} f\left(u_{\varepsilon}\right) \quad(x, t) \in M \times R_{+} \\
u_{\varepsilon}(x, 0) & =u_{0}(x), \quad x \in M,
\end{aligned}
$$

where $f\left(u_{\varepsilon}\right)=-(D F)\left(u_{\varepsilon}\right)$ and $u_{0} \in C^{2}(M, N)$ is a given map. We assume throughout this paper that if $N=S^{k-1}$ then $F(p)=\frac{1}{4}\left(1-|p|^{2}\right)^{2}$ so that $f(p)=p\left(1-|p|^{2}\right)$ and (1.4) becomes

$$
\partial_{t} u_{\varepsilon}-\Delta u_{\varepsilon}=\frac{1}{\varepsilon^{2}} u_{\varepsilon}\left(1-\left|u_{\varepsilon}\right|^{2}\right) .
$$

It is easy to see that $u_{\varepsilon}$ satisfies the following energy equality (see also Lemma 2.1 below):

$$
\sup _{0<t<\infty}\left(\int_{0}^{t} \int_{M}\left|\partial_{t} u_{\varepsilon}\right|^{2} \mathrm{~d} x \mathrm{~d} t+I_{\varepsilon}\left(u_{\varepsilon}(\cdot, t)\right)\right)=\frac{1}{2} \int_{M}\left|D u_{0}\right|^{2} .
$$

It follows from (1.6) that for any $\varepsilon \downarrow 0$ there exists a subsequence $\varepsilon_{n} \rightarrow 0$ such that $u_{n} \equiv u_{\varepsilon_{n}} \rightarrow u$ weakly in $H_{\text {loc }}^{1}\left(M \times R_{+}, R^{k}\right)$. Moreover, it was shown by Chen and Struwe [13] (for $\partial M=\emptyset$ ) and Chen and Lin [11] (for $\partial M \neq \emptyset$ ) that there exists a closed subset $\Sigma=\left\{\left(\Sigma_{t}, t\right): t>0\right\} \subset M \times R_{+}$(see the definition of $\Sigma$ in Section 2 below) such that $u_{\varepsilon_{n}} \rightarrow u$ in $H_{\mathrm{loc}}^{1} \cap C_{\mathrm{loc}}^{1}\left(M \times R_{+} \backslash \Sigma, R^{k}\right)$. In particular, $u \in C^{\infty}\left(M \times R_{+} \backslash \Sigma, N\right)$ is a smooth solution to the harmonic map flow equation (1.0). Let

$$
e_{\varepsilon_{n}}\left(u_{n}\right)(x, t)=\frac{1}{2}\left|D u_{n}\right|^{2}(x, t)+\frac{1}{\varepsilon_{n}^{2}} F\left(u_{n}\right)(x, t) .
$$

We will simply write $e\left(u_{n}\right)$ for $e_{\varepsilon_{n}}\left(u_{n}\right)$ through this paper as long as there is no ambiguity. We can assume that

$$
e\left(u_{n}\right)(x, t) \mathrm{d} x \mathrm{~d} t \rightarrow \frac{1}{2}|D u|^{2}(x, t) \mathrm{d} x \mathrm{~d} t+v
$$

and

$$
\left|\partial_{t} u_{n}\right|^{2}(x, t) \mathrm{d} x \mathrm{~d} t \rightarrow\left|\partial_{t} u\right|^{2}(x, t) \mathrm{d} x \mathrm{~d} t+\eta
$$

as convergence of Radon measures for two nonnegative Radon measures $v$ and $\eta$ on $M \times R_{+}$.

The stratification for the singular set of area minimizing currents was studied by Federer [16], who introduced a powerful scheme called the Federer's dimension 
reduction argument (see also Almgren [3] and the appendix of [33]). White [37] provided an abstract approach to obtain the stratification for a large class of variational problems varying from minimizing currents, energy minimizing harmonic maps, and mean curvature flows (see also [34] for the stratification for minimizing harmonic maps). Motivated by these stratification results, we carry out the stratification for the parabolic concentration set $\Sigma$ in Section 3. The stratification Theorem 3.6 roughly says that the subset $\Sigma_{k}$, consisting of $z_{0} \in \Sigma$ such that the $\mathcal{P}_{\lambda}$-invariant subspace of any tangent measure $\mu^{0}$ of $\mu$ at $z_{0}$ has its dimension of vector space at most $k$, has Hausdorff dimension measured in the parabolic metric at most $k$, for $0 \leqslant k \leqslant m$.

In Section 4, we adopt the generalized $(m-2)$-varifold concept, which is a natural and very useful extension of the classical varifold concept studied by Almgren $[3,4]$ and Allard [6], to study the convergence problem in our case through a varifold approach. Once we associate a sequence of generalized $(m-2)$-varifolds, $V_{u_{n}}$, for $u_{n}$ 's, we can consider the limiting generalized $(m-2)$-varifold $V=V_{t} \mathrm{~d} t$ of $V_{u_{n}}$. In Section 4, we show that for $L^{1}$ a.e. $t>0$, the first variation of $V_{t}, \delta V_{t}$, is a Radon measure which is absolutely continuous with respect to $\left\|V_{t}\right\|$ and its generalized measure curvature

$$
H_{t}=\frac{\delta V_{t}}{\left\|V_{t}\right\|} \in L_{\left\|V_{t}\right\|}^{2}\left(M, R^{m}\right) \text {. }
$$

Therefore, $\Theta^{m-2}\left(\left\|V_{t}\right\|, \cdot\right)$ exists for $H^{m-2}$ a.e. in $M$. We then show in Theorem 4.9 that $V_{t} \mathrm{~L}\left\{x \in M: 0<\Theta^{m-2}\left(\left\|V_{t}\right\|, x\right)<\infty\right\}$ is a $(m-2)$-rectifiable varifold. This, combined with the observation that for $H^{m-2}$ a.e. $x \in \Sigma_{t} \Theta^{m-2}\left(\left\|V_{t}\right\|, x\right)$ is positive and finite, shows that $\Sigma_{t}$ is $(m-2)$-rectifiable.

In Section 5, we continue our discussion from Section 4 and show, in Theorem 5.6, that the pair $\left(u, v_{t} \mathrm{~d} t\right)$ satisfies a generalized varifold flow defined as in Definition 5.5 of Section 5. As a consequence of this generalized varifold flow, we show that if the limiting map $u$ is a suitable weak solution defined by Definition 5.9, which requires that $u$ satisfies the energy equality (both locally and globally), then the defect measure $\left\{v_{t}\right\}_{t} \geqslant 0$ is in fact a Brakke flow, i.e.,

$$
\bar{D}_{t} v_{t}(\phi)=\limsup _{s \rightarrow t} \frac{v_{s}(\phi)-v_{t}(\phi)}{s-t} \leqslant-\int_{M}\left(\phi\left|H_{t}\right|^{2}-\left\langle\left(T_{x} \Sigma_{t}\right)^{\perp} D \phi, H_{t}\right\rangle\right) \mathrm{d} v_{t}
$$

for any $t \geqslant 0$ and $\phi \in C_{0}^{2}\left(M, R_{+}\right)$. One way to obtain the generalized varifold flow is to apply the energy quantization Theorem 6.1, which however is only proved for sphere targets at this stage. In Lemma 5.8 of Section 5 , we provide another approach to improve the so-called factor $\frac{1}{2}$ to 1 .

In Section 6, we consider the density function $\Theta^{m-2}\left(\left\|V_{t}\right\|, \cdot\right)$. Under the extra assumption that $N=S^{k-1} \subset R^{k}$, we show a quantization result for $\Theta^{m-2}\left(\left\|V_{t}\right\|, \cdot\right)$ in Theorem 6.1 and Theorem 6.7, which roughly says that for almost all $z_{0}=\left(x_{0}, t_{0}\right) \in \Sigma$, $\Theta^{m-2}\left(\left\|V_{t_{0}}\right\|, x_{0}\right)$ is the sum of energies of finitely many harmonic $S^{2}$ 's. This type of result is obtained by estimating the norm of $D u_{n}$ in the Lorentz spaces $L^{2,1}$ and $L^{2, \infty}$, which is a highly nontrivial observation in dimension large than two and largely owns its origin from Lin and Rivieré [27]. 
Section 2 of the paper is devoted to the collection of some necessary facts needed later, and in Section 7 we make a few remarks concerning either smooth solutions to the heat flow of harmonic maps or the weak solutions satisfying energy inequality, energy monotonicity inequality, and the $\varepsilon_{0}$-regularity.

\section{Basic estimates}

This section is devoted to establishing some necessary facts needed for later sections. First let us recall some useful notations from $[11,13,35]$. Let $\delta$ denote the parabolic metric on $M \times R_{+}$defined by

$$
\delta((x, t),(y, s))=\max \{|x-y|, \sqrt{|t-s|}\}, \quad \forall(x, t),(y, s) \in M \times R_{+} .
$$

For $0 \leqslant l \leqslant m$, let $\mathcal{P}^{l}$ denote the $l$-dimensional Hausdorff measure on $M \times R_{+}$(or $R^{m} \times$ $R_{+}$) with respect to the parabolic metric $\delta$, and $H^{l}$ denotes the $l$-dimensional Hausdorff measure on $M$ (or $R^{m}$ ) with the Euclidean metric. For $z_{0}=\left(x_{0}, t_{0}\right) \in R^{m} \times R_{+}$, let $G_{z_{0}}$ denote the backward heat kernel:

$$
G_{z_{0}}(x, t)=\left(4 \pi\left(t_{0}-t\right)\right)^{-\frac{m}{2}} \exp \left(-\frac{\left|x-x_{0}\right|^{2}}{4\left(t_{0}-t\right)}\right), \quad x \in R^{m}, 0<t<t_{0} .
$$

Let $i(M)>0$ denote the injectivity radius of $M$. For $0<R<\left\{\frac{\sqrt{t_{0}}}{2}, i(M)\right\}$, let

$$
\begin{gathered}
S_{R}\left(z_{0}\right)=M \times\left\{t=t_{0}-R^{2}\right\}, \\
T_{R}\left(z_{0}\right)=M \times\left\{t \in R_{+}: t_{0}-4 R^{2} \leqslant t \leqslant t_{0}-R^{2}\right\},
\end{gathered}
$$

and

$$
P_{R}\left(z_{0}\right)=B_{R}\left(x_{0}\right) \times\left[t_{0}-R^{2}, t_{0}+R^{2}\right],
$$

where $B_{R}\left(x_{0}\right) \subset M$ denotes the geodesic ball with center $x_{0}$ and radius $R$.

For solutions $u_{\varepsilon} \in C^{\infty}\left(M \times R_{+}, R^{k}\right)$ to (1.3)-(1.4). Define two normalized energy quantities as follows.

$$
\begin{gathered}
\Psi\left(u_{\varepsilon}, z_{0}, R\right)=\int_{T_{R}\left(z_{0}\right)} \eta^{2}(x) e\left(u_{\varepsilon}\right)(x, t) G_{z_{0}}(x, t) \mathrm{d} x \mathrm{~d} t, \\
\Phi\left(u_{\varepsilon}, z_{0}, R\right)=R^{2} \int_{S_{R}\left(z_{0}\right)} \eta^{2}(x) e\left(u_{\varepsilon}\right)(x, t) G_{z_{0}}(x, t) \mathrm{d} x,
\end{gathered}
$$

for $0<R<\min \left\{\frac{\sqrt{t_{0}}}{2}, i(M)\right\}$, here $\eta \in C_{0}^{1}(M)$ satisfies that $\eta=1$ on $B_{r_{0}}\left(x_{0}\right), \eta=0$ outside $B_{2 r_{0}}\left(x_{0}\right)$, and $|D \eta| \leqslant \frac{2}{r_{0}}$.

Now we recall the energy inequality, energy monotonicity inequality, and small energy regularity from [13]. 
LEMmA 2.1 (Energy equality). - Let $u_{\varepsilon} \in C^{\infty}\left(M \times R_{+}, R^{k}\right)$ solve (1.3)-(1.4). Then we have, for any $\phi \in C_{0}^{1}\left(M, R_{+}\right)$and $0 \leqslant t_{1} \leqslant t_{2} \leqslant \infty$,

$$
\begin{aligned}
\int_{M} e\left(u_{\varepsilon}\right)\left(x, t_{1}\right) \phi(x) \mathrm{d} x-\int_{M} e\left(u_{\varepsilon}\right)\left(x, t_{2}\right) \phi(x) \mathrm{d} x \\
=\int_{t_{1}} \int_{M} \phi(x)\left|\partial_{t} u_{\varepsilon}\right|^{2}(x, t) \mathrm{d} x \mathrm{~d} t+\int_{t_{1}}^{t_{2}} \int_{M}^{t_{2}} D \phi(x) D u_{\varepsilon} \cdot \partial_{t} u_{\varepsilon} \mathrm{d} x \mathrm{~d} t .
\end{aligned}
$$

In particular,

$$
\int_{M} e\left(u_{\varepsilon}\right)\left(x, t_{1}\right) \mathrm{d} x-\int_{M} e\left(u_{\varepsilon}\right)\left(x, t_{2}\right) \mathrm{d} x=\int_{t_{1}}^{t_{2}} \int_{M}\left|\partial_{t} u_{\varepsilon}\right|^{2}(x, t) \mathrm{d} x \mathrm{~d} t .
$$

LEMMA 2.2 (Energy monotonicity inequality). - Let $u_{\varepsilon} \in C^{\infty}\left(M \times R_{+}, R^{k}\right)$ solve (1.3)-(1.4). Then

$$
\begin{gathered}
\Psi\left(u_{\varepsilon}, z_{0}, R_{1}\right)+c \int_{R_{1}}^{R_{2}} r^{-1} \int_{T_{r}\left(z_{0}\right)}\left(\eta^{2} \frac{\left|\left(x-x_{0}\right) \cdot D u_{\varepsilon}+2\left(t-t_{0}\right) \partial_{t} u_{\varepsilon}\right|^{2}}{\left|t_{0}-t\right|}+\eta^{2} \frac{F\left(u_{\varepsilon}\right)}{\varepsilon^{2}}\right) G_{z_{0}} \\
\leqslant \mathrm{e}^{C\left(R_{2}-R_{1}\right)} \Psi\left(u_{\varepsilon}, z_{0}, R_{2}\right)+C E_{0}\left(R_{2}-R_{1}\right) \\
\Phi\left(u_{\varepsilon}, z_{0}, R_{1}\right) \leqslant \mathrm{e}^{C\left(R_{2}-R_{1}\right)} \Phi\left(u_{\varepsilon}, z_{0}, R_{2}\right)+C E_{0}\left(R_{2}-R_{1}\right)
\end{gathered}
$$

for $z_{0} \in M \times R_{+}, 0<R_{1} \leqslant R_{2}<\min \left\{\frac{\sqrt{t_{0}}}{2}, i(M)\right\}$. Here $c, C>0$ depend only on $M, m, N$, and $E_{0}=\frac{1}{2} \int_{M}\left|D u_{\varepsilon}\right|^{2}(x, 0)$.

LEMma 2.3 ( $\varepsilon_{0}$-regularity). - There exist $\varepsilon_{0}, \delta_{0}, C_{0}>0$ such that if, for some $0<$ $R \leqslant \min \left\{\frac{\sqrt{t_{0}}}{2}, i(M)\right\}, \Psi\left(u_{\varepsilon}, z_{0}, R\right) \leqslant \varepsilon_{0}^{2}$, then

$$
\sup _{z \in P_{\delta_{0} R}\left(z_{0}\right)} e\left(u_{\varepsilon}\right)(z) \leqslant C_{0}\left(\delta_{0} R\right)^{-2} .
$$

For $\varepsilon_{n} \downarrow 0$, we assume that $u_{n} \equiv u_{\varepsilon_{n}} \rightarrow u$ weakly in $H_{\text {loc }}^{1}\left(M \times R_{+}, R^{k}\right)$. Then there exist two nonnegative Radon measures $v, \eta$ on $M \times R_{+}$such that

$$
\begin{gathered}
e\left(u_{n}\right)(x, t) \mathrm{d} x \mathrm{~d} t \rightarrow \frac{1}{2}|D u|^{2}(x, t) \mathrm{d} x \mathrm{~d} t+v \equiv \mu, \\
\left|\partial_{t} u_{n}\right|^{2}(x, t) \mathrm{d} x \mathrm{~d} t \rightarrow\left|\partial_{t} u\right|^{2}(x, t) \mathrm{d} x \mathrm{~d} t+\eta,
\end{gathered}
$$

as convergence of Radon measures on $M \times R_{+}$. Moreover, if we define the concentration set

$$
\Sigma=\bigcup_{0<R<r_{0}}\left\{z \in M \times R_{+}: \lim _{n \rightarrow \infty} \int_{T_{R}(z)} \eta^{2}(x) e\left(u_{n}\right) G_{z} \geqslant \varepsilon_{0}^{2}\right\},
$$

where $\varepsilon_{0}$ is given by Lemma 2.3. Then the following facts are known: 
FACT 2.4 ([13]). $-\Sigma$ is closed and $\mathcal{P}^{m}\left(\Sigma \cap P_{R}(0)\right) \leqslant C_{R}<\infty$, for any $R<\infty$.

FACT 2.5 ([13]). $-u_{n} \rightarrow u$ strongly in $H_{\mathrm{loc}}^{1} \cap C_{\mathrm{loc}}^{1}\left(M \times R_{+} \backslash \Sigma, R^{k}\right)$.

FACT 2.6 ([13]). $-u \in C^{\infty}\left(M \times R_{+} \backslash \Sigma, N\right)$ is a weak solution of the heat flow of harmonic maps (1.0).

FACT 2.7 ([9]). - For any $t>0$, let $\Sigma_{t}=\Sigma \cap\{t\}$. Then $H^{m-2}\left(\Sigma_{t} \cap K\right) \leqslant C_{K}<\infty$, for any compact $K \subset M$.

FACT 2.8 ([31]). $-\operatorname{sing}(u) \cup \operatorname{spt}(v)=\Sigma$, spt $\eta \subset \Sigma$.

FACT 2.9 ([31]). - For any $z \in M \times R_{+}, \int_{T_{R}(z)} \eta^{2}(x) G_{z}(x, t) \mathrm{d} \mu(x, t)$ is monotonically nondecreasing with respect to $R$. Hence

$$
\Theta^{m}(\mu, z)=\lim _{R \downarrow 0} \int_{T_{R}(z)} \eta^{2}(x) G_{z}(x, t) \mathrm{d} \mu(x, t)
$$

exists for all $x \in M \times R_{+}$and is upper-semicontinuous function of $z$. In particular,

$$
\Sigma=\left\{z \in M \times R_{+}: \varepsilon_{0}^{2} \leqslant \Theta^{m}(\mu, z)<\infty\right\} .
$$

FACT 2.10 ([31]). - For $\mathcal{P}^{m}$ a.e. $z \in \Sigma$,

$$
\Theta^{m}(u, z)=\lim _{R \downarrow 0} R^{-m} \int_{P_{R}(z)}|D u|^{2}(x, t) \mathrm{d} x \mathrm{~d} t=0, \quad \text { and } \quad \Theta^{m}(\nu, z)=\Theta^{m}(\mu, z) .
$$

FACT 2.11 ([31]). $-u_{n}$ doesn't converge to $u$ strongly in $H_{\mathrm{loc}}^{1}\left(M \times R_{+}, R^{k}\right)$ if and only if $\mathcal{P}^{m}(\Sigma)>0$ and there exists at least one harmonic $S^{2}$ in $N$.

Now, we add two more lemmas needed later.

LEMMA 2.12. - Under the same notations as above, we have

$$
\lim _{n \rightarrow \infty} \int_{M \times[t, T]} \frac{1}{\varepsilon_{n}^{2}} F\left(u_{n}\right)(x, t) \mathrm{d} x \mathrm{~d} t=0, \quad \forall 0<t<T<\infty .
$$

Proof. - It follows from the Fact 2.5 that for any $\beta>0$, we have

$$
\lim _{n \rightarrow \infty} \int_{M \times[t, T] \backslash\left(\Sigma_{t}^{T}\right)_{\beta}} \frac{1}{\varepsilon_{n}^{2}} F\left(u_{n}\right)(x, t) \mathrm{d} x \mathrm{~d} t=0
$$

where $\Sigma_{t}^{T}=\bigcup_{s=t}^{T}\left(\Sigma_{s} \times\{s\}\right)$ and $\left(\Sigma_{t}^{T}\right)_{\beta}=\left\{z \in M \times[t, T]: \delta\left(z, \Sigma_{t}^{T}\right) \leqslant \beta\right\}$. It suffices to show that

$$
\lim _{n \rightarrow \infty} \int_{\left(\Sigma_{t}^{T}\right)_{\beta}} \frac{1}{\varepsilon_{n}^{2}} F\left(u_{n}\right)(x, t) \mathrm{d} x \mathrm{~d} t=\mathrm{O}(\beta)
$$


On the other hand, for any $z_{0} \in \Sigma_{t}^{T}$, (2.3) gives

$$
\Psi\left(\mu, z_{0}, \frac{\beta}{2}\right)+\lim _{n \rightarrow \infty} \int_{\frac{\beta}{2}}^{\beta} r^{-1} \int_{T_{r}\left(z_{0}\right)} \frac{1}{\varepsilon_{n}^{2}} F\left(u_{n}\right) G_{z_{0}} \leqslant \mathrm{e}^{C \beta} \Psi\left(\mu, z_{0}, \beta\right)
$$

for sufficiently small $\beta>0$. Moreover, since $\lim _{\beta \downarrow 0} \Psi\left(\mu, z_{0}, \beta\right)$ exists, we may assume that

$$
\left|\Psi\left(\mu, z_{0}, \beta\right)-\Psi\left(\mu, z_{0}, \frac{\beta}{2}\right)\right|=\mathrm{O}(\beta), \quad \forall \beta \ll 1 .
$$

Therefore we have

$$
\int_{\frac{\beta}{2}}^{\beta} r^{-1} \lim _{n \rightarrow \infty} \int_{T_{r}\left(z_{0}\right)} \frac{1}{\varepsilon_{n}^{2}} F\left(u_{n}\right) G_{z_{0}}=\mathrm{O}(\beta) .
$$

This, combined with the Fubini's theorem, implies

$$
\lim _{n \rightarrow \infty} \int_{T_{\bar{\beta}}\left(z_{0}\right)} \frac{1}{\varepsilon_{n}^{2}} F\left(u_{n}\right) G_{z_{0}}=\mathrm{O}(\beta)
$$

for some $\bar{\beta} \in\left(\frac{\beta}{2}, \beta\right)$. In particular, we have

$$
\lim _{n \rightarrow \infty} \int_{B_{\frac{\beta}{2}}\left(x_{0}\right) \times\left[t_{0}-\beta^{2}, t_{0}-\frac{\beta^{2}}{4}\right]} \frac{1}{\varepsilon_{n}^{2}} F\left(u_{n}\right)=\mathrm{O}(\beta) .
$$

This, combined with a simple covering argument, implies (2.7).

LEMMA 2.13. - There exists a subsequence of $n^{\prime} \rightarrow \infty$ such that

$$
e\left(u_{n^{\prime}}\right)(x, t) \mathrm{d} x \rightarrow \mu_{t}, \quad \forall t>0,
$$

as convergences of Radon measures, for a family of nonnegative Radon measures $\left\{\mu_{t}\right\}_{t>0}$ on $M$. In particular, $\mu_{t}=\frac{1}{2}|D u|^{2}(x, t) \mathrm{d} x+v_{t}, \mu=\mu_{t} \mathrm{~d} t$, and $v=v_{t} \mathrm{~d} t$.

Proof. - The idea here is similar to that of Brakke [8] (see also Ilmanen [20]). For completeness, we outline it here. Let $\phi \in C_{0}^{2}\left(M, R_{+}\right)$. Then (2.1) implies

$$
\begin{aligned}
\frac{\mathrm{d}}{\mathrm{d} t} \int_{M} \phi e\left(u_{n}\right) & =-\int_{M} \phi\left|\partial_{t} u_{n}\right|^{2}-\int_{M} D \phi D u_{n} \partial_{t} u_{n} \\
& \leqslant C(\phi) \int_{M} \phi(x)\left|D u_{n}\right|^{2}(x, t) \mathrm{d} x \leqslant C(\phi) E_{0},
\end{aligned}
$$


where $C(\phi)=\sup _{\phi>0} \frac{|D \phi|^{2}}{2 \phi} \leqslant \sup _{\phi>0}\left|D^{2} \phi\right|>0$ and $E_{0}=E\left(u_{n}(\cdot, 0)\right)$ denotes the energy of the initial data. Hence

$$
\int_{M} \phi e\left(u_{n}\right)-C(\phi) E_{0} t
$$

is monotonically nonincreasing with respect to $t>0$. Let $B \subset R_{+}$be a countable dense subset. By the weak compactness of Radon measures with locally bounded masses, and a diagonal process, we can assume that

$$
e\left(u_{n}\right)(x, t) \mathrm{d} x \rightarrow \mu_{t}, \quad \forall t \in B
$$

Now, let $\left\{\phi_{i}\right\}_{i} \geqslant 1$ be a countable dense subset in $C_{0}^{2}\left(M, R_{+}\right)$. By the monotonicity of $\int_{M} \phi e\left(u_{n}\right)-C(\phi) E_{0} t$, there exists a co-countable set $C \subset R_{+}$such that for any $t \in C$ and $i \geqslant 1, \mu_{s}\left(\phi_{i}\right)$ is continuous at $t$ as a function of $s \in B$. For any fixed $t \in C$, there exists a further subsequence $n_{j} \rightarrow \infty$ and a limit $\mu_{t}$ such that $\mu_{n_{j}} \rightarrow \mu_{t}$. Hence $\left\{\mu_{s}\left(\phi_{i}\right)\right\}_{s \in B \cup\{t\}}$ is continuous at $t$, for all $i \geqslant 1$. Hence $\mu_{t}$ is uniquely determined by $\mu_{s}$ for $s \in B$. Therefore, $\mu_{n} \rightarrow \mu_{t}$. Note that $R_{+} \backslash C$ is countable, we can do another diagonal process to show the result on $R_{+}$.

\section{Dimension reduction and stratification of the concentration set}

In this section, we will establish the stratification result for the energy concentration set $\Sigma$. To do it, we consider the space, $T_{z} \mu$, of all tangent measures of $\mu$ at each $z \in \Sigma$. We show that for each $\mu^{0} \in T_{z} \mu, \mu^{0} \mathrm{~L} R_{-}^{m+1}$ is invariant under the parabolic dilation $\mathcal{P}_{\lambda}$ for all $\lambda>0$. For each $\mu^{0} \in T_{z} \mu$, we then associate a nonnegative integer $d$ which is the dimension of the largest $\mathcal{P}_{\lambda}$-invariant subspace inside $M\left(\Theta^{m}\left(\mu^{0}\right)\right)=$ $\left\{z \in R^{m+1}: \Theta^{m}\left(\mu^{0}, z\right)=\Theta^{m}\left(\mu^{0}, 0\right)\right\}$. Using this integer, we can stratify $\Sigma$ accordingly. The proof of the stratification is based on an extension of the well-known Federer's dimension reduction argument [16,3], and [33]. We would also like to remark that a similar scheme has been carried out by White [37] in an abstract way, with applications to mean curvature flows.

For simplicity, we assume $M=R^{m}$ in this section. Note that the norm of the parabolic metric in $R^{m+1}$ is

$$
\|(x, t)\|=\max \{|x|, \sqrt{|t|}\} .
$$

Define the parabolic dilation by

$$
\mathcal{P}_{z_{0}, \lambda}(x, t)=\left(\frac{x-x_{0}}{\lambda}, \frac{t-t_{0}}{\lambda^{2}}\right)
$$

for $z_{0}=\left(x_{0}, t_{0}\right) \in R^{m+1}$ and $\lambda>0$. Define the Euclidean dilation by

$$
\mathcal{D}_{x_{0}, \lambda}(x)=\frac{x-x_{0}}{\lambda}, \quad x \in R^{m}
$$


for $x_{0} \in R^{m}$ and $\lambda>0$. Denote $R_{+}^{m+1}=R^{m} \times R_{+}$and $R_{-}^{m+1}=R^{m} \times R_{-}$. The Hausdorff dimension of a subset $S \in R^{m+1}$ is the Hausdorff dimension with respect to the parabolic metric $\delta$. We write $\Sigma=\left\{\left(\Sigma_{t}, t\right): 0<t<\infty\right\}$, here $\Sigma_{t}=\Sigma \cap\{t\}$. For $\varepsilon_{n}>0$, let $u_{n}: R_{+}^{m+1} \rightarrow R^{k}$ solve (1.3)-(1.4), with $\varepsilon=\varepsilon_{n}$. Recall that (2.4) implies that for $0<$ $R_{2} \leqslant R_{1}<\frac{\sqrt{t_{0}}}{2}$

$$
\begin{aligned}
& \Phi\left(u_{n}, z_{0}, R_{1}\right)-\Phi\left(u_{n}, z_{0}, R_{2}\right) \\
& \quad \geqslant \frac{1}{2} \int_{R_{2}}^{R_{1}} \int_{R^{m}} \frac{\left|2\left(t_{0}-t\right) \partial_{t} u_{n}-\left(x-x_{0}\right) D u_{n}\right|^{2}}{t_{0}-t} G_{z_{0}} \mathrm{~d} z
\end{aligned}
$$

where $\Phi\left(u_{n}, z_{0}, R\right)=R^{2} \int_{t=t_{0}-R^{2}} e\left(u_{n}\right)(x, t) G_{z_{0}}(x, t) \mathrm{d} x$. By (1.6) and Lemma 2.5, we can assume that

$$
e\left(u_{n}\right)(x, t) \mathrm{d} x \mathrm{~d} t \rightarrow \mu \equiv \mu_{t} \mathrm{~d} t
$$

as convergence of Radon measures in $R_{+}^{m+1}$, for some nonnegative Radon measures $\left\{\mu_{t}\right\}_{t>0}$ on $R^{m}$. From (1.6) and (3.1), we have

$$
\sup _{(x, t, r) \in R^{m} \times R_{+} \times R_{+}} r^{-m} \mu\left(P_{r}(x, t)\right)<\infty
$$

and

$$
\Theta^{m}\left(\mu, z_{0}\right)=\lim _{R \downarrow 0} R^{2} \int_{t=t_{0}-R^{2}} G_{z_{0}} \mathrm{~d} \mu_{t_{0}}
$$

exists for all $z_{0} \in R_{+}^{m+1}$. Moreover, the Fact 2.9 implies that

$$
\Sigma=\left\{z \in R_{+}^{m+1}: \varepsilon_{0}^{2} \leqslant \Theta^{m}(\mu, z)<\infty\right\} .
$$

For $z_{0} \in \Sigma$ and $\lambda_{i} \downarrow 0$, we define the parabolic rescalings of $\mu, \mathcal{P}_{z_{0}, \lambda_{i}^{-1}}(\mu)$, by

$$
\mathcal{P}_{z_{0}, \lambda_{i}^{-1}}(\mu)(A)=\lambda_{i}^{-m} \mu\left(\mathcal{P}_{z_{0}, \lambda_{i}^{-1}}(A)\right), \quad \forall \text { Borel } A \subset R^{m+1} .
$$

Then it follows from (3.2) that we can find a subsequence $\lambda_{i^{\prime}}$ of $\lambda_{i}$ and a nonnegative Radon measure $\mu^{0}$ on $R^{m+1}$ such that

$$
\mathcal{P}_{z 0, \lambda_{i^{\prime}}^{-1}} \rightarrow \mu^{0}
$$

as convergence of Radon measures on $R^{m+1}$.

DEFINITION 3.1. - For any $z_{0} \in \Sigma$, the tangent measure cone of $\mu$ at $z_{0}, T_{z_{0}}(\mu)$, consists of all nonnegative Radon measures on $R^{m+1}$ obtained by

$$
T_{z_{0}}(\mu)=\left\{\mu^{0}: \text { there exists a } r_{i} \downarrow 0 \text { such that } \mathcal{P}_{z_{0}, \lambda_{i}^{-1}}(\mu) \rightarrow \mu^{0}\right\} .
$$


Note that for any $z_{0} \in \Sigma$ and $\mu^{0} \in T_{z_{0}}(\mu)$, we have $\mu^{0}=\mu_{s}^{0} \mathrm{~d} s$ and for any $(x, t) \in$ $R^{m+1}$

$$
\Theta^{m}\left(\mu^{0},(x, t), r\right) \equiv r^{2} \int_{s=t-r^{2}} G_{(x, t)}(y, s) \mathrm{d} \mu_{s}^{0}(y)
$$

is monotonically nondecreasing with respect to $r$. Hence

$$
\Theta^{m}\left(\mu^{0},(x, t)\right)=\lim _{r \downarrow 0} \Theta^{m}\left(\mu^{0},(x, t), r\right)
$$

exists for any $(x, t) \in R^{m+1}$ and is upper semicontinuous.

LEMMA 3.2. - For any $z_{0} \in \Sigma$ and $\mu^{0} \in T_{z_{0}}(\mu)$. Then $\mu^{0} \mathrm{~L} R_{-}^{m+1}$ is invariant under all parabolic dilations $\mathcal{P}_{\lambda}$, i.e.,

$$
\mathcal{P}_{\lambda}\left(\mu^{0} \mathrm{~L} R_{-}^{m+1}\right)=\mu^{0} \mathrm{~L} R_{-}^{m+1}
$$

Proof. - It follows from Lemma 2.5 that $\mu^{0}=\mu_{t}^{0} \mathrm{~d} t$. Therefore,

$$
\begin{aligned}
\mathcal{P}_{\lambda}\left(\mu^{0} \mathrm{~L} R_{-}^{m+1}\right) & =\mathcal{P}_{\lambda}\left(\left\{\left(\mu_{t}^{0}, t\right): t \leqslant 0\right\}\right) \\
& =\left\{\mathcal{D}_{\lambda}\left(\left(\mu_{t}^{0}\right), \lambda^{2} t\right): t \leqslant 0\right\} \\
& =\left\{\mathcal{D}_{\lambda}\left(\left(\mu_{\frac{t}{\lambda^{2}}}^{0}\right), t\right): t \leqslant 0\right\} .
\end{aligned}
$$

Here $\mathcal{D}_{\lambda}\left(\mu_{t}^{0}\right)(A)=\lambda^{2-m} \mu_{t}^{0}(\lambda A)$ for any borel set $A \subset R^{m}$. Hence, it suffices to show that

$$
\mathcal{D}_{\lambda}\left(\mu_{\frac{t}{\lambda^{2}}}^{0}\right)=\mu_{t}^{0}, \quad \forall t \leqslant 0 .
$$

Since $\lambda>0$ is arbitrary, it suffices to prove (3.4) for $t=-1$, which is equivalent to

$$
\lambda^{m-2} \int_{R^{m}} \phi(\lambda x) G(\lambda x,-1) \mathrm{d} \mu_{-\lambda^{-2}}^{0}(x)=\int_{R^{m}} \phi(x) G(x,-1) \mathrm{d} \mu_{-1}^{0}(x),
$$

for any $\phi \in C_{0}^{1}\left(R^{m}\right)$ and $G=G_{(0,0)}$. On the other hand, we know that there exists $\lambda_{n} \downarrow 0$ such that $v_{n}(x, t)=u_{n}\left(x_{0}+\lambda_{n} x, t_{0}+\lambda_{n}^{2} t\right)$ satisfies (1.3), with $\varepsilon_{n}$ replaced by $\bar{\varepsilon}_{n}=\frac{\varepsilon_{n}}{\lambda_{n}}$, and

$$
e\left(v_{n}\right)(x, t) \mathrm{d} x \equiv e_{\bar{\varepsilon}_{n}}\left(v_{n}\right)(x, t) \mathrm{d} x \rightarrow \mu_{t}^{0}, \quad \forall t \in R
$$

as convergence of Radon measures on $R^{m}$. Then, for any $R>0$,

$$
\begin{aligned}
R^{2} \int_{t=-R^{2}} G \mathrm{~d} \mu_{t}^{0} & =\lim _{n \rightarrow \infty} R^{2} \int_{t=-R^{2}} e\left(v_{n}\right)(x, t) G(x, t) \mathrm{d} x \\
& =\lim _{\lambda_{n} \downarrow 0}\left(R \lambda_{n}\right)^{2} \int_{t=t_{0}-R^{2} \lambda_{n}^{2}} G_{z 0}(x, t) \mathrm{d} \mu_{t} \\
& =\Theta^{m}\left(\mu, z_{0}\right) .
\end{aligned}
$$


This, combined with (3.1), implies that, for any $0<r_{1}<r_{2}<\infty$,

$$
\lim _{n \rightarrow \infty} \int_{t=-r_{1}^{2}}^{t=-r_{2}^{2}} \int_{R^{m}}\left|x D v_{n}+2 t \partial_{t} v_{n}\right|^{2} G=0
$$

Therefore, in oder to prove (3.5), it suffices to show

$$
\lim _{n \rightarrow \infty} \frac{\mathrm{d}}{\mathrm{d} \lambda}\left(\lambda^{m-2} \int_{t=-1} \phi(\lambda x) G(\lambda x,-1) e\left(v_{n}\right)\left(x,-\lambda^{-2}\right) \mathrm{d} x\right)=0 .
$$

Note that

$$
\begin{aligned}
\frac{\mathrm{d}}{\mathrm{d} \lambda} & \left(\lambda^{m-2} \int_{t=-1} \phi(\lambda x) G(\lambda, x,-1) e\left(v_{n}\right)\left(x,-\lambda^{-2}\right)\right) \\
& =\frac{\mathrm{d}}{\mathrm{d} \lambda}\left(\int_{t=-1} \phi(x) G(x, t) e\left(v_{n}^{\lambda}\right)(x, t) \mathrm{d} x\right) \\
& =-\int_{t=-1} G D \phi D v_{n}^{\lambda} \frac{\mathrm{d}}{\mathrm{d} \lambda} v_{n}^{\lambda}-\int_{t=-1} \phi\left(\partial_{t} v_{n}^{\lambda}-\frac{1}{2} x D v_{n}^{\lambda}\right) \frac{\mathrm{d}}{\mathrm{d} \lambda} v_{n}^{\lambda} G \\
& =-\frac{1}{2 \lambda} \int_{t=-\lambda^{2}}\left|y D v_{n}+2 t \partial_{t} v_{n}\right|^{2} G \phi\left(\frac{y}{\lambda}\right)-\int_{t=-\lambda^{2}} D \phi\left(\frac{y}{\lambda}\right) G D v_{n}\left(y D v_{n}+2 t \partial_{t} v_{n}\right) .
\end{aligned}
$$

Here $v_{n}^{\lambda}(x, t)=v_{n}\left(\lambda x, \lambda^{2} t\right)$ and $e\left(v_{n}^{\lambda}\right)(x, t)=e_{\frac{\bar{\varepsilon}_{n}}{\lambda}}\left(v_{n}^{\lambda}\right)(x, t)$. Hence, integrating the identity from 1 to $\lambda$ and using (3.6), we see that (3.5) holds.

From Lemma 3.2, we see that for any $z_{0} \in \Sigma$ and $\mu^{0} \in T_{z_{0}} \mu$,

$$
R^{2} \int_{t=-R^{2}} G \mathrm{~d} \mu_{t}^{0}=\Theta^{m}\left(\mu^{0}, 0\right)=\Theta^{m}\left(\mu, z_{0}\right), \quad \forall R>0
$$

and, for any $z \in R_{-}^{m+1}$ and $\lambda>0$,

$$
\Theta^{m}\left(\mu^{0}, z\right)=\Theta^{m}\left(\mu_{0}, \mathcal{P}_{\lambda}(z)\right)
$$

In general, we have

LEMMA 3.3. - For $z_{0} \in \Sigma$ and $\mu^{0} \in T_{z_{0}}(\mu)$, we have

(1) $\Theta^{m}\left(\mu^{0}, z\right) \leqslant \Theta^{m}\left(\mu^{0}, 0\right), \forall z \in R^{m+1}$.

(2) If $z \in R^{m+1}$ satisfies $\Theta^{m}\left(\mu^{0}, z\right)=\Theta^{m}\left(\mu^{0}, 0\right)$, then

$$
\Theta^{m}\left(\mu^{0}, z+v\right)=\Theta^{m}\left(\mu^{0}, z+\mathcal{P}_{\lambda} v\right), \quad \forall \lambda>0, v \in R_{-}^{m+1} .
$$

Proof. $-(1)$ For $\mu^{0} \in T_{z_{0}}(\mu)$, there exists $r_{i} \downarrow 0$ such that $\mathcal{P}_{z_{0}, r_{i}}(\mu) \rightarrow \mu^{0}$. For any $r>0$ and $z=(x, t) \in R^{m+1}$, we have 


$$
\begin{aligned}
\Theta^{m}\left(\mu^{0}, z\right) & \leqslant \Theta^{m}\left(\mu^{0}, z, r\right) \\
& =\lim _{r_{i} \downarrow 0} \Theta^{m}\left(\mathcal{P}_{z_{0}, r_{i}}(\mu), z, r\right) \\
& =\lim _{r_{i} \downarrow 0} \Theta^{m}\left(\mu, z_{0}+\left(r_{i} x, r_{i}^{2} t\right), r_{i}^{2} r\right) \\
& \leqslant \Theta^{m}\left(\mu, z_{0}\right)=\Theta^{m}\left(\mu^{0}, 0\right) .
\end{aligned}
$$

Here we have used the upper semicontinuity of $\Theta^{m}(\mu, \cdot, \cdot)$ in its last two variables.

(2) From the proof of (1), we see that if $\Theta^{m}\left(\mu^{0}, z\right)=\Theta^{m}\left(\mu^{0}, 0\right)$ then the inequalities are all equalities. In particular, $\Theta^{m}\left(\mu^{0}, z, r\right)$ is constant with respect to $r>0$. Applying the argument of Lemma 3.2, we see that $\Theta^{m}\left(\mu^{0}, z+v\right)=\Theta^{m}\left(\mu^{0}, z+\mathcal{P}_{\lambda}(v)\right)$ for any $v \in R_{-}^{m+1}$ and $\lambda>0$.

For any $z_{0} \in \Sigma$ and $\mu^{0} \in T_{z_{0}}(\mu)$, denote

$$
\begin{gathered}
M\left(\Theta^{m}\left(\mu^{0}, \cdot\right)\right) \equiv\left\{z \in R^{m+1}: \Theta^{m}\left(\mu^{0}, z\right)=\Theta^{m}\left(\mu^{0}, 0\right)\right\}, \\
V\left(\Theta^{m}\left(\mu^{0}, \cdot\right)\right) \equiv M\left(\Theta^{m}\left(\mu^{0}, \cdot\right)\right) \cap\{t=0\},
\end{gathered}
$$

and

$$
W\left(\Theta^{m}\left(\mu^{0}, \cdot\right)\right) \equiv\left\{x \in R^{m}: \Theta^{m}\left(\mu^{0},(y, s)\right)=\Theta^{m}\left(\mu^{0},(x+y, s)\right), \forall(y, s) \in R_{-}^{m+1}\right\} .
$$

Then we have

Proposition 3.4. - For $z_{0} \in \Sigma$ and $\mu^{0} \in T_{z_{0}}(\mu)$, we have $V\left(\Theta^{m}\left(\mu^{0}, \cdot\right)\right)=$ $W\left(\Theta^{m}\left(\mu^{0}, \cdot\right)\right)$. In particular, both $V\left(\Theta^{m}\left(\mu^{0}, \cdot\right)\right)$ and $W\left(\Theta^{m}\left(\mu^{0}, \cdot\right)\right)$ are subspaces of $R^{m}$. Moreover, $M\left(\Theta^{m}\left(\mu^{0}, \cdot\right)\right)$ is $V\left(\Theta^{m}\left(\mu^{0}, \cdot\right)\right)$ or $V\left(\Theta^{m}\left(\mu^{0}, \cdot\right)\right) \times(-\infty, a]$ for some $0 \leqslant a \leqslant \infty$ and $\Theta^{m}\left(\mu^{0}, \cdot\right)$ is time-independent up to $t=a$.

Proof. - It is clear that $W\left(\Theta^{m}\left(\mu^{0}, \cdot\right)\right) \subset V\left(\Theta^{m}\left(\mu^{0}, \cdot\right)\right), V\left(\Theta^{m}\left(\mu^{0}, \cdot\right)\right)$ is closed under scalar multiplication, and $n W\left(\Theta^{m}\left(\mu^{0}, \cdot\right)\right) \subset W\left(\Theta^{m}\left(\mu^{0}, \cdot\right)\right)$ for any integer $n$. For any $(x, 0) \neq(0,0) \in V\left(\Theta^{m}\left(\mu^{0}, \cdot\right)\right), v \in R_{-}^{m+1}$, and $\lambda>0$, we have

$$
\begin{aligned}
\Theta^{m}\left(\mu^{0},(x, 0)+v\right) & =\Theta^{m}\left(\mu^{0},(x, 0)+\mathcal{P}_{\lambda} v\right) \\
& =\Theta^{m}\left(\mu^{0}, \mathcal{P}_{\lambda^{-1}}\left((x, 0)+\mathcal{P}_{\lambda} v\right)\right) \\
& =\Theta^{m}\left(\mu^{0}, \mathcal{P}_{\lambda^{-1}}(x, 0)+v\right)
\end{aligned}
$$

so that

$$
\Theta^{m}\left(\mu^{0},(x, 0)+v\right)=\Theta^{m}\left(\mu^{0}, \mathcal{P}_{\lambda^{-1}}(x, 0)+v\right) .
$$

Note that $v-\mathcal{P}_{\lambda^{-1}}(x, 0) \in R_{-}^{m+1}$. Hence, replacing $v$ by $v-\mathcal{P}_{\lambda^{-1}}(x, 0)$ gives

$$
\Theta^{m}\left(\mu^{0},(x, 0)+v-\mathcal{P}_{\lambda^{-1}}(x, 0)\right)=\Theta^{m}\left(\mu^{0}, v\right) .
$$

Taking $\lambda$ into zero and using the upper semicontinuity of $\Theta^{m}\left(\mu^{0}, \cdot\right)$, we obtain, from (3.11) and (3.12),

$$
\Theta^{m}\left(\mu^{0}, v\right)=\Theta^{m}\left(\mu^{0},(x, 0)+v\right), \quad \forall v \in R_{-}^{m+1} .
$$


This implies that $V\left(\Theta^{m}\left(\mu^{0}, \cdot\right)\right) \subset W\left(\Theta^{m}\left(\mu^{0}, \cdot\right)\right)$. Hence $V\left(\Theta^{m}\left(\mu^{0}, \cdot\right)\right)=W\left(\Theta^{m}\left(\mu^{0}, \cdot\right)\right)$ and is a subspace of $R^{m}$.

Suppose that $X=(x, t) \in M\left(\Theta^{m}\left(\mu^{0}, \cdot\right)\right)$, with $t<0$. Then, for any $Y=(y, s)$ with $s \leqslant t$ and $\lambda>0$, we have

$$
\Theta^{m}\left(\mu^{0}, \mathcal{P}_{\lambda^{-1}}(Y)\right)=\Theta^{m}\left(\mu^{0}, Y\right)=\Theta^{m}\left(\mu^{0}, X+\mathcal{P}_{\lambda^{-1}}(Y-X)\right) .
$$

Note that $s \lambda^{-2} \leqslant s \leqslant t$, for $\lambda<1$. Hence replacing $Y$ by $\mathcal{P}_{\lambda}(Y)$, we get

$$
\Theta^{m}\left(\mu^{0}, Y\right)=\Theta^{m}\left(\mu^{0}, X+Y-\mathcal{P}_{\lambda^{-1}}(X)\right) .
$$

Taking $\lambda$ into zero, we have $\Theta^{m}\left(\mu^{0}, Y\right) \leqslant \Theta^{m}\left(\mu^{0}, X+Y\right)$. By substituting $Y$ by $Y+\mathcal{P}_{\lambda^{-1}}(X)$, we also get

$$
\Theta^{m}\left(\mu^{0}, Y+\mathcal{P}_{\lambda^{-1}}(X)\right)=\Theta^{m}\left(\mu^{0}, X+Y\right)
$$

this implies $\Theta^{m}\left(\mu^{0}, Y\right) \geqslant \Theta^{m}\left(\mu^{0}, X+Y\right)$. Therefore, we have

$$
\Theta^{m}\left(\mu^{0}, Y\right)=\Theta^{m}\left(\mu^{0}, X+Y\right), \quad \forall X=(x, t), Y=(y, s), s \leqslant t<0 .
$$

This implies, by choosing $Y=(n-1) X$,

$$
\begin{aligned}
\Theta^{m}\left(\mu^{0}, 0\right) & =\Theta^{m}\left(\mu^{0},(n x, n t)\right)=\Theta^{m}\left(\mu^{0}, \mathcal{P}_{n}(n x, n t)\right) \\
& =\Theta^{m}\left(\mu^{0},\left(x, \frac{t}{n}\right)\right) \leqslant \Theta^{m}\left(\mu^{0},(x, 0)\right) .
\end{aligned}
$$

Therefore, $(x, 0) \in V\left(\Theta^{m}\left(\mu^{0}, \cdot\right)\right)=W\left(\Theta^{m}\left(\mu^{0}, \cdot\right)\right)$ and $(0, t) \in M\left(\Theta^{m}\left(\mu^{0}, \cdot\right)\right)$. In particular, $\Theta^{m}\left(\mu^{0}, \cdot\right)$ is time-independent up to time $t=0$.

If $X=(x, t) \in V\left(\Theta^{m}\left(\mu^{0}, \cdot\right)\right)$, with $t>0$, then we can prove similarly that $\Theta^{m}\left(\mu^{0}, \cdot\right)$ is time-independent up to time $t$. Let $t=a \geqslant 0$ be the maximal number such that $\Theta^{m}\left(\mu^{0}, \cdot\right)$ is time-independent up to $t=a$. Then one gets $M\left(\Theta^{m}\left(\mu^{0}, \cdot\right)\right)=V\left(\Theta^{m}\left(\mu^{0}, \cdot\right)\right) \times$ $(-\infty, a]$.

Definition 3.5. - For $z_{0} \in \Sigma$ and $\mu^{0} \in T_{z_{0}}(\Sigma)$, define $\operatorname{dim}\left(\Theta^{m}\left(\mu^{0}, \cdot\right)\right)$ by $\operatorname{dim}\left(\Theta^{m}\left(\mu^{0}, \cdot\right)\right)= \begin{cases}\operatorname{dim}\left(V\left(\Theta^{m}\left(\mu^{0}, \cdot\right)\right)\right)+2, & \text { if } M\left(\Theta^{m}\left(\mu^{0}, \cdot\right)\right)=V\left(\Theta^{m}\left(\mu^{0}, \cdot\right)\right) \times R \\ \operatorname{dim}\left(V\left(\Theta^{m}\left(\mu^{0}, \cdot\right)\right)\right), & \text { otherwise. }\end{cases}$

Now we are ready to prove the main theorem of this section.

THEOREM 3.6. - For $0 \leqslant k \leqslant m$, let

$$
\Sigma_{k}=\left\{z_{0} \in \Sigma: \operatorname{dim}\left(\Theta^{m}\left(\mu^{0}, \cdot\right)\right) \leqslant k, \forall \mu^{0} \in T_{z_{0}}(\mu)\right\} .
$$

Then $\operatorname{dim}\left(\Sigma_{k}\right) \leqslant k$ for $0 \leqslant k \leqslant m$, and $\Sigma_{0}$ is discrete. In particular, $\Sigma=\Sigma_{0} \cup\left(\Sigma_{1} \backslash\right.$ $\left.\Sigma_{0}\right) \cup \cdots \cup\left(\Sigma_{m} \backslash \Sigma_{m-1}\right)$, and for $\mathcal{P}^{m}$ a.e. $z \in \Sigma$, there exists at least one $\mu^{0} \in T_{z}(\mu)$ such that

$$
\mu^{0}=\Theta^{m}(\mu, z)\left(H^{m-2} \mathrm{~L} T_{m-2}\right) \times\left(L^{1} \mathrm{~L} R\right) .
$$

Here $T_{m-2} \subset R^{m}$ is a $(m-2)$-plane. 
Proof of Theorem 3.6. - This is essentially an extension of the Federer's dimension reduction argument. It suffices to show that if $\mathcal{P}^{d}\left(\Sigma_{k}\right)>0$ then $d \leqslant k$. Thus we only consider such a $d$. First, denote $A_{z, r}=\mathcal{P}_{z, r}(A)$ for $A \subset R^{m+1}, z \in R^{m+1}$, and $\lambda>0$. Let

$$
\begin{aligned}
\mathcal{C}= & \left\{V \times R, \text { or } V: V \subset R^{m} \text { a subspace of } \operatorname{dim}(V) \leqslant k-2\right\} \\
& \cup\left\{V \times R_{-}: V \subset R^{m} \text { a subspace of } \operatorname{dim}(V) \leqslant k\right\} .
\end{aligned}
$$

Then we have

Claim 3.7. - For any $z_{0} \in \Sigma_{k}$ and $r>0$ there exists $\eta=\eta(z, \varepsilon)>0$ such that for any $\rho \in(0, \varepsilon)$

$$
\left(\left\{w \in P_{\rho}(z): \Theta^{m}(\mu, w) \geqslant \Theta^{m}(\mu, z)-\eta\right\}\right)_{z, \rho} \subset \varepsilon \text {-neighborhood of } C,
$$

for some $C \in \mathcal{C}$. Here $\varepsilon$-neighborhood is measured with respect to the parabolic metric $\delta$.

For, otherwise, there exist $\varepsilon_{0}>0, z_{0} \in \Sigma_{k}$, and $\rho_{i}, \eta_{i} \downarrow 0$ such that

$$
\begin{aligned}
B_{i} \equiv & \left\{z \in P_{1}(0): \Theta^{m}\left(\mathcal{P}_{z_{0}, \rho_{i}}(\mu), z\right) \geqslant \Theta^{m}\left(\mu, z_{0}\right)-\eta_{i}\right\} \\
& \not \subset \varepsilon_{0} \text {-neighborhood of any } C \in \mathcal{C} .
\end{aligned}
$$

On the other hand, we may assume that $\mathcal{P}_{z_{0}, \rho_{i}}(\mu) \rightarrow \mu^{0} \in T_{z_{0}}(\mu)$ and $B_{i} \rightarrow B \subset$ $M\left(\Theta^{m}\left(\mu^{0}, \cdot\right)\right)=\left\{z \in R^{m+1}: \Theta^{m}\left(\mu^{0}, z\right)=\Theta^{m}\left(\mu, z_{0}\right)\right\}$. By Lemma 3.4, we know that, among the four possibilities of $M\left(\Theta^{m}\left(\mu^{0}, \cdot\right)\right)$, only $M\left(\Theta^{m}\left(\mu^{0}, \cdot\right)=V\left(\Theta^{m}\left(\mu^{0}, \cdot\right)\right) \times\right.$ $(-\infty, a]$ for some $a>0$ is not in $\mathcal{C}$. However, even for such a possibility, we can find $r_{i} \downarrow 0$ such that $\mathcal{P}_{r_{i}}\left(\mu^{0}\right) \rightarrow \mu^{1}$, and by the uppersemicontinuity

$$
\Theta^{m}\left(\mu^{1}, w\right)=\Theta^{m}\left(\mu^{1}, 0\right)=\Theta^{m}\left(\mu^{0}, 0\right), \quad \forall w \in V\left(\Theta^{m}\left(\mu^{0}, \cdot\right)\right) \times R
$$

this implies that $M\left(\Theta^{m}\left(\mu^{0}, \cdot\right)\right) \subset M\left(\Theta^{m}\left(\mu^{1}, \cdot\right)\right) \in \mathcal{C}$. Therefore we get the desired contradiction.

Now we proceed as follows. Let $\varepsilon_{i} \downarrow 0$ and decompose $\Sigma_{k}=\bigcup_{i, q \geqslant 1} \Sigma_{k, i, q}$, here $\Sigma_{k, i, q}$ denotes points where $\Theta^{m}(\mu, \cdot) \in\left((q-1) \varepsilon_{i}, q \varepsilon_{i}\right)$ and Claim 3.7 holds with $\varepsilon=\varepsilon_{i}$. Therefore, for each $i$, there exists $q_{i} \geqslant 1$ such that $\mathcal{P}^{d}\left(\Sigma_{k, i, q_{i}}\right)>0$. By the lower bound for the upper density (cf. [16]), we know that there exist $z_{i} \in \Sigma_{k, i, q_{i}}$ and $r_{i} \downarrow 0$ such that

$$
\mathcal{P}^{d, \infty}\left(\left(\Sigma_{k, i, q_{i}}\right)_{z_{i}, r_{i}}\right) \geqslant 10^{-d} .
$$

Here $\mathcal{P}^{d, \infty}$ denotes the $d$-dimensional Hausdorff measure with size $\infty$. Moreover, for each $z \in\left(\Sigma_{k, i, q_{i}}\right)_{z_{i}, r_{i}}$ there exists $C_{z} \in \mathcal{C}$ such that

$$
\left((\Sigma)_{k, i, q_{i}}\right)_{z_{i}, r_{i}}-z \subset \varepsilon_{i} \text {-neighborhood of } C_{z} .
$$

We may assume that $\left(\Sigma_{k, i, q_{i}}\right)_{z_{i}, r_{i}} \rightarrow \Sigma_{k}^{\infty}$. Then we have

$$
\Sigma_{k}^{\infty}-z \subset C_{z}, \quad \forall z \in \Sigma_{k}^{\infty}, \quad \text { and } \quad \mathcal{P}^{d, \infty}\left(\Sigma_{k}^{\infty}\right) \geqslant 10^{-d} .
$$

For any $C \in \mathcal{C}$, let $\Sigma_{k, j, C}^{\infty}=\left\{z \in \Sigma_{k}^{\infty}: \delta\left(C_{z}, C\right) \leqslant j^{-1}\right\}$. Then for each $j$ there exists $C_{j} \in$ $\mathcal{C}$ such that $\Sigma_{k, j}^{\infty} \equiv \Sigma_{k, j, C_{j}}^{\infty}$ has positive $\mathcal{P}^{d}$-measure. Therefore, there exist $z_{j} \in \Sigma_{k, j}^{\infty}$ and 
$\rho_{j} \downarrow 0$ such that

$$
\mathcal{P}^{d, \infty}\left(\left(\Sigma_{k, j}^{\infty}\right)_{z_{j}, \rho_{j}}\right) \geqslant 10^{-d} .
$$

Assume that $C_{j} \rightarrow C_{\infty} \in \mathcal{C}$ and $\left(\Sigma_{k, j}^{\infty}\right)_{z_{j}, \rho_{j}} \rightarrow \Sigma^{\infty}$. Then $\Sigma^{\infty} \subset C_{\infty}, \mathcal{P}^{d}\left(\Sigma^{\infty}\right)>0$, and $\Sigma^{\infty}-z \subset C_{\infty}$ whenever $z \in \Sigma^{\infty}$. In particular, we have $\Sigma^{\infty} \subset C_{\infty} \cap\left(-C_{\infty}\right)$. But we note that if $C_{\infty}=V_{\infty} \times R_{-}$for some vector subspace $V_{\infty} \subset R^{m}$ then $C_{\infty} \cap\left(-C_{\infty}\right)=$ $V_{\infty}$. Hence $\mathcal{P}^{d}\left(V_{\infty}\right)>0$ implies that $H^{d}\left(V_{\infty}\right)>0$ so that $d \leqslant k$. For $C_{\infty}=V_{\infty}$ or $V_{\infty} \times R$, we see $C_{\infty} \cap\left(-C_{\infty}\right)=C_{\infty}$ so that $\mathcal{P}^{d}\left(\Sigma^{\infty}\right)>0$ implies that $\mathcal{P}^{d}\left(C_{\infty}\right)>0$ so that $d \leqslant k$ again.

\section{Generalized varifolds and rectifiablity of the concentration set}

In this section, we first recall some of the classical theory of varifolds, which was studied by Almgren [3,4] and Allard [6] (see also Simon [33] for details), and at the same time we also recall the notion of generalized varifolds, which was remarked by Almgren [4] and recently adopted by Ambrosio and Soner [1] in their study of the dynamics of Ginzburg-Landau equations with complex values, and Lin [24] in the study of mapping problems.

For simplicity, we assume, throughout this section, that $M=R^{m}$. For $1 \leqslant l \leqslant m$, let $G_{l}(m)$ denote the standard Grassmann manifold of $l$-dimensional unoriented planes in $R^{m}$. For a bounded domain $\Omega \subset R^{m}$, recall a $l$-varifold in $\Omega$ is just a Radon measure in $\Omega \times G_{l}(m)$, and let $V_{l}(\Omega)$ denote all $l$-varifolds in $\Omega$. The weight $\|V\|$ of $V \in V_{l}(\Omega)$ is $\pi_{\#}(V)$, where $\pi(x, A)=x: \Omega \times G_{l}(m) \rightarrow \Omega$. A set $E \subset R^{m}$ is called $l$-rectifiable if except a zero $H^{l}$ measure subset $E$ can be covered by countably many $l$-dimensional $C^{1}$ submanifolds of $R^{m}$. A $V \in V_{l}(\Omega)$ is said to be $l$-rectifiable varifold if there exist a $l$-rectifiable set $E \subset \Omega$ and a locally $H^{l}$ integrable and a positive function $\theta$ such that $V=\delta_{T_{x} E} \theta H^{l} \mathrm{~L} E$ for $H^{l}$ a.e. in $\Omega$, here $T_{x} E$ denotes the tangent plane of $E$ at $x$ and $\delta_{T_{x} E}$ denotes the Dirac mass at $T_{x} E$. Let $R V_{l}(\Omega)$ denote all $l$-rectifiable varifolds.

Now, we recall the definition of generalized varifolds from [1].

DEFINITION 4.1. - A l-dimensional generalized varifold $V$ is a nonnegative Radon measure on $\Omega \times A_{l, m}$, where

$$
A_{l, m}=\left\{A \in R^{m \times m}: A \text { is symmetric, } \operatorname{trace}(A)=l,-l I_{m} \leqslant A \leqslant I_{m}\right\},
$$

where $I_{m}$ denotes the identity matrix of order $m$. The class of all generalized $l$-varifolds is denoted by $V_{l}^{*}(\Omega)$. Again, let $\|V\|$ denote the weight of $v \in V_{l}^{*}(\Omega)$. Since $G_{l}(m) \subset A_{l, m}$, we know that $V_{l}(\Omega) \subset V_{l}^{*}(\Omega)$.

DeFINITION 4.2. - For any given $V \in V_{l}^{*}(\Omega)$, the first variation of $V, \delta V$, is a distribution on $C_{0}^{1}\left(\Omega, R^{m}\right)$ defined by

$$
\delta V(X)=-\int_{\Omega \times A_{l, m}} D X(x): A \mathrm{~d} V(x, A), \quad \forall X \in C_{0}^{1}\left(\Omega, R^{m}\right) .
$$

Here $A: B=\sum_{i j} A_{i j} B_{i j}$ for $A, B \in R^{m \times m} . V$ is called stationary if $\delta V=0$. 
Note that if $\delta V$ is a Radon measure, that is, if

$$
\begin{aligned}
\|\delta V\|(G) & =\sup \left\{|\delta V(X)|: X \in C_{0}^{1}\left(\Omega, R^{m}\right),\|X\|_{L^{\infty}} \leqslant 1, \operatorname{spt}(X) \subset G\right\} \\
& \leqslant C(G)<\infty, \quad \forall G \Subset \Omega .
\end{aligned}
$$

Then the Riesz Representation Theorem implies that

$$
\delta V(X)=\int_{\Omega} X(x) \beta(x) \mathrm{d}\|\delta V\|(x), \quad \forall X \in C_{0}^{1}\left(\Omega, R^{m}\right)
$$

here $\beta$ is a $\|\delta V\|$-measurable, $S^{m-1}$-valued function. If $\|\delta V\| \ll\|V\|$, then we have

$$
\delta V(X)=\int_{\Omega}\langle H(x), X(x)\rangle \mathrm{d}\|V\|(x), \quad \forall X \in C_{0}^{1}\left(\Omega, R^{m}\right),
$$

where $H: \Omega \rightarrow R^{m}$ is a $\|V\|$-measurable function, which we call the generalized mean curvature of $V$.

Note also that the convergence of $V \in V_{l}^{*}(\Omega)$ is understood as weak convergence of Radon measures on $\Omega \times A_{l, m}$. Moreover, if $V_{n} \rightarrow V$, then $\delta V_{n} \rightarrow \delta V$ as distributions. In particular, if $\sup _{n}\left\|\delta V_{n}\right\|(A)<\infty$, then

$$
\|\delta V\|(A) \leqslant \liminf _{n \rightarrow \infty}\left\|\delta V_{n}\right\|(A)<\infty, \quad \text { for } A \subset \Omega .
$$

To motivate the application of generalized varifolds to our problem, we give two examples.

Example 4.3. - For $u \in H^{1}\left(\Omega, R^{k}\right)$, we define $V_{u}(x)=\frac{1}{2} \delta_{A(u)(x)}|D u|^{2}(x) \mathrm{d} x$, where

$$
A(u)(x)= \begin{cases}I_{m}-2 \frac{D u \otimes D u}{|D u|^{2}}(x), & \text { if }|D u|(x) \neq 0 \\ I_{m-2}, & \text { if }|D u|(x)=0 .\end{cases}
$$

Then it is clear that $A(u)(x) \in A_{m-2, m}$. Hence $V_{u} \in V_{m-2}^{*}(\Omega)$. In fact, for any Borel set $B \subset \Omega \times A_{m-2, m}$,

$$
V_{u}(B)=\frac{1}{2} \int_{\pi(B)}|D u|^{2}(x) \mathrm{d} x
$$

where $\pi(B)=\left\{x \in \Omega\right.$ : $\left.\left(x, A_{u}(x)\right) \in B\right\}$. It is clear that $\delta V_{u}$ is given by

$$
\begin{aligned}
\delta V_{u}(X) & =-\int_{\Omega} D X(x): A \mathrm{~d} V_{u}(x, A) \\
& =-\frac{1}{2} \int_{\Omega} D X(x): A(u)(x)|D u|^{2}(x) \mathrm{d} x \\
& =-\frac{1}{2} \int_{\Omega}\left(|D u|^{2}(x) \operatorname{div}(X)-2 D_{i} u D_{j} u X_{i}^{j}\right) \mathrm{d} x .
\end{aligned}
$$


In particular, if $u \in H^{1}(\Omega, N)$ is a stationary harmonic map (see, Bethuel [7] and [23] for the definition and discussion), then we have $\delta V_{u}=0$ so that $V_{u}$ is a stationary generalized $(m-2)$-varifold in $\Omega$.

Example 4.4. - For $\varepsilon_{n} \downarrow 0$, let $u_{n} \in H^{1}\left(\Omega, R^{k}\right)$ be critical points of $I_{\varepsilon_{n}}(\cdot)$, namely, $u_{n}$ satisfies

$$
\Delta u_{n}+\frac{1}{\varepsilon_{n}^{2}} f\left(u_{n}\right)=0, \quad \text { in } \Omega .
$$

Then it follows from [31] that

$$
\delta V_{u_{\varepsilon_{n}}}(X)=-\int_{\Omega} \operatorname{div} X \frac{F\left(u_{n}\right)}{\varepsilon_{n}^{2}}, \quad \forall X \in C_{0}^{1}\left(\Omega, R^{m}\right) .
$$

In particular, if $\sup _{n} I_{\varepsilon_{n}}\left(u_{n}\right)<\infty$, then we may assume that $u_{n} \rightarrow u$ weakly in $H^{1}\left(\Omega, R^{k}\right)$ and

$$
e\left(u_{n}\right)(x) \mathrm{d} x \rightarrow \frac{1}{2}|D u|^{2}(x) \mathrm{d} x+v
$$

as convergence of Radon measures in $\Omega$ for some nonnegative Radon measure $v$ on $\Omega$, and $V_{u_{n}}$ weakly converges to a $V \in V_{m-2}^{*}(\Omega)$. Moreover, it follows from Lemma 2.4 that

$$
\lim _{n \rightarrow \infty} \int_{\Omega} \frac{F\left(u_{n}\right)}{\varepsilon_{n}^{2}}=0 .
$$

Hence $\delta V_{u_{n}}(X) \rightarrow 0$ for any $X \in C_{0}^{1}\left(\Omega, R^{m}\right)$ so that $\delta V=0$ and $V$ is stationary. In Corollary 4.10 below, we show that $v$ is a $(m-2)$-rectifiable Radon measure and $V=$ $V_{u}+V_{R}$, where $V_{R}$ is the $(m-2)$-rectifiable varifold, given by $V_{R}=\delta_{T_{x} \Sigma} \theta H^{m-2} \mathrm{~L} \Sigma$, here $\Sigma$ is a closed $(m-2)$-rectifiable set, and $v=\theta H^{m-2} \mathrm{~L} \Sigma$.

Now we start to discuss the generalized varifold flow, associated with solutions to (1.3)-(1.4). For any $\varepsilon_{n} \downarrow 0$, let $u_{n} \in C^{\infty}\left(\Omega \times R_{+}, R^{k}\right)$ solve (1.3) and satisfy (see also (1.6) and Lemma 2.1):

$$
\sup _{n} \sup _{0<t<\infty}\left(\int_{0}^{t} \int_{\Omega}\left|\partial_{t} u_{n}\right|^{2}+\int_{M} e\left(u_{n}\right)(x, t) \mathrm{d} x\right) \leqslant C<\infty .
$$

For any such a $u_{n}$, we define a generalized $(m-2)$-varifold $V_{n} \in V_{m-2}^{*}\left(\Omega \times R_{+}\right)$as follows

$$
V_{n}(x, t, A)=\delta_{A\left(u_{n}\right)(x, t)}(A) \mu_{t}^{n} \mathrm{~d} t, \quad \forall(x, t, A) \in \Omega \times R_{+} \times A_{m-2, m},
$$

where $A\left(u_{n}\right)$ is defined by (4.6), and $\mu_{t}^{n}(x)=e\left(u_{n}\right)(x, t) \mathrm{d} x$. Let $\pi_{(x, t)}: \Omega \times R_{+} \times$ $A_{m-2, m} \rightarrow \Omega \times R_{+}$be the projection map at $(x, t)$. Then we know that the weight $\left\|V_{n}\right\|=\pi_{(x, t) \#}\left(V_{n}\right)=\mu_{t}^{n} \mathrm{~d} t$. In particular, $\sup _{n}\left\|V_{n}\right\|(G)<\infty$ for any compact subset 
$G \subset \Omega \times R_{+}$. Therefore, we may assume that there exists a generalized $(m-2)$-varifold $V \in V_{m-2}^{*}\left(\Omega \times R_{+}\right)$such that

$$
V_{n} \rightarrow V, \quad\left\|V_{n}\right\|=\mu_{t}^{n} \mathrm{~d} t \rightarrow\|V\|
$$

as convergence of Radon measures. Moreover, by Lemma 2.5, we know that $\|V\|=$ $\mu_{t} \mathrm{~d} t$. We can also represent $V=V_{x, t}\|V\|=V_{x, t} \mu_{t} \mathrm{~d} t$, where for each $(x, t) \in \Omega \times R_{+}$ $V_{x, t}$ is a probability measure on $A_{m-2, m}$. Note that for any compact subset $G \in \Omega \times R_{+}$, $L^{1}$ norm of $-D u_{n} \partial_{t} u_{n}$ on $G$ is uniformly bounded. Hence we may assume that

$$
-D u_{n} \partial_{t} u_{n} \mathrm{~d} x \mathrm{~d} t \rightarrow \sigma
$$

as convergence of Radon measures on $\Omega \times R_{+}$, for some (signed) Radon measures $\sigma$ on $\Omega \times R_{+}$. Since $-D u_{n} \partial_{t} u_{n} \mathrm{~d} x \mathrm{~d} t \ll e\left(u_{n}\right)(x, t) \mathrm{d} x \mathrm{~d} t$, we have

$$
\sigma \ll \mu_{t} \mathrm{~d} t=\|V\| .
$$

By the Riesz Representation Theorem, we know that there exists a $H_{t}(x) \in L_{\|V\|}^{1}(\Omega \times$, $R^{m}$ ) such that

$$
\sigma(x, t)=H_{t}(x) \mu_{t}(x) \mathrm{d} t .
$$

Moreover, by the lower semicontinuity, we have

$$
\begin{aligned}
\int_{0}^{\infty} \int_{\Omega}\left|H_{t}(x)\right|^{2} \mathrm{~d} \mu(x, t) & \leqslant \liminf _{n \rightarrow \infty} \int_{0}^{\infty} \int_{\Omega}\left|\frac{D u_{n} \partial_{t} u_{n}}{e\left(u_{n}\right)}\right|^{2} e\left(u_{n}\right) \mathrm{d} x \mathrm{~d} t \\
& \leqslant\left. 2 \liminf _{n \rightarrow \infty} \int_{0}^{\infty}\left|\int_{\Omega}\right| \partial_{t} u_{n}\right|^{2}(x, t) \mathrm{d} x \mathrm{~d} t<\infty .
\end{aligned}
$$

Here we have used the Schwartz inequality in the last step.

LEMMA 4.5. - For $L^{1}$ a.e. $t \in R_{+}, V_{t}=V_{x, t} \mu_{t} \in V_{m-2}^{*}(\Omega)$ has its first variation $\delta V_{t} \ll \mu_{t}$, and $\delta V_{t}=H_{t} \mu_{t}$ with $H_{t} \in L_{\mu_{t}}^{2}\left(\Omega, R^{m}\right)$.

Proof. - For $Y \in C_{0}^{1}\left(\Omega, R^{m}\right), \gamma \in C_{0}\left(R_{+}, R\right)$. Denote $V_{t}^{n}=V_{A\left(u_{n}\right)(x, t)} \mu_{t}^{n} \in V_{m-2}^{*}(\Omega)$. Then

$$
\begin{aligned}
\int_{R_{+}} \gamma(t) \delta V_{t}^{n}(Y) \mathrm{d} t= & -\int_{R_{+}} \gamma(t) \int_{\Omega} D Y(x): A \mathrm{~d} V_{t}^{n}(x, A) \\
= & -\frac{1}{2} \int_{R_{+}} \gamma(t) \int_{\Omega} D Y(x):\left(\left|D u_{n}\right|^{2} I_{m}-2 D u_{n} \otimes D u_{n}\right)(x, t) \mathrm{d} x \\
& -\int_{\Omega \times R_{+}} \gamma(t) D Y(x): A\left(u_{n}\right)(x, t) \frac{1}{\varepsilon_{n}^{2}} F\left(u_{n}\right)(x, t) \mathrm{d} x \mathrm{~d} t=I+I I .
\end{aligned}
$$

For $I$, multiplying (1.3) by $Y(x) D u_{n}$ and integrating it by parts, we have 


$$
\begin{aligned}
\int_{R_{+}} \gamma(t) \mathrm{d} t \int_{\Omega} Y(x) D u_{n} \partial_{t} u_{n} \\
=\int_{R_{+}} \gamma(t) \mathrm{d} t \int_{\Omega}\left(\Delta u_{n}+\frac{1}{\varepsilon_{n}^{2}} f\left(u_{n}\right)\right) Y(x) D u_{n} \\
=\int_{R_{+}} \gamma(t) \mathrm{d} t \int_{\Omega} \Delta u_{n} Y(x) D u_{n}+\int_{R_{+}} \gamma(t) \mathrm{d} t \int_{\Omega} D\left(\frac{F\left(u_{n}\right)}{\varepsilon_{n}^{2}}\right) Y(x) \\
=\int_{R_{+}} \gamma(t) \mathrm{d} t \int_{\Omega}\left(u_{n, j} u_{n, l} Y^{l}\right)_{j}-Y D\left(\frac{\left|D u_{n}\right|^{2}}{2}\right)-u_{n, j} u_{n, l} Y_{j}^{l} \\
\quad+\int_{R_{+}} \gamma(t) \mathrm{d} t \int_{\Omega} \frac{F\left(u_{n}\right)}{\varepsilon_{n}^{2}} \operatorname{div}(Y) \\
=\int_{R_{+}} \gamma(t) \mathrm{d} t \int_{\Omega}\left(\frac{1}{2}\left|D u_{n}\right|^{2} \operatorname{div}(Y)-u_{n, j} u_{n, l} Y_{j}^{l}\right)+\int_{R_{+}} \gamma(t) \mathrm{d} t \int_{\Omega} \frac{F\left(u_{n}\right)}{\varepsilon_{n}^{2}} \operatorname{div}(Y) .
\end{aligned}
$$

Therefore,

$$
\begin{aligned}
\int_{R_{+}} \gamma(t) \int_{\Omega} \delta V_{t}^{n}(Y) \mathrm{d} t= & -\int_{R_{+}} \gamma(t) \int_{\Omega} Y D u_{n} \partial_{t} u_{n} \\
& -\int_{R_{+}} \gamma(t) \int_{\left\{x \in \Omega:\left|D u_{n}\right|(x, t) \neq 0\right\}} 2 \frac{u_{n, i} u_{n, j} Y_{i}^{j}}{\left|D u_{n}\right|^{2}} \frac{F\left(u_{n}\right)}{\varepsilon_{n}^{2}} .
\end{aligned}
$$

By Lemma 2.4, we know that

$$
\lim _{n \rightarrow \infty} \int_{\Omega \times R_{+}}|\gamma(t)||D Y(x)| \frac{F\left(u_{n}\right)}{\varepsilon_{n}^{2}}(x, t) \mathrm{d} x \mathrm{~d} t=0 .
$$

Therefore, by taking $n$ into infinity, we have

$$
\begin{aligned}
\int_{R_{+}} \gamma(t) \delta V_{t}(Y) \mathrm{d} t & =\lim _{n \rightarrow \infty} \int_{R_{+}} \gamma(t) \mathrm{d} t \int_{\Omega} Y(x)\left(-D u_{n} \partial_{t} u_{n}\right) \mathrm{d} x \mathrm{~d} t \\
& =\int_{R_{+}} \gamma(t) \mathrm{d} t \int_{\Omega}\left\langle H_{t}(x), Y(x)\right\rangle \mathrm{d} \mu_{t} \mathrm{~d} t
\end{aligned}
$$

so that for $L^{1}$ a.e. $t \in R_{+}, \delta V_{t}=H_{t} \mu_{t}$.

For $V \in V_{m-2}^{*}(\Omega)$ and $x \in \Omega$, we define

$$
\Theta^{m-2}(\|V\|, x)=\lim _{r \rightarrow 0} \frac{\|V\|\left(B_{r}(x)\right)}{\alpha(m-2) r^{m-2}} .
$$

Provided that the limit exists and $\alpha(m-2)=\left|B_{1}^{m-2}\right|$. 
Now we state the monotonicity formula for generalized $l$-varifolds $V \in V_{l}^{*}(\Omega)$, whose first variation is a Radon measure. Note that the same formula was shown by Allard (Theorem 5.1 of [6]) for classical $l$-varifolds $V \in V_{l}(\Omega)$.

Lemma 4.6. - Suppose that $V \in V_{l}^{*}(\Omega)$ and $\|\delta V\|$ is a Radon measure on $\Omega$. Then, for any $a \in \operatorname{spt}(\|V\|)$ and $0<r<\operatorname{dist}(a, \partial \Omega)$,

$$
\begin{aligned}
\frac{\mathrm{d}}{\mathrm{d} r}\left(r^{-l}\|V\|\left(B_{r}(a)\right)\right)= & r^{-l-2} \int_{\partial B_{r}(a)}\left|S^{\perp}(x)\right|^{2} \mathrm{~d} V(x, S) \\
& -r^{-l-1} \lim _{\varepsilon \downarrow 0} \delta V\left(\theta_{\varepsilon}(|x|) x\right),
\end{aligned}
$$

where $\theta_{\varepsilon}(|x|) \in C_{0}^{1}\left(B_{r}(a)\right)$ converges to the characteristic function of $B_{r}(a)$ as $\varepsilon \downarrow 0$ and $\left|S^{\perp}(x)\right|^{2}=|x|^{2}-|S(x)|^{2}$.

Proof. - The proof is exactly as same as that by Allard [5] for classical $l$-varifolds. For $\theta_{\varepsilon}(|x|)$ given by the lemma, one has

$$
-\delta V\left(\theta_{\varepsilon}(|x|) x\right)=\int_{B_{r}(a) \times A_{l, m}} \theta^{\prime}(|x|)\left(1-\frac{\left|S^{\perp}(x)\right|^{2}}{|x|}\right) \mathrm{d} V(x, S)+l\|V\|\left(\theta_{\varepsilon}(|x|)\right) .
$$

This can easily seen to imply (4.16).

As a consequence, we obtain the existence of $\Theta^{m-2}\left(\left\|V_{t}\right\|, \cdot\right)$ for $L^{1}$ a.e. $t \in R_{+}$as follows.

COROLlaRY 4.7. - Suppose that $\left\{V_{t}\right\}_{t>0}$ is the family of generalized $(m-2)$ varifolds obtained via (4.10)-(4.14). Then, for $L^{1}$ a.e. $t \in R_{+}$, there exists a set $E_{t} \subset \Omega$, with $H^{m-2}\left(E_{t}\right)=0$, such that $\Theta^{m-2}\left(\left\|V_{t}\right\|, x\right)$ exists for any $x \in \Omega \backslash E_{t}$. Moreover, $\Theta^{m-2}\left(\left\|V_{t}\right\|, \cdot\right)$ is upper semicontinuous for $x \in \Omega \backslash E_{t}$.

Proof. - It follows from Lemma 4.5 that for $L^{1}$ a.e. $t \in R_{+}, H_{t} \in L_{\left\|V_{t}\right\|}^{2}\left(\Omega, R^{m}\right)$, $\delta V_{t}=H_{t}\left\|V_{t}\right\|$, and $\lim _{n \rightarrow \infty} \int_{\Omega}\left|\partial_{t} u_{n}\right|^{2}(x, t)<\infty$. In particular,

$$
\begin{aligned}
\left\|\delta V_{t}\right\|\left(B_{r}(a)\right) & \leqslant \lim _{n \rightarrow \infty} \int_{B_{r}(a)}\left|\partial_{t} u_{n} D u_{n}\right| \\
& \leqslant 2\left(\left\|V_{t}\right\|\left(B_{r}(a)\right)\right)^{\frac{1}{2}} \lim _{n \rightarrow \infty}\left(\int_{B_{r}(a)}\left|\partial_{t} u_{n}\right|^{2}\right)^{\frac{1}{2}} .
\end{aligned}
$$

Hence Lemma 4.6 implies,

$$
\begin{aligned}
\frac{\mathrm{d}}{\mathrm{d} r}\left(r^{2-m}\left\|V_{t}\right\|\left(B_{r}(a)\right)\right) \geqslant & r^{-m-2} \int_{\partial B_{r}(a)}\left|S^{\perp}(x)\right|^{2} \mathrm{~d} V_{t}(x, S) \\
& -2\left(r^{2-m}\left\|V_{t}\right\|\left(B_{r}(a)\right)\right)^{\frac{1}{2}}\left(\lim _{n \rightarrow \infty} r^{2-m} \int_{B_{r}(a)}\left|\partial_{t} u_{n}\right|^{2}\right)^{\frac{1}{2}}
\end{aligned}
$$


F. LIN, C. WANG / Ann. I. H. Poincaré - AN 19 (2002) 209-259

$$
\begin{aligned}
\geqslant & r^{-m-2} \int_{\partial B_{r}(a)}\left|S^{\perp}(x)\right|^{2} \mathrm{~d} V_{t}(x, S)-r^{2-m}\left\|V_{t}\right\|\left(B_{r}(a)\right) \\
& -r^{2-m} \lim _{n \rightarrow \infty} \int_{B_{r}(a)}\left|\partial_{t} u_{n}\right|^{2} .
\end{aligned}
$$

This implies that

$$
\begin{aligned}
\frac{\mathrm{d}}{\mathrm{d} r}\left(\mathrm{e}^{r} r^{2-m}\left\|V_{t}\right\|\left(B_{r}(a)\right)\right) \geqslant & r^{-m-2} \int_{\partial B_{r}(a)}\left|S^{\perp}(x)\right|^{2} \mathrm{~d} V_{t}(x, S) \\
& -r^{2-m} \lim _{n \rightarrow \infty} \int_{B_{r}(a)}\left|\partial_{t} u_{n}\right|^{2} .
\end{aligned}
$$

If we let

$$
E_{t}=\left\{a \in \Omega: \lim _{r \rightarrow 0} r^{2.5-m} \lim _{n \rightarrow \infty} \int_{B_{r}(a)}\left|\partial_{t} u_{n}\right|^{2} \geqslant 1\right\} .
$$

Then, for any $a \in \Omega \backslash E_{t}$, there exists $r_{0}=r_{0}(a)>0$ such that for any $0<r \leqslant r_{0}$

$$
r^{2-m} \lim _{n \rightarrow \infty} \int_{B_{r}(a)}\left|\partial_{t} u_{n}\right|^{2} \leqslant 2 r^{-\frac{1}{2}}
$$

Therefore, if we integrate (4.18) between $0<r_{1} \leqslant r_{2} \leqslant r_{0}$, then we get

$$
\begin{aligned}
& \left(\mathrm{e}^{r_{2}} r_{2}^{2-m}\left\|V_{t}\right\|\left(B_{r_{2}}(a)\right)+\sqrt{r_{2}}\right)-\left(\mathrm{e}^{r_{1}} r_{1}^{2-m}\left\|V_{t}\right\|\left(B_{r_{1}}(a)\right)+\sqrt{r_{1}}\right) \\
& \quad \geqslant \int_{r_{1}}^{r_{2}} r^{-m-2} \int_{\partial B_{r}(a)}\left|S^{\perp}(x)\right|^{2} \mathrm{~d} V_{t}(x, S) .
\end{aligned}
$$

This implies that $\Theta^{m-2}\left(\left\|V_{t}\right\|, a\right)$ exists for all $a \in \Omega \backslash E_{t}$. Moreover it is upper semicontinuous for $a \in \Omega \backslash E_{t}$. Now we want to estimate the size of $E_{t}$ as follows. In fact, a simple Vitali's covering argument implies that $H^{m-2.5}\left(E_{t}\right)<\infty$. In particular, $H^{m-2}\left(E_{t}\right)=0$. This completes the proof.

Note that, by (2.7) and (2.10), $\Sigma_{t}$ has locally finite $(m-2)$-dimensional Hausdorff measure for any $t>0$. Now we have

LEMMA 4.8. - For $L^{1}$ a.e. $t \in R_{+}$, there exists a subset $F_{t} \subset \Sigma_{t}$, with $H^{m-2}\left(F_{t}\right)=0$, such that $\Theta^{m-2}\left(\left\|V_{t}\right\|, x\right) \geqslant \frac{\varepsilon_{0}^{2}}{2}$ for all $x \in \Sigma_{t} \backslash F_{t}$. Here $\varepsilon_{0}$ is given by Lemma 2.3.

Proof. - Define

$$
G=\left\{z \in \Sigma: \lim _{r \downarrow 0} \lim _{n \rightarrow \infty} r^{2-m} \int_{P_{r}(z)}\left|\partial_{t} u_{n}\right|^{2} \geqslant \varepsilon_{0}^{5}\right\} .
$$

Here $\Sigma$ is the concentration set defined in Section 2 and $\varepsilon_{0}$ is given by Lemma 2.3. Then, by the Vitali's covering lemma, we have $\mathcal{P}^{m-2}(G)<\infty$. In particular, $\mathcal{P}^{m}(G)=0$. 
Therefore, for $L^{1}$ a.e. $t \in R_{+}, H^{m-2}\left(G_{t}\right)=0$, here $G_{t}=G \cap\{t\} \subset \Sigma_{t}$. Let $F_{t}=$ $G_{t} \cup\left(E_{t} \cap \Sigma_{t}\right)$, here $E_{t}$ is given by Lemma 4.7. Then it is easy to see $H^{m-2}\left(F_{t}\right)=0$. Now we want to show that for any $a \in \Sigma_{t} \backslash F_{t}, \Theta^{m-2}\left(\left\|V_{t}\right\|, a\right)$ is positive. In fact, there exists $r_{a}>0$ such that

$$
\lim _{n \rightarrow \infty} r^{2-m} \int_{P_{r}(a)}\left|\partial_{t} u_{n}\right|^{2}<\varepsilon_{0}^{5}, \quad \forall 0<r \leqslant r_{a} .
$$

Since $a \in \Sigma_{t}$, it follows from [9] or [31] that for any $r \in\left(0, \frac{r_{a}}{2}\right]$

$$
\lim _{n \rightarrow \infty} r^{2-m} \int_{B_{2 r}(a)} e\left(u_{n}\right)\left(x, t-r^{2}\right) \geqslant \frac{\varepsilon_{0}^{2}}{2} .
$$

On the other hand, Lemma 2.1 implies

$$
\begin{aligned}
\int_{B_{r}(a)} e\left(u_{n}\right)(x, t) \geqslant & \int_{B_{2 r}(a)} e\left(u_{n}\right)\left(x, t-r^{2}\right)-\int_{P_{2 r}(a)}\left|\partial_{t} u_{n}\right|^{2} \\
& -\left(r^{-2} \int_{P_{2 r}(a)}\left|D u_{n}\right|^{2}\right)^{\frac{1}{2}}\left(\int_{P_{2 r}(a)}\left|\partial_{t} u_{n}\right|^{2}\right)^{\frac{1}{2}} \\
& \geqslant \int_{B_{2 r}(a)} e\left(u_{n}\right)\left(x, t-r^{2}\right)-C \varepsilon_{0}^{2.5} r^{m-2} \geqslant \frac{\varepsilon_{0}^{2}}{4} r^{m-2} .
\end{aligned}
$$

Here we have used the fact that $r^{-m} \int_{P_{r}(a)}\left|D u_{n}\right|^{2} \leqslant C$. This implies that, for all $0<r \leqslant$ $r_{a}, \lim _{n \rightarrow \infty} r^{2-m} \int_{B_{r}(a)} e\left(u_{n}\right)(x, t) \geqslant \frac{\varepsilon_{0}^{2}}{4}$. Thus $\Theta^{m-2}\left(\left\|V_{t}\right\|, a\right) \geqslant \frac{\varepsilon_{0}^{2}}{4}$.

THEOREM 4.9. - Under the same notations as above. For $L^{1}$ a.e. $t \in R_{+}, V_{t} \mathrm{~L}\left(\Sigma_{t} \times\right.$ $\left.A_{m-2, m}\right)$ is a $(m-2)$-rectifiable varifold. In particular, $\Sigma_{t}$ is a $(m-2)$-rectifiable set in $\Omega$.

Proof. - One can follow the proof of Theorem 5.5 of Allard [6]. Here we sketch a slightly different proof. First, it follows from Lemma 4.8 that for $L^{1}$ a.e. $t \in R_{+}$, there exists $G_{t} \subset \Sigma_{t}$, with $H^{m-2}\left(G_{t}\right)=0$, such that $\Theta^{m-2}\left(\left\|V_{t}\right\|, x\right)$ is positive and finite for any $x \in \Sigma_{t} \backslash G_{t}$. We can also assume that $H^{m-2}\left(\Sigma_{t} \backslash G_{t}\right)>0$ (otherwise, we have nothing to prove). Moreover, since $\Theta^{m-2}\left(\left\|V_{t}\right\|, \cdot\right)$ is upper semicontinuous on $\Sigma_{t} \backslash G_{t}, \Theta^{m-2}\left(\left\|V_{t}\right\|, x\right)$ is $H^{m-2}$-approximately continuous for $H^{m-2}$ a.e. in $\Sigma_{t} \backslash G_{t}$. If we represent $V_{t}=V_{x, t}\left\|V_{t}\right\|$, with $V_{x, t}$ a probability measure on $A_{m-2, m}$, then $V_{x, t}$ is a $H^{m-2}$-measurable function with valued in the space of probability measures on $A_{m-2, m}$. It is well-known that $V_{x, t}$ is $H^{m-2}$-approximately continuous for $H^{m-2}$ a.e. $x \in \Sigma_{t}$. Therefore, for $H^{m-2}$ a.e. $x_{0} \in \Sigma_{t}$, the following four properties hold:

$$
\Theta^{*, m-2}\left(\Sigma_{t}, x_{0}\right)=\limsup _{r \downarrow 0} r^{2-m} H^{m-2}\left(\Sigma_{t} \cap B_{r}\left(x_{0}\right)\right) \geqslant 2^{-m-2},
$$




$$
\begin{gathered}
V_{x, t} \text { is } H^{m-2} \text { approximately continuous at } x_{0}, \\
\lim _{r \downarrow 0} \frac{\int_{B_{r}\left(x_{0}\right)}\left|H_{t}\right| \mathrm{d}\left\|V_{t}\right\|}{r^{m-2}}=\left|H_{t}\left(x_{0}\right)\right| \Theta^{m-2}\left(\left\|V_{t}\right\|, x_{0}\right)<\infty .
\end{gathered}
$$

Based on these and the Geometric Lemma 2.4 of [23], we can assure that for any $r_{i} \downarrow 0$, there exists a subsequence $r_{i^{\prime}} \downarrow 0$ such that

$$
\mathcal{D}_{x_{0}, r_{i^{\prime}}}\left(V_{x, t}\right) \rightarrow V_{x_{0}, t} H^{m-2} \mathrm{~L} T
$$

for some $(m-2)$-plane $T \subset R^{m}$, Now we want to show that $T$ is independent of the choice of $\left\{i^{\prime}\right\}$. In fact, by (4.22), we have

$$
\lim _{i^{\prime} \rightarrow \infty}\left\|\delta\left(\mathcal{D}_{x_{0}, r_{i^{\prime}}}\left(V_{x, t}\right)\right)\right\|=\lim _{r^{\prime} \rightarrow \infty} r_{i^{\prime}}^{3-m}\left(\mathcal{D}_{x_{0}, r_{i^{\prime}}}\right)_{\#}\left\|\delta V_{x, t}\right\|=0 .
$$

Therefore

$$
\delta\left(V_{x_{0}, t} H^{m-2} \mathrm{~L} T\right)=0
$$

so that the constancy theorem for varifolds (see, Simon [33]) implies that $V_{x_{0}, t}=\delta_{T}$, i.e. the Dirac mass at $T$. In particular, $T$ is unique. This proves that $V_{t} \mathrm{~L}\left(\Sigma_{t} \times A_{m-2, m}\right)$ is a $(m-2)$-rectifiable varifold. In particular, $\Sigma_{t}=\operatorname{spt}\left(\left\|V_{t}\right\|\right)$ is a $H^{m-2}$-rectifiable set.

Finally, we derive some consequences of the Theorem 4.9. Let us first consider the critical points of the Ginzburg-Landau functional.

COROLLARY 4.10 (Continuation of Example 4.4). - Under the same assumptions as in Example 4.4. There exist a closed $(m-2)$-rectifiable set $\Sigma \subset \Omega$ and a $H^{m-2}$ measurable function $\varepsilon_{0}^{2} \leqslant \theta<\infty$ on $\Omega$ such that

(1) $v(x)=\theta(x) H^{m-2} L \Sigma$ for $H^{m-2}$ a.e. $x \in \Sigma$, and

$$
V_{u_{n}} \rightarrow V \equiv V_{u}+V(\Sigma, \theta)
$$

as convergences of generalized $(m-2)$-varifolds on $\Omega$, here

$$
V(\Sigma, \theta)=\delta_{T_{x} \Sigma} \theta H^{m-2} \mathrm{~L} \Sigma .
$$

Moreover, $V$ is stationary, i.e., for any $Y \in C_{0}^{1}\left(\Omega, R^{m}\right)$,

$$
\int_{\Omega} \frac{1}{2}|D u|^{2} \operatorname{div}(Y)-\sum_{1 \leqslant i j \leqslant m} u_{i} u_{j} Y_{i}^{j}+\int_{\Sigma} \operatorname{div}_{\Sigma}(Y) \theta \mathrm{d} H^{m-2}=0 .
$$

(2) If, in addition, $N=S^{k-1}$. Then

$$
\theta(x)=\sum_{i=1}^{l_{x}} E\left(\phi_{j}, S^{2}\right), \quad \text { for } H^{m-2} \text { a.e. } x \in \Sigma .
$$

Here $1 \leqslant l_{x}<\infty$ and $\phi_{j}: S^{2} \rightarrow S^{k-1}$ are nontrivial harmonic maps for $1 \leqslant j \leqslant l_{x}$. 
(3) If, in addition, $N=S^{2}$. Then $\theta(x)=4 \pi n_{x}$ for some positive integer $n_{x}$, for $H^{m-2}$ a.e. $x \in \Sigma$. In particular, $\frac{1}{4 \pi} V(\Sigma, \theta)$ is an integral $(m-2)$-varifold.

Proof. - It follows from the static versions of Lemmas 2.1-2.3 for $u_{n}$ that the concentration set is given by

$$
\Sigma=\left\{x \in \Omega: \varepsilon_{0}^{2} \leqslant \Theta^{m-2}(\|V\|, x)<\infty\right\}=\left\{x \in \Omega: \Theta^{m-2}(\|V\|, x)>0\right\} .
$$

Moreover, as in Example 4.4, $\delta V=0$. Therefore, Theorem 4.9 implies

$$
V \mathrm{~L}\left\{(x, A): \Theta^{m-2}(\|V\|, x)>0, A \in A_{m-2, m}\right\}=\delta_{T_{x} \Sigma} \Theta^{m-2}(\|V\|, x) H^{m-2} \mathrm{~L} \Sigma
$$

is a $(m-2)$-rectifiable varifold. In particular, $\Sigma$ is a $(m-2)$-rectifiable set. Moreover, since $u_{n} \rightarrow u$ in $C_{\text {loc }}^{1}\left(\Omega \backslash \Sigma, R^{k}\right)$, we have

$$
V \mathrm{~L}(\Omega \backslash \Sigma) \times A_{m-2, m}=\frac{1}{2} \delta_{A(u)}|D u|^{2}(x) \mathrm{d} x .
$$

Therefore, we obtain (4.24) and (4.25). This proves (1).

The conclusion of (2) comes from the Theorem B of [32] (one can also see Section 6 below). Part (3) follows from (2) and the fact that any nontrivial harmonic map from $S^{2}$ to $S^{2}$ has energy equal to $4 \pi n$ for some positive integer $n$.

Recall that a stationary harmonic map $u \in H^{1}(\Omega, N)$ is a weakly harmonic map, which satisfies

$$
\int_{\Omega}|D u|^{2} \operatorname{div}(X)-2 \sum_{1 \leqslant i j \leqslant m} u_{i} u_{j} X_{j}^{i}=0, \quad \forall X \in C_{0}^{1}\left(\Omega, R^{m}\right) .
$$

By quoting the result by Lin and Riviere [27], we can obtain

COROLlaRY 4.11. - Let $\left\{u_{n}\right\} \subset H^{1}(\Omega, N)$ be stationary harmonic maps. Assume that $u_{n} \rightarrow u$ weakly in $H^{1}(\Omega, N), \frac{1}{2}\left|D u_{n}\right|^{2}(x) \mathrm{d} x \rightarrow \frac{1}{2}|D u|^{2}(x) \mathrm{d} x+v$ for some nonnegative Radon measure $v$ on $\Omega$, and $V_{u_{n}} \rightarrow V$ as convergence of generalized $(m-2)$-varifolds. Then

(1) There exist a $(m-2)$-rectifiable close set $\Sigma \subset \Omega$ and a $H^{m-2}$ measurable function $\varepsilon_{0}^{2} \leqslant \theta<\infty$ on $\Omega$ such that $v(x)=\theta(x) H^{m-2} \mathrm{~L} \Sigma(x)$ for $H^{m-2}$ a.e. $x \in \Sigma$.

(2) $V=V_{u}+V(\Sigma, \theta)$ and is stationary, i.e., for any $Y \in C_{0}^{1}\left(\Omega, R^{m}\right)$,

$$
\int_{\Omega} \frac{1}{2}|D u|^{2} \operatorname{div} Y-\sum_{1 \leqslant i j \leqslant m} u_{i} u_{j} Y_{i}^{j}+\int_{\Sigma} \operatorname{div}_{\Sigma} Y \theta \mathrm{d} H^{m-2}=0 .
$$

(3) If, in addition, $N=S^{k-1}$. Then $\theta(x)=\sum_{i=1}^{l_{x}} E\left(\phi_{j}, S^{2}\right)$ for $H^{m-2}$ a.e. $x \in \Sigma$, here $1 \leqslant l_{x}<\infty$ and $\phi_{j}: S^{2} \rightarrow S^{k-1}$ is a nontrivial harmonic map. Furthermore, if $k=3$, then $\theta(x)=4 \pi n_{x}$ for some positive integer $n_{x}$, for $H^{m-2}$ a.e. $x \in \Sigma$. In particular, $\frac{1}{4 \pi} V(\Sigma, \theta)$ is an integral $(m-2)$-varifold. 


\section{Generalized varifold flows and the Brakke flow}

In this section, we will prove that the limiting pair $\left(u, v_{t} \mathrm{~d} t\right)$ satisfies the generalized varifold flow, which will be defined below. The generalized varifold flow implies that $\left\{v_{t}\right\}_{t \geqslant 0}$ is a Brakke flow of $(m-2)$-rectifiable varifolds, under the extra assumption that $u$ is in the class of "suitably weak solutions" to the heat flow of harmonic maps, which requires that the energy equality (2.1) holds. Similar notion of suitably weak solutions to the Navie-Stokes equations was introduced by Cafferalli, Nirenberg and Kohn [12]. A stronger class of weak solutions behaving like parabolic stationary harmonic maps was introduced by Chen, Li and Lin [10] and Feldman [17].

We will use the same notations in Section 4 throughout this section. We first apply Theorem 4.9 to express the varifold $V_{t}$ for $L^{1}$ a.e. $t>0$.

LEMMA 5.1. - For $L^{1}$ a.e. $t>0$, we have

$$
V_{t}=\frac{1}{2} \delta_{A(u(\cdot, t))}|D u|^{2}(x, t) \mathrm{d} x+V\left(\Sigma_{t}, \Theta^{m-2}\left(\left\|V_{t}\right\|, \cdot\right)\right) .
$$

Proof. - It follows from Lemmas 4.5-4.8 that for $L^{1}$ a.e. $t>0, \delta V_{t}=H_{t}\left\|V_{t}\right\|$, $H_{t} \in L_{\left\|V_{t}\right\|}^{2}\left(\Omega, R^{m}\right), \varepsilon_{0}^{2} \leqslant \Theta^{m-2}\left(\left\|V_{t}\right\|, x\right)<\infty$ for $H^{m-2}$ a.e. $x \in \Sigma_{t}$, here $\Sigma_{t}=\Sigma \cap\{t\}$ and $\Sigma$ is defined by Section 2. Therefore, Theorem 4.9 implies that $V_{t} \mathrm{~L}\left(\Sigma_{t} \times A_{m-2, m}\right)$ is a $(m-2)$-varifold and

$$
V_{t} \mathrm{~L} \Sigma_{t}=\delta_{T_{x} \Sigma_{t}} \Theta^{m-2}\left(\left\|V_{t}\right\|, x\right) H^{m-2} \mathrm{~L} \Sigma_{t}=V\left(\Sigma_{t}, \Theta^{m-2}\left(\left\|V_{t}\right\|, \cdot\right)\right) .
$$

Since, on $\Omega \backslash \Sigma_{t}$, we have $u_{n} \rightarrow u$ in $C_{\text {loc }}^{1}$ so that $V_{u_{n}(\cdot, t)} \rightarrow V_{u(\cdot, t)}$ on $\Omega \backslash \Sigma_{t}$. Therefore,

$$
V_{t} \mathrm{~L}\left(\Omega \backslash \Sigma_{t}\right)=\frac{1}{2} \delta_{A(u(\cdot, t))}|D u|^{2}(x, t) \mathrm{d} x .
$$

Combining these two facts, we obtain (5.1).

The next Lemma shows that generically $H_{t}(x) \in\left(T_{x} \Sigma_{t}\right)^{\perp}$.

LEMMA 5.2. - For $L^{1}$ a.e. $t>0$, we have

$$
H_{t}(x) \perp T_{x} \Sigma_{t}, \quad \text { for } H^{m-2} \text { a.e. } x \in \Sigma_{t} .
$$

Proof. - This can be proved by the Young measure method. Let $M^{m k}$ denote the set of $m \times k$ matrices and consider Radon measures $W_{n}$ on $\Omega \times R_{+} \times M^{m k}$ by

$$
W_{n}(x, t, A)=\delta_{\frac{D u_{n}}{\left|D u_{n}\right|}(x, t)}(A) e\left(u_{n}\right)(x, t) \mathrm{d} x \mathrm{~d} t .
$$

Define $\phi: M^{m k} \rightarrow A_{m-2, m}$ by $\phi(A)=I_{m}-2 A^{t} A$. Then we see that $\phi_{\#}\left(W_{n}\right)=V_{u_{n}}$, here $V_{u_{n}}$ is the generalized ( $m-2$ )-varifold on $\Omega \times R_{+} \times A_{m-2, m}$ defined in Section 4. Since we can assume that $V_{u_{n}} \rightarrow V=V_{x, t} \mu_{t} \mathrm{~d} t$ and $W_{n} \rightarrow W=W_{x, t} \mu_{t} \mathrm{~d} t$ for some probability measures $V_{x, t}$ on $A_{m-2, m}$, and $W_{x, t}$ on $M^{m k}$, we then have $V_{x, t}=\phi_{\#}\left(W_{x, t}\right)$. Since, for 
$L^{1}$ a.e. $t>0$, Lemma 5.1 holds. Therefore, for $L^{1}$ a.e. $t>0, V_{x, t}=\delta_{T_{x} \Sigma_{t}}$ for $x \in \Sigma_{t}$. In particular, for $H^{m-2}$ a.e. $x \in \Sigma_{t}$,

$$
\int_{M^{m k}}\left(I_{m}-2 A^{t} A\right) \mathrm{d} W_{x, t}(A)=\int_{A_{m-2, m}} A \mathrm{~d} V_{x, t}(A)=T_{x} \Sigma_{t} .
$$

For any unit vector $e \in T_{x} \Sigma_{t}$, we then have

$$
\begin{aligned}
1 & =\left\langle T_{x} \Sigma_{t}(e), e\right\rangle \\
& =\left\langle e, \int_{M^{m k}}\left(I_{m}-2 A^{t} A\right) \mathrm{d} W_{x, t}(A)(e)\right\rangle \\
& =1-2 \int_{M^{m k}}|A(e)|^{2} \mathrm{~d} W_{x, t}(A) .
\end{aligned}
$$

Hence, for $H^{m-2}$ a.e. $x \in \Sigma_{t},|A(e)|=0$ for $W_{x, t}$ a.e. $A \in M^{m k}$. This implies that for $H^{m-2}$ a.e. $x \in \Sigma_{t}$, the support of $W_{x, t}$ is contained in $E(A) \equiv\left\{A=\left(A_{1}, \ldots, A_{k}\right)^{t}\right.$ : $\left.\operatorname{span}\left\{A_{1}, \ldots, A_{k}\right\} \subset\left(T_{x} \Sigma_{t}\right)^{\perp}\right\}$. Note also that if we define $Z_{n}=\delta_{\frac{D u_{n}}{\left|D u_{n}\right|}} \partial_{t} u_{n} D u_{n} \mathrm{~d} x \mathrm{~d} t$, then $Z_{n} \ll W_{n}$. Therefore, if we assume that $Z_{n} \rightarrow Z$ on $\Omega \times R_{+} \times M^{m k}$, then $Z \ll W$ and there exists a vector valued function $Z_{x, t}$ on $M^{m k}$ such that $Z=Z_{x, t} W_{x, t} \mu_{t} \mathrm{~d} t$. Since $\left(\pi_{x, t}\right)_{\#} Z_{n}=\partial_{t} u_{n} D u_{n} \mathrm{~d} x \mathrm{~d} t \rightarrow-H_{t}(x) \mu_{t} \mathrm{~d} t$, we have

$$
-H_{t}(x)=\int_{M^{m k}} Z_{x, t}(A) \mathrm{d} W_{x, t}(A) .
$$

We now claim that for $H^{m-2}$ a.e. $x \in \Sigma_{t}, Z_{x, t}(A) \in \operatorname{spt}\left(W_{x, t}\right)$, which clearly implies $H_{t}(x) \in\left(T_{x} \Sigma_{t}\right)^{\perp}$. In fact, since $\partial_{t} u_{n} D u_{n} \in E\left(\frac{D u_{n}}{\left|D u_{n}\right|}\right)$, we have

$$
\int_{M^{m k}} \operatorname{dist}\left(A, \frac{\mathrm{d} Z_{n}}{\mathrm{~d}\left\|Z_{n}\right\|}\right) \mathrm{d}\left\|Z_{n}\right\|(A)=0
$$

taking $n$ into infinity and by the lower semicontinuity, this gives

$$
\int_{M^{m k}} \operatorname{dist}\left(A, \frac{\mathrm{d} Z}{\mathrm{~d}\|Z\|}\right) \mathrm{d}\|Z\|=0 .
$$

This also implies that for $W_{x, t}$ a.e. $A \in M^{m k}, Z_{x, t}(A) \in \operatorname{spt}\left(W_{x, t}\right)$.

Now we prove an energy inequality for the limiting Radon measures $v, \eta$. Assume that

$$
\begin{gathered}
e\left(u_{n}\right)(x, t) \mathrm{d} x \rightarrow \frac{1}{2}|D u|^{2}(x, t) \mathrm{d} x+v_{t}, \\
\left|\partial_{t} u_{n}\right|^{2}(x, t) \mathrm{d} x \mathrm{~d} t \rightarrow\left|\partial_{t} u\right|^{2}(x, t) \mathrm{d} x \mathrm{~d} t+\eta
\end{gathered}
$$

for some Radon measures $\left\{v_{t}\right\}_{t>0}$ on $\Omega$ and $\eta$ on $\Omega \times R_{+}$. Then 
PROPOSITION 5.3 (Energy inequality). - Under the same notations as above. We have, for any $0<t_{1}<t_{2}<\infty$ and $\phi \in C_{0}^{1}\left(\Omega, R_{+}\right)$,

$$
\begin{aligned}
\int_{\Omega} & \frac{1}{2} \phi(x)|D u|^{2}\left(x, t_{2}\right)+v_{t_{2}}(\phi)-\int_{\Omega} \frac{1}{2} \phi(x)|D u|^{2}\left(x, t_{1}\right)-v_{t_{1}}(\phi) \\
\leqslant & -\int_{\Omega \times\left[t_{1}, t_{2}\right]}\left(\left|\partial_{t} u\right|^{2} \phi+D \phi \partial_{t} u D u\right) \\
& -\int_{\Sigma_{t_{1}}^{t_{2}}}\left(\phi d \eta-\left\langle\left(T_{x}\left(\Sigma_{t}\right)\right)^{\perp} D \phi, H_{t}\right\rangle \mathrm{d}\left\|V_{t}\right\| \mathrm{d} t .\right.
\end{aligned}
$$

Here $v_{t}(\phi)=\int_{\Omega} \phi(x) \mathrm{d} v_{t}(x), \Sigma_{t_{1}}^{t_{2}}=\Sigma \cap R^{m} \times\left[t_{1}, t_{2}\right]$, and $\left(T_{x} \Sigma_{t}\right)^{\perp}$ denotes the normal space of $\Sigma_{t}$ at $x$.

Proof. - By taking $n$ into infinity in the equality (2.1), we have

$$
\begin{gathered}
\int_{\Omega} \frac{1}{2} \phi(x)|D u|^{2}\left(x, t_{2}\right)+v_{t_{2}}(\phi)-\int_{\Omega} \frac{1}{2} \phi(x)|D u|^{2}\left(x, t_{1}\right)-v_{t_{1}}(\phi) \\
=-\int_{\Omega \times\left[t_{1}, t_{2}\right]} \phi\left|\partial_{t} u\right|^{2}-\int_{\Sigma_{t_{1}}} \phi \mathrm{d} \eta+\int_{\Omega \times\left[t_{1}, t_{2}\right]}\left\langle D \phi, H_{t}\right\rangle \mathrm{d}\left\|V_{t}\right\| \mathrm{d} t .
\end{gathered}
$$

Sine $\partial_{t} u_{n} D u_{n} \rightarrow \partial_{t} u D u$ strongly in $L_{\text {loc }}^{2}\left(\Omega \times R_{+} \backslash \Sigma\right), H_{t} \mathrm{~d} \mu_{t}=-\partial_{t} u D u \mathrm{~d} x$ on $\Omega \times R_{+} \backslash \Sigma$. Therefore, by Lemma 5.2, we have

$$
\begin{aligned}
\int_{\Omega \times\left[t_{1}, t_{2}\right]}\left\langle D \phi, H_{t}\right\rangle \mathrm{d}\left\|V_{t}\right\| \mathrm{d} t & =-\int_{\Omega \times\left[t_{1}, t_{2}\right]} D \phi \partial_{t} u D u+\int_{\Sigma_{t_{1}}^{t_{2}}}\left\langle D \phi, H_{t}\right\rangle \mathrm{d} \mu_{t} \\
& =-\int_{\Omega \times\left[t_{1}, t_{2}\right]} D \phi \partial_{t} u D u+\int_{\Sigma_{t_{1}}^{t_{2}}}\left\langle\left(T_{x} \Sigma_{t}\right)^{\perp} D \phi, H_{t}\right\rangle \mathrm{d} \mu_{t} .
\end{aligned}
$$

This gives (5.3).

COROLlARY 5.4. - Under the same notations as above. We have, for any $0<t_{1}<$ $t_{2}<\infty$ and $\phi \in C_{0}^{1}\left(\Omega, R_{+}\right)$,

$$
\begin{gathered}
\int_{\Omega} \frac{1}{2} \phi(x)|D u|^{2}\left(x, t_{2}\right)+v_{t_{2}}(\phi)-\int_{\Omega} \frac{1}{2} \phi(x)|D u|^{2}\left(x, t_{1}\right)-v_{t_{1}}(\phi) \\
\leqslant-\int_{\Omega \times\left[t_{1}, t_{2}\right]}\left(\left|\partial_{t} u\right|^{2} \phi+D \phi \partial_{t} u D u\right) \\
\quad-\int_{\Sigma^{t_{2}}}\left(\frac{1}{2}\left|H_{t}\right|^{2} \phi-\left\langle\left(T_{x}\left(\Sigma_{t}\right)\right)^{\perp} D \phi, H_{t}\right\rangle\right) \mathrm{d}\left\|V_{t}\right\| \mathrm{d} t .
\end{gathered}
$$


Proof. - It suffices to prove

$$
\int_{\Sigma_{t_{1}}^{t_{2}}} \phi \mathrm{d} \eta \geqslant \frac{1}{2} \int_{\Sigma_{t_{1}}^{t_{2}}}\left|H_{t}\right|^{2} \phi \mathrm{d} v_{t} \mathrm{~d} t .
$$

To see it, we note that, for $\mathcal{P}^{m}$ a.e. $z_{0}=\left(x_{0}, t_{0}\right) \in \Sigma$, by the Schwartz inequality, we have

$$
\begin{aligned}
\left|H_{t_{0}}\left(x_{0}\right)\right|^{2} & \leqslant \lim _{r \downarrow 0} \lim _{n \rightarrow \infty}\left(\frac{\left|\int_{P_{r}\left(z_{0}\right)} \partial_{t} u_{n} D u_{n}\right|}{\int_{P_{r}\left(z_{0}\right)} e\left(u_{n}\right)}\right)^{2} \\
& \leqslant 2 \lim _{r \downarrow 0} \lim _{n \downarrow \infty} \frac{\int_{P_{r}\left(z_{0}\right)}\left|\partial_{t} u_{n}\right|^{2}}{\int_{P_{r}\left(z_{0}\right)} e\left(u_{n}\right)} .
\end{aligned}
$$

Hence

$$
\begin{aligned}
\left|H_{t_{0}}\left(x_{0}\right)\right|^{2} \mathrm{~d} \mu\left(z_{0}\right) & \leqslant 2 \lim _{r \downarrow 0} \lim _{n \rightarrow \infty} \int_{P_{r}\left(z_{0}\right)}\left|\partial_{t} u_{n}\right|^{2} \\
& =2 \lim _{r \rightarrow 0}\left(\int_{P_{r}\left(z_{0}\right)}\left|\partial_{t} u\right|^{2}+\eta\left(P_{r}\left(z_{0}\right)\right)\right) \\
& \leqslant 2 \mathrm{~d} \eta\left(z_{0}\right) .
\end{aligned}
$$

This gives (5.6).

Now we give the definition of generalized varifold flow for a pair $\left(v, \eta_{t} \mathrm{~d} t\right)$.

DEFINITION 5.5. - Let $v \in H_{\mathrm{loc}}^{1}\left(\Omega \times R_{+}, N\right) \cap L^{\infty}\left(R_{+}, H^{1}(\Omega, N)\right)$ and $\left\{\eta_{t}\right\}_{t \geqslant 0}$ be nonnegative Radon measures on $\Omega$. We say that the pair $\left(v, \eta_{t} \mathrm{~d} t\right)$ is a generalized varifold flow, if the following holds

(1) $v$ is a weak solution to the heat equation of harmonic maps, i.e. satisfies (1.0) in the sense of distribution.

(2) For $L^{1}$ a.e. $t \in R_{+}, \eta_{t}=\left\|V_{t}\right\|$ for some $(m-2)$-rectifiable varifold $V_{t} \in V_{m-2}(\Omega)$, $\delta V_{t}=H_{t}\left\|V_{t}\right\|$, and $H_{t} \in L_{\left\|V_{t}\right\|}^{2}\left(\Omega, R^{m}\right)$.

(3) For any $0 \leqslant s \leqslant t<\infty$ and $\phi \in C_{0}^{1}\left(\Omega, R_{+}\right)$, we have

$$
\begin{aligned}
\int_{\Omega} & \frac{1}{2}|D v|^{2}(x, t) \phi(x)+\eta_{t}(\phi)-\int_{\Omega} \frac{1}{2}|D v|^{2}(x, s) \phi(x)-\eta_{s}(\phi) \\
\leqslant & -\int_{s}^{t} \int_{\Omega}\left(\left|\partial_{t} v\right|^{2} \phi+D \phi \partial_{t} v D v\right) \\
& -\int_{s}^{t} \int_{A_{t}}\left(\phi(x)\left|H_{t}(x)\right|^{2}-\left\langle\left(T_{x} A_{t}\right)^{\perp} D \phi, H_{t}(x)\right\rangle\right) \mathrm{d} \eta_{t} \mathrm{~d} t .
\end{aligned}
$$

Here $A_{t}=\operatorname{spt}\left(\eta_{t}\right)$.

One of the main theorem of this section is to show that the limiting pair $\left(u, v_{t} \mathrm{~d} t\right)$ obtained from the limiting process of sequences of solutions to the heat flow of the Ginzburg-Landau functional, i.e. solutions to (1.3)-(1.4), is a generalized varifold flow. 
THEOREM 5.6. - Under the same notations as above. The limiting pair $\left(u, v_{t} \mathrm{~d} t\right)$ is a generalized varifold flow.

Proof. - By comparing (5.5) and (5.7), we know that in order to show the pair $\left(u, v_{t} \mathrm{~d} t\right)$ is a generalized varifold flow, it suffices to improve the $\frac{1}{2}$ factor in front of the term $\int_{s}^{t} \int_{\Sigma_{t}} \phi(x)\left|H_{t}(x)\right|^{2}$ of (5.5) to 1 . In other word, we need to prove

LEMMA 5.7. - Under the same notations as above.

$$
\int_{\Sigma_{t}^{s}} \phi(x)\left|H_{t}(x)\right|^{2} \mathrm{~d} v_{t}(x) \mathrm{d} t \leqslant \int_{\Sigma_{t}^{s}} \phi(x) \mathrm{d} \eta(x, t)
$$

for any $0<t \leqslant s<\infty$ and $\phi \in C_{0}^{1}\left(\Omega, R_{+}\right)$.

Before we prove Lemma 5.7, we would like to remark that (5.8) also follows from the energy quantization Theorem 6.1 of Section 6 below, which is only proved at present under the assumption that $N=S^{k-1}$ however. Here we present a different proof of it, which is valid for all manifold $N$.

LemMA 5.8. - For $\mathcal{P}^{m}$ a.e. $z=(x, t) \in \Sigma$, we have

$$
\begin{gathered}
\lim _{r \downarrow 0} \lim _{n \rightarrow \infty} r^{-m} \int_{P_{r}(z)}\left(\left|D_{x} u_{n}\right|^{2}-\left|D_{y} u_{n}\right|^{2}\right) \mathrm{d} x \mathrm{~d} t=0, \\
\lim _{r \downarrow 0} \lim _{n \rightarrow \infty} r^{-m} \int_{P_{r}(z)} D_{x} u_{n} D_{y} u_{n} \mathrm{~d} x \mathrm{~d} t=0 .
\end{gathered}
$$

Here $(x, y)$ is the coordinate function of $\left(T_{x} \Sigma_{t}\right)^{\perp}$.

Proof. - First note from the proof of Theorem 6.1 of Section 6 below that for $\mathcal{P}^{m}$ a.e. $z_{0}=\left(x_{0}, t_{0}\right) \in \Sigma$, the following properties hold:

$$
\begin{gathered}
\lim _{r \downarrow 0} \lim _{n \rightarrow \infty} r^{-m} \int_{P_{r}\left(z_{0}\right)} e\left(u_{n}\right)=\Theta^{m-2}\left(\left\|V_{t_{0}}\right\|, x_{0}\right), \\
\lim _{r \downarrow 0} r^{2-m} \int_{P_{r}\left(z_{0}\right)}\left|\partial_{t} u_{n}\right|^{2}=0, \\
\lim _{r \downarrow 0}\left(r^{2-m} \int_{P_{r}\left(z_{0}\right)}\left|\partial_{t} u\right|^{2}+r^{-m} \int_{P_{r}\left(z_{0}\right)}|D u|^{2}\right)=0, \\
\lim _{r \downarrow 0} \lim _{n \rightarrow \infty} r^{-m} \int_{P_{r}\left(z_{0}\right)}\left|D_{z} u_{n}\right|^{2}=0,
\end{gathered}
$$

where $z$ is any vector in the $(m-2)$-plane $T_{x_{0}} \Sigma_{t_{0}} \subset R^{m}$. 
For any such $z_{0}=\left(x_{0}, t_{0}\right)$, we identify $T_{x_{0}} \Sigma_{t_{0}}=\{(0,0)\} \times R^{m-2}$ and write the coordinate of $R^{m}$ as $R^{m}=\left\{(x, y, z):(x, y) \in R^{2}, z \in R^{m-2}\right\}$.

For $r_{n} \downarrow 0$, let $v_{n}(x, t)=u_{n}\left(x_{0}+r_{n} x, t_{0}+r_{n}^{2} t\right): P_{2}(0) \rightarrow R^{k}$. Then we know that $v_{n}$ solves (1.3), with $\varepsilon_{n}$ replaced by $\bar{\varepsilon}_{n}=\frac{\varepsilon_{n}}{r_{n}} \rightarrow 0$, and

$$
\begin{gathered}
v_{n}(x, y, z, t) \rightarrow \text { constant in } C_{\text {loc }}^{1}\left(R^{m+1} \backslash R^{m-2} \times R, R^{k}\right), \\
e\left(v_{n}\right)(x, y, z, t) \mathrm{d} x \mathrm{~d} y \mathrm{~d} z \mathrm{~d} t \rightarrow \Theta^{m-2}\left(\left\|V_{t_{0}}\right\|, x_{0}\right)\left(H^{m-2} \mathrm{~L} R^{m-2}\right) \times\left(L^{1} \mathrm{~L} R\right), \\
\left(\left|D_{x} v_{n}\right|^{2}-\left|D_{y} v_{n}\right|^{2}\right)(x, y, z, t) \mathrm{d} x \mathrm{~d} y \mathrm{~d} z \mathrm{~d} t \rightarrow \alpha(z, t) H^{m-2} \mathrm{~L} R^{m-2} \times L^{1} \mathrm{~L} R, \\
D_{x} v_{n} D_{y} v_{n}(x, y, z, t) \mathrm{d} x \mathrm{~d} y \mathrm{~d} z \mathrm{~d} t \rightarrow \beta(z, t) H^{m-2} \mathrm{~L} R^{m-2} \times L^{1} \mathrm{~L} R
\end{gathered}
$$

as convergences of Radon measures on $P_{2}(0)$, for some measurable functions $\alpha, \beta$ on $R^{m-2} \times R$. Observe that (5.9) and (5.10) are equivalent to

$$
\int_{B_{1}^{m-2} \times(-1,1)} \alpha(z, t) \mathrm{d} z \mathrm{~d} t=\int_{B_{1}^{m-2} \times(-1,1)} \beta(z, t) \mathrm{d} z \mathrm{~d} t=0 .
$$

In order to prove (5.18), we need the Pohozaev identity for $v_{n}$ as follows. For $X \in$ $C_{0}^{1}\left(B_{2}^{m}, R^{m}\right)$, multiplying the equations of $v_{n}$ by $X(x, y, z) D v_{n}(x, y, z)$, we get

$$
\int_{P_{2}(0)} \partial_{t} v_{n} D v_{n} X=\int_{P_{2}(0)} e\left(v_{n}\right) \operatorname{div}(X)-\sum_{i, j} v_{n, i} v_{n, j} X_{i}^{j} .
$$

Note that (5.12) implies

$$
\lim _{n \rightarrow \infty} \int_{P_{2}} \partial_{t} v_{n} D v_{n} X=0
$$

and Lemma 2.4 implies

$$
\int_{P_{2}(0)} e\left(v_{n}\right) \operatorname{div}(X)=\int_{P_{2}(0)} \frac{1}{2}\left|D v_{n}\right|^{2} \operatorname{div}(X)+\mathrm{O}\left(n^{-1}\right) .
$$

Hence, for $X=\left(X^{1}, X^{2}, \ldots, X^{m}\right) \in C_{0}^{1}\left(B_{2}^{m}, R^{m}\right)$, we have

$$
\begin{aligned}
\int_{P_{2}(0)} \frac{1}{2}\left|D v_{n}\right|^{2} \operatorname{div}(X)= & \int_{P_{2}(0)} \frac{1}{2}\left|D v_{n}\right|^{2}\left(X_{x}^{1}+X_{y}^{2}\right)+\sum_{j=3}^{m} \int_{P_{2}(0)} \frac{1}{2}\left|D v_{n}\right|^{2} X_{j}^{j} \\
& -\sum_{3 \leqslant i j \leqslant m_{P_{2}(0)}} \int_{3, i} v_{n, i} v_{n, j} X_{i}^{j}-\sum_{3 \leqslant i \leqslant m_{P_{2}(0)}} v_{n, i}\left(v_{n, x} X_{i}^{1}+v_{n, y} X_{i}^{2}\right) \\
& -\sum_{3 \leqslant j \leqslant m_{P_{2}(0)}} \int_{n, j} v_{n, j}\left(v_{n, x} X_{x}^{j}+v_{n, y} X_{y}^{j}\right)
\end{aligned}
$$


F. LIN, C. WANG / Ann. I. H. Poincaré - AN 19 (2002) 209-259

$$
\begin{aligned}
& -\int_{P_{2}(0)}\left(\left|v_{n, x}\right|^{2} X_{x}^{1}+\left|v_{n, y}\right|^{2} X_{y}^{2}\right)+v_{n, x} v_{n, y}\left(X_{x}^{2}+X_{y}^{1}\right)+\mathrm{O}\left(n^{-1}\right) \\
= & I_{1}+I_{2}+I_{3}+I_{4}+I_{5}+I_{6}+\mathrm{O}\left(n^{-1}\right) .
\end{aligned}
$$

It follows from (5.14) that

$$
I_{3}+I_{4}+I_{5}=\mathrm{O}\left(n^{-1}\right) .
$$

Hence

$$
\begin{aligned}
\int_{P_{2}(0)} & \frac{1}{2}\left|D v_{n}\right|^{2} \operatorname{div}(X) \\
= & \int_{P_{2}(0)} \frac{1}{2}\left|D v_{n}\right|^{2}\left(X_{x}^{1}+X_{y}^{2}\right)+\sum_{j=3}^{m} \int_{P_{2}(0)} \frac{1}{2}\left|D v_{n}\right|^{2} X_{j}^{j} \\
& -\int_{P_{2}(0)}\left(\left|v_{n, x}\right|^{2} X_{x}^{1}+\left|v_{n, y}\right|^{2} X_{y}^{2}\right)+v_{n, x} v_{n, y}\left(X_{x}^{2}+X_{y}^{1}\right)+\mathrm{O}\left(n^{-1}\right) .
\end{aligned}
$$

By choosing $X=\left(0,0, X^{3}, \ldots, X^{m}\right)$, we then get

$$
\int_{P_{2}(0)} \frac{1}{2}\left|D v_{n}\right|^{2} \operatorname{div}(X)=\sum_{j=3}^{m} \int_{P_{2}(0)} \frac{1}{2}\left|D v_{n}\right|^{2} X_{j}^{j}+\mathrm{O}\left(n^{-1}\right)
$$

this, combined with (5.19) and (5.20), implies

$$
\begin{aligned}
\int_{P_{2}(0)} & \frac{1}{2}\left(\left|v_{n, x}\right|^{2}+\left|v_{n, y}\right|^{2}\right)\left(X_{x}^{1}+X_{y}^{2}\right) \\
= & \int_{P_{2}(0)}\left(\left|v_{n, x}\right|^{2} X_{x}^{1}+\left|v_{n, y}\right|^{2} X_{y}^{2}\right)+v_{n, x} v_{n, y}\left(X_{x}^{2}+X_{y}^{1}\right)+\mathrm{O}\left(n^{-1}\right) .
\end{aligned}
$$

In particular, one has

$$
\int_{P_{2}(0)}\left(\left|D_{x} v_{n}\right|^{2}-\left|D_{y} v_{n}\right|^{2}\right)\left(X_{y}^{2}-X_{x}^{1}\right)-2 \int_{P_{2}(0)} D_{x} v_{n} D_{y} v_{n}\left(X_{x}^{2}+X_{y}^{1}\right)=\mathrm{O}\left(n^{-1}\right) .
$$

Therefore, we get

$$
\int_{B_{2}^{m-2} \times(-4,4)}\left(\alpha(z, t)\left(X_{y}^{2}-X_{x}^{1}\right)-2 \beta(z, t)\left(X_{x}^{2}+X_{y}^{1}\right)=0\right.
$$

for any $X^{1}, X^{2} \in C_{0}^{1}\left(B_{2}^{m}\right)$. Now choosing $X^{1}(x, y, z)=x \phi(x, y, z)$ and $X^{2}=0$ for suitable cut-off function $\phi \in C_{0}^{1}\left(B_{2}^{m}\right)$, one can see

$$
\int_{B_{1}^{m-2} \times(-1,1)} \alpha(z, t) \mathrm{d} z \mathrm{~d} t=0 .
$$


Similarly, by choosing $X^{2}(x, y, z)=y \phi(x, y, z)$ and $X^{1}=0$, we obtain

$$
\int_{B_{1}^{m-2} \times(-1,1)} \beta(z, t) \mathrm{d} z \mathrm{~d} t=0 .
$$

This completes the proof of Lemma 5.8.

Proof of Lemma 5.7. - Note that Lemma 5.8 guarantees that for $\mathcal{P}^{m}$ a.e. $z_{0}=$ $\left(x_{0}, t_{0}\right) \in \Sigma$

$$
\int_{P_{r}\left(z_{0}\right)}\left|f_{n}\right|^{2}=\int_{P_{r}\left(z_{0}\right)}\left|g_{n}\right|^{2}=1+\mathrm{O}\left(r, n^{-1}\right), \quad \int_{P_{r}\left(z_{0}\right)} f_{n} \cdot g_{n}=\mathrm{O}\left(r, n^{-1}\right) .
$$

Here

$$
f_{n}=\frac{\sqrt{2} D_{x} u_{n}}{\left(\int_{P_{r}\left(z_{0}\right)}\left(\left|D_{x} u_{n}\right|^{2}+\left|D_{y} u_{n}\right|^{2}\right)\right)^{\frac{1}{2}}}, \quad g_{n}=\frac{\sqrt{2} D_{y} u_{n}}{\left(\int_{P_{r}\left(z_{0}\right)}\left(\left|D_{x} u_{n}\right|^{2}+\left|D_{y} u_{n}\right|^{2}\right)\right)^{\frac{1}{2}}} .
$$

Therefore, applying the Parseval's inequality, we have

$$
\lim _{r \downarrow 0} \lim _{n \rightarrow \infty} \int_{P_{r}\left(z_{0}\right)}\left|\partial_{t} u_{n}\right|^{2} \geqslant \lim _{r \downarrow 0} \lim _{n \rightarrow \infty}\left(\int_{P_{r}\left(z_{0}\right)} \partial_{t} u_{n} f_{n}\right)^{2}+\left(\int_{P_{r}\left(z_{0}\right)} \partial_{t} u_{n} g_{n}\right)^{2}
$$

substituting $f_{n}$ and $g_{n}$ into the inequality and using the fact that

$$
\lim _{r \downarrow 0} \lim _{n \rightarrow \infty} r^{-m} \int_{P_{r}\left(z_{0}\right)}\left|D_{z} u_{n}\right|^{2}=0
$$

we have

$$
\lim _{r \downarrow 0} \lim _{n \rightarrow \infty} \frac{\left(\int_{P_{r}\left(z_{0}\right)} \partial_{t} u_{n} D u_{n}\right)^{2}}{\int_{P_{r}\left(z_{0}\right)}\left|D u_{n}\right|^{2}} \leqslant \lim _{r \downarrow 0} \lim _{n \rightarrow \infty} \frac{1}{2} \int_{P_{r}\left(z_{0}\right)}\left|\partial_{t} u_{n}\right|^{2} .
$$

On the other hand, we know that for $\mathcal{P}^{m}$ a.e. $z_{0}=\left(x_{0}, t_{0}\right) \in \Sigma$,

$$
\left|H_{t_{0}}\left(x_{0}\right)\right|^{2} \mathrm{~d} \mu_{t}\left(z_{0}\right) \leqslant \lim _{r \downarrow 0} \lim _{n \rightarrow \infty} 2 \frac{\left|\int_{P_{r}\left(z_{0}\right)} \partial_{t} u_{n} D u_{n}\right|^{2}}{\int_{P_{r}\left(z_{0}\right)}\left|D u_{n}\right|^{2}} .
$$

Therefore, we have

$$
\begin{aligned}
\left|H_{t_{0}}\left(x_{0}\right)\right|^{2} \mathrm{~d} \mu\left(x_{0}, t_{0}\right) & \leqslant \lim _{r \downarrow 0} \lim _{n \downarrow \infty} \int_{P_{r}\left(z_{0}\right)}\left|\partial_{t} u_{n}\right|^{2} \mathrm{~d} x \mathrm{~d} t \\
& =\lim _{r \downarrow 0} \int_{P_{r}\left(z_{0}\right)}\left|\partial_{t} u\right|^{2}+\eta\left(P_{r}\left(z_{0}\right)\right)=\lim _{r \downarrow 0} \eta\left(P_{r}\left(z_{0}\right)\right) .
\end{aligned}
$$


This clearly implies

$$
\int_{\Sigma_{t}^{s}} \phi(x)\left|H_{t}(x)\right|^{2} \mathrm{~d} \mu_{t}(x) \mathrm{d} t \leqslant \int_{\Sigma_{t}^{s}} \phi(x) \mathrm{d} \eta(x, t), \quad \forall \phi \in C_{0}^{1}\left(\Omega, R_{+}\right) .
$$

Once Lemma 5.7 is proved, we see easily that Theorem 5.6 is proved as well. Now we introduce the notion of suitably weak solution to the heat equation of harmonic maps.

Definition 5.9. - A map $u \in H_{\mathrm{loc}}^{1}\left(\Omega \times R_{+}, N\right) \cap L^{\infty}\left(R_{+}, H^{1}(\Omega, N)\right)$ is a suitably weak solution to the heat equation of harmonic maps, if:

(1) It is a weak solution to the heat equation of harmonic maps (1.0).

(2) It satisfies the energy conservation law as follows. For any $0 \leqslant t_{1}<t_{2}<\infty$ and $\phi \in C_{0}^{1}\left(\Omega, R_{+}\right)$,

$$
\begin{aligned}
& \int_{\Omega} \frac{1}{2}|D u|^{2}\left(x, t_{2}\right) \phi(x)-\int_{\Omega} \frac{1}{2}|D u|^{2}\left(x, t_{1}\right) \phi(x) \\
&=-\int_{t_{1}}^{t_{2}} \int_{\Omega}\left(\left|\partial_{t} u\right|^{2}(x, t) \phi(x)+D \phi \partial_{t} u D u\right) .
\end{aligned}
$$

We would like to remark that it is easy to check that any smooth solution to (1.0) is a suitably weak solution.

A direct consequence of Theorem 5.6 is

COROLlARY 5.10. - Under the same assumptions as in Theorem 5.6. If, in addition, that the weak limiting map $u \in H_{\mathrm{loc}}^{1}\left(\Omega \times R_{+}, N\right)$ is a suitably weak solution to (1.0). Then, the defect measures $\left\{v_{t}\right\}_{t \geqslant 0}$ satisfies: for any $0 \leqslant s \leqslant t<\infty$ and $\phi \in C_{0}^{1}\left(\Omega, R_{+}\right)$,

$$
v_{t}(\phi)-v_{s}(\phi) \leqslant-\int_{s}^{t} \int_{\Sigma_{t}}\left(\phi(x)\left|H_{t}(x)\right|^{2}-\left\langle\left(T_{x} \Sigma_{t}\right)^{\perp} D \phi(x), H_{t}(x)\right\rangle\right) \mathrm{d} v_{t}(x) \mathrm{d} t .
$$

Now we want to show that (5.27) actually implies that $\left\{v_{t}\right\}_{t \geqslant 0}$ is a Brakke flow. First, let us recall the definition of Brakke flow given by Illmann [20], which is slightly stronger than the original definition by Brakke [8].

DEFINITION 5.11 ([8,20]). - Let $v$ be a Radon measure in $\Omega$ and $\phi \in C_{0}^{2}\left(\Omega, R_{+}\right)$, we set

$$
\mathcal{B}(v, \phi)=-\int_{\Omega}\left(\phi|H|^{2}-\left\langle\left(T_{x} v\right)^{\perp} D \phi, H\right\rangle\right) \mathrm{d} v
$$

provided that the following three conditions hold

(1) $v=\|V\|$ in $\{\phi>0\}$ for some $V \in R V_{m-2}(\Omega)$,

(2) $\delta V=H\|V\|$ in $\{\phi>0\}$,

(3) $H \in L_{\|V\|}^{2}\left(\{\phi>0\}, R^{m}\right)$.

Otherwise, we set $\mathcal{B}(\nu, \phi)=-\infty$. 
Definition $5.12([8,20])$. - Let $\left\{\mu_{t}\right\}_{t \geqslant 0}$ be Radon measures on $\Omega$. We say that $\left\{\mu_{t}\right\}_{t \geqslant 0}$ is a Brakke flow if

$$
\bar{D}_{t} \mu_{t}(\phi) \equiv \limsup _{s \rightarrow t} \frac{\mu_{s}(\phi)-\mu_{t}(\phi)}{s-t} \leqslant \mathcal{B}\left(\mu_{t}, \phi\right)
$$

for all $t \geqslant 0$ and $\phi \in C_{0}^{2}\left(\Omega, R_{+}\right)$.

THEOREM 5.13. - Under the same assumption as Theorem 5.6. If, in addition, that $u \in H_{\mathrm{loc}}^{1}\left(\Omega \times R_{+}, N\right)$ is a suitably weak solution to (1.0). Then $\left\{v_{t}\right\}_{t \geqslant 0}$ is a Brakke flow.

Proof. - First it follows from Section 4 and Lemma 5.2 that for $L^{1}$ a.e. $t \in R_{+}$, we have (a): $v_{t}=\left\|V_{t}\right\|$ for some $V_{t} \in R V_{m-2}(\Omega)$; (b): $\Theta^{m-2}\left(\left\|V_{t}\right\|, x\right) \geqslant \frac{\varepsilon_{0}^{2}}{4}$ for $H^{m-2}$ a.e. $x \in \Sigma_{t}$; (c): $\delta V_{t}=H_{t}\left\|V_{t}\right\|$ with $H_{t} \in L_{\left\|V_{t}\right\|}^{2}\left(\Omega, R^{m}\right)$; (d): $H_{t}(x) \perp T_{x}\left\|V_{t}\right\|$ for $H^{m-2}$ a.e. $x \in \Sigma_{t}$. Now we argue that (a)-(d) and (5.27) are sufficient to show (5.28) for $\left\{v_{t}\right\}_{t \geqslant 0}$. To see it, let us check the upper right derivative $\bar{D}_{+}$of $v_{t}$ for $t \geqslant 0$, the proof for lower right derivative is similar for $t>0$. Let

$$
L=\limsup _{s \downarrow t}-\frac{1}{s-t} \int_{t}^{s} \int_{\Omega}\left(\phi\left|H_{t}\right|^{2}-\left\langle D \phi, H_{t}\right\rangle\right) \mathrm{d} v_{t} \mathrm{~d} t .
$$

Note that (5.27) implies $L \geqslant \bar{D}_{+} v_{t}(\phi)$. If $L=-\infty$, then $\bar{D}_{+} v_{t}(\phi)=-\infty$ so that (5.28) holds automatically. Hence we assume that $L>-\infty$ and $\bar{D}_{+} v_{t}(\phi)>-\infty$. Let $s_{i} \downarrow t$ be such that

$$
\lim _{i \rightarrow \infty}-\frac{1}{s_{i}-t} \int_{t}^{s_{i}} \int_{\Omega}\left(\phi\left|H_{t}\right|^{2}-\left\langle D \phi, H_{t}\right\rangle\right) \mathrm{d} v_{t} \mathrm{~d} t=L
$$

and $t_{i} \in\left(t, s_{i}\right)$ be such that (a)-(d) hold at $t_{i}$ and

$$
\int_{\Omega}\left(\phi\left|H_{t_{i}}\right|^{2}-\left\langle\left(T_{x} \Sigma_{t_{i}}\right)^{\perp} D \phi, H_{t_{i}}\right\rangle\right) \mathrm{d} v_{t_{i}} \leqslant-L+\mathrm{O}\left(i^{-1}\right) .
$$

By the compactness theorem of Allard [6], we may assume that $V_{t_{i}} \rightarrow V$ in $\{\phi>0\} \times$ $G_{m-2, m}$ for some $V \in R V_{m-2}(\Omega)$. Moreover, by the result of Ilmanen [20] (cf. also Lemma 2.5 of Section 1), we know that $\|V\|=v_{t}$. There exists a $H \in L_{\|V\|}^{2}\left(\Omega, R^{m}\right)$ such that $\delta V=H\|V\|=H v_{t}$ and

$$
\begin{aligned}
& \int_{\Omega}\left(\phi|H|^{2}-\left\langle T_{x} \Sigma_{t}, H\right\rangle\right) \mathrm{d} v_{t} \\
& \quad \leqslant \liminf _{i \rightarrow \infty} \int_{\Omega}\left(\phi\left|H_{t_{i}}\right|^{2}-\left\langle\left(T_{x} \Sigma_{t_{i}}\right)^{\perp} D \phi, H_{t_{i}}\right\rangle\right) \mathrm{d} v_{t_{i}}=-L .
\end{aligned}
$$


Therefore

$$
\bar{D}_{+} v_{t}(\phi) \leqslant L \leqslant-\int_{\Omega}\left(\phi|H|^{2}-\left\langle T_{x} \Sigma_{t}, H\right\rangle\right) \mathrm{d} v_{t}=\mathcal{B}\left(v_{t}, \phi\right) .
$$

We end this section with the following remark.

Remark 5.14. - (1) It follows from Proposition 5.3 of Ambrosio and Soner [2] that the Brakke flow is also a distance solution to the mean curvature flow. Therefore, under the condition that $u$ is a suitable weak solution, Theorem 5.13 implies that $\left\{v_{t}\right\}_{t \geqslant 0}$ is a distance solution to the mean curvature flow.

(2) Under the assumption that $u$ is a suitable weak solution. If $v_{0}=\alpha H^{m-2} \mathrm{~L} \Gamma_{0}$ for some $\alpha>0$ and a closed $(m-2)$-dimensional Riemannian manifold $\Gamma_{0}$. Let $\left\{\Gamma_{t}\right\}_{t \in[0, T)}$ is the smooth mean curvature flow. Then there exists a nonincreasing function $\alpha:[0, T) \rightarrow[0, \alpha]$ such that $v_{t}=\alpha(t) H^{m-2} \mathrm{~L} \Gamma_{t}$ for $t \in[0, T)$ (see Proposition 4.5 of [1], and also $[25,22])$.

\section{Energy quantization of the energy density function}

Throughout this section, we assume that $N=S^{k-1} \subset R^{k}$ and $m \geqslant 3$. We will show that, for $\mathcal{P}^{m}$ a.e. $z_{0}=\left(x_{0}, t_{0}\right) \in \Sigma$, the density function $\Theta^{m-2}\left(\left\|V_{t_{0}}\right\|, x_{0}\right)$ is the finite sum of energies of harmonic $S^{2}$ 's (i.e., nontrivial harmonic maps from $S^{2}$ ). In the static case, this type of quantization result was first obtained by Lin and Rivieré [27] for stationary harmonic maps, and then Lin and Wang [32] for critical points for Ginzburg-Landau functionals. Our results here can be viewed as the parabolic extension of that of [27, 32]. For $m=2$, this type of quantization result is called as energy identity or bubbling phenomena by people (see, [34] and [32] references therein). Let's consider the heat flow of the Ginzburg-Landau functional here, the corresponding result for the heat flow of harmonic maps is treated in Section 7.

The main theorem of this section is

THEOREM 6.1. - For $\mathcal{P}^{m}$ a.e. $z_{0}=\left(x_{0}, t_{0}\right) \in \Sigma$,

$$
\Theta^{m-2}\left(\left\|V_{t_{0}}\right\|, x_{0}\right)=\sum_{i=1}^{l_{z_{0}}} E\left(\phi_{i}, S^{2}\right)
$$

for some $1 \leqslant l_{z_{0}}<\infty$, here $\phi_{i}: S^{2} \rightarrow S^{k-1}\left(1 \leqslant i \leqslant l_{z_{0}}\right)$ are nontrivial harmonic maps.

Proof. - Let us first list all the necessary facts needed, which can be found from Sections 3, 5, and [31]. The following properties hold: For $L^{1}$ a.e $t_{0} \in R_{+}$,

$$
\begin{gathered}
\lim _{n \rightarrow \infty} \int_{\Omega}\left|\partial_{t} u_{n}\right|^{2}\left(x, t_{0}\right) \mathrm{d} x<\infty, \\
\lim _{r \downarrow 0} \lim _{n \rightarrow \infty} r^{2-m} \int_{B_{r}(x)}\left|\partial_{t} u_{n}\right|^{2}<\infty, \quad \text { for } H^{m-2} \text { a.e. } x \in \Sigma_{t_{0}}
\end{gathered}
$$


and, for $\mathcal{P}^{m}$ a.e. $z_{0}=\left(x_{0}, t_{0}\right) \in \Sigma$,

$$
\begin{gathered}
\varepsilon_{0}^{2} \leqslant \Theta^{m-2}\left(\left\|V_{t_{0}}\right\|, x_{0}\right)<\infty, \\
\lim _{r \downarrow 0} \lim _{n \rightarrow \infty} r^{2-m} \int_{P_{r}\left(z_{0}\right)}\left|\partial_{t} u_{n}\right|^{2}=0,
\end{gathered}
$$

$\Theta^{m}\left(\mu, z_{0}\right)$ is $\mathcal{P}^{m}$ approximately continuous at $z_{0}$,

$$
\begin{gathered}
\lim _{r \downarrow 0} \lim _{n \rightarrow \infty} r^{-m} \int_{P_{r}\left(z_{0}\right)}\left|D_{T} u_{n}\right|^{2}=0, \quad \forall T \in T_{x_{0}} \Sigma_{t_{0}}, \\
\lim _{r \downarrow 0} r^{-m} \int_{P_{r}\left(z_{0}\right)}|D u|^{2}+r^{2}\left|\partial_{t} u\right|^{2}=0,
\end{gathered}
$$

and, for $L^{1}$ a.e. $t_{0} \in R_{+}$, we have, for $H^{m-2}$ a.e. $x_{0} \in \Sigma_{t_{0}}$,

$$
\Theta^{m-2}\left(\left\|V_{t_{0}}\right\|, x_{0}\right) \text { is } H^{m-2} \text { approximately continuous at } x_{0} .
$$

Let us now pick up a $z_{0}=\left(x_{0}, t_{0}\right) \in \Sigma$ such that (6.2)-(6.9) all hold. Moreover, we may assume that $T_{x_{0}} \Sigma_{t_{0}}=\{(0,0)\} \times R^{m-2}=\left\{(0,0, Y): Y \in R^{m-2}\right\}$, and write $x=(X, Y) \in$ $R^{2} \times R^{m-2}$ for $x \in R^{m}$. For any $r_{n} \downarrow 0$, define the rescaling maps $v_{n}: P_{2}(0) \rightarrow R^{k}$ by letting $v_{n}(x, t)=u_{n}\left(x_{0}+r_{n} x, t_{0}+r_{n}^{2} t\right)$. Then, we have,

$$
\begin{gathered}
\lim _{n \rightarrow \infty} \int_{P_{1}(0)}\left|D_{Y} v_{n}\right|^{2}+\left|\partial_{t} v_{n}\right|^{2}=0, \\
v_{n} \rightarrow \text { constant weakly in } H^{1}\left(P_{2}(0), R^{k}\right), \\
e\left(v_{n}\right)(X, Y, t) \mathrm{d} X \mathrm{~d} Y \mathrm{~d} t \rightarrow \bar{v}_{t} \mathrm{~d} t
\end{gathered}
$$

as convergence of Radon measures on $P_{2}(0)$. Similar to [31], we have

Claim 6.2. $-\bar{v}_{t} \mathrm{~d} t=\Theta^{m-2}\left(\left\|V_{t_{0}}\right\|, x_{0}\right) H^{m-2} \mathrm{~L} R^{m-2} \times L^{1} \mathrm{~L} R$, on $P_{1}(0)$.

To see this, let $\phi \in C_{0}^{1}\left(B_{1}^{2}, R_{+}\right)$and define $f_{n}, g_{n}, h_{n}: R^{m-2} \times R \rightarrow R_{+}$by

$$
f_{n}(Y, t)=\int_{B_{1}^{2}} e\left(v_{n}\right)(X, Y, t) \phi(X) \mathrm{d} X, \quad g_{n}(Y, t)=\int_{B_{1}^{2}}\left|\partial_{t} v_{n}\right|^{2}(X, Y, t) \mathrm{d} X
$$

and

$$
h_{n}(Y, t)=\int_{B_{1}^{2}}\left|D_{Y} v_{n}\right|^{2}(X, Y, t) \mathrm{d} X
$$


Then (6.10) implies

$$
\lim _{n \rightarrow \infty} \int_{B_{1}^{m-2} \times(-1,1)} g_{n}(Y, t)+h_{n}(Y, t) \mathrm{d} Y \mathrm{~d} t=0 .
$$

For $1 \leqslant j \leqslant m-2, Y_{j}$ and $t$-derivative of $f_{n}$ are as follows.

$$
\begin{aligned}
\frac{\mathrm{d}}{\mathrm{d} Y_{j}} f_{n}(Y, t)= & \int_{B_{1}^{2}}\left(D_{X} v_{n} D_{X Y_{j}}^{2} v_{n}-\frac{1}{\varepsilon_{n}^{2}} f\left(v_{n}\right) D_{Y_{j}} v_{n}\right) \phi+\int_{B_{1}^{2}} D_{Y_{i}} v_{n} D_{Y_{i} Y_{j}}^{2} v_{n} \phi \\
= & -\int_{B_{1}^{2}} \phi\left(\Delta_{X} v_{n}+\frac{1}{\varepsilon_{n}^{2}} f\left(v_{n}\right)\right) D_{Y_{j}} v_{n} \\
& -\int_{B_{1}^{2}} D_{X} \phi D_{X} v_{n} D_{Y_{j}} v_{n}+\int_{B_{1}^{2}} D_{Y_{i}} v_{n} D_{Y_{i} Y_{j}}^{2} v_{n} \phi \\
= & -\int_{B_{1}^{2}} \phi \partial_{t} v_{n} D_{Y_{j}} v_{n}-\int_{B_{1}^{2}} D_{X} \phi D_{X} v_{n} D_{Y_{j}} v_{n}+\frac{\mathrm{d}}{\mathrm{d} Y_{i}} \int_{B_{1}^{2}} \phi D_{Y_{i}} v_{n} D_{Y_{j}} v_{n} \\
= & f_{n}^{1, j}+\operatorname{div}_{(Y, t)} f_{n}^{2, j} .
\end{aligned}
$$

Here

$$
f_{n}^{1, j}(Y, t)=-\int_{B_{1}^{2}}\left(D_{X} \phi D_{X} v_{n} D_{Y_{j}} v_{n}+\phi \partial_{t} v_{n} D_{Y_{j}} v_{n}\right)
$$

and

$$
\begin{aligned}
f_{n}^{2, j}(Y, t)= & \left(\int_{B_{1}^{2}} \phi D_{Y_{1}} v_{n} D_{Y_{j}} v_{n}, \ldots, \int_{B_{1}^{2}} \phi D_{Y_{m-2}} v_{n} D_{Y_{j}} v_{n}, 0\right) . \\
\frac{\mathrm{d}}{\mathrm{d} t} g_{n}(Y, t)= & -\int_{B_{1}^{2}}\left(\Delta_{X} v_{n}+\frac{1}{\varepsilon_{n}^{2}} f\left(v_{n}\right)\right) \partial_{t} v_{n} \phi \\
& -\int_{B_{1}^{2}} D_{X} \phi D_{X} v_{n} \partial_{t} v_{n}+\int_{B_{2}^{2}} \phi D_{Y_{i}} v_{n} D_{Y_{i}}\left(\partial_{t} v_{n}\right) \\
= & -\int_{B_{1}^{2}}\left|\partial_{t} v_{n}\right|^{2} \phi-\int_{B_{1}^{2}} D_{X} v_{n} D_{X} \phi \partial_{t} v_{n}+\frac{\mathrm{d}}{\mathrm{d} Y_{i}} \int_{B_{1}^{2}} \phi D_{Y_{i}} v_{n} \partial_{t} v_{n} \\
= & g_{n}^{1}+\operatorname{div}_{(Y, t)} g_{n}^{2} .
\end{aligned}
$$

Here

$$
g_{n}^{1}(Y, t)=-\int_{B_{1}^{2}}\left(\left|\partial_{t} v_{n}\right|^{2} \phi+D_{X} v_{n} D_{X} \phi \partial_{t} v_{n}\right)
$$


and

$$
g_{n}^{2}(Y, t)=\left(\int_{B_{1}^{2}} \phi D_{Y_{1}} v_{n} \partial_{t} v_{n}, \ldots, \int_{B_{1}^{2}} \phi D_{Y_{m-2}} v_{n} \partial_{t} v_{n}, 0\right) .
$$

Note that (6.10) implies

$$
\lim _{n \rightarrow \infty} \sum_{i=1}^{2}\left(\left\|f_{n}^{i}\right\|_{L^{1}\left(B_{1}^{m-2} \times(-1,1)\right)}+\left\|g_{n}^{i}\right\|_{L^{1}\left(B_{1}^{m-2} \times(-1,1)\right)}\right)=0 .
$$

Based on (6.12), (6.13), and (6.14), we can apply the Allard's strong constancy Lemma [5] as in [23] or [31] to conclude the Claim 6.2. Moreover, one has

$$
\lim _{n \rightarrow \infty}\left\|f_{n}(Y, t)-\Theta^{m-2}\left(\left\|V_{t_{0}}\right\|, x_{0}\right)\right\|_{L^{1}\left(B_{1}^{m-2} \times(-1,1)\right)}=0 .
$$

Therefore, for any $\delta>0$, there exists $E_{\delta} \subset B_{1}^{m-2} \times(-1,1)$ with $\left|E_{\delta}\right| \geqslant 1-\delta$ such that

$$
\lim _{n \rightarrow \infty} \sup _{(Y, t) \in E_{\delta}}\left|f_{n}(Y, t)-\Theta^{m-2}\left(\left\|V_{t_{0}}\right\|, x_{0}\right)\right|=0
$$

In order to prove that $\Theta^{m-2}\left(\left\|V_{t_{0}}\right\|, x_{0}\right)$ is the sum of energies of finitely many harmonic $S^{2}$ 's, it suffices to prove that $f_{n}(Y, t)$ converges to the sum of energies of finitely many harmonic $S^{2}$ 's, for $(Y, t) \in E_{\delta}$. Now we define the local Hardy-Littlewood maximal function for a function $f \in L^{1}\left(B_{1}^{m-2} \times(-1,1)\right)$, with respect to the parabolic metric in $R^{m-2} \times R$, as follows

$$
M(f)(Y, t)=\sup \left\{r^{-m} \int_{P_{r}(Y, t)}(f)(z, s) \mathrm{d} y \mathrm{~d} s, P_{r}(Y, t) \subset B_{1}^{m-2} \times(-1,1)\right\} .
$$

Then the weak $(1,1)$ estimates implies that there exists $F_{\delta}^{n} \subset B_{1}^{m-2} \times(-1,1)$, with $\left|F_{\delta}^{n}\right| \geqslant 1-\delta$, such that

$$
\begin{aligned}
\lim _{n \rightarrow \infty} M\left(g_{n}+h_{n}\right)(Y, t) & =0, \quad \forall(Y, t) \in F_{\delta}^{n}, \\
& \lim _{n \rightarrow \infty} M\left(f_{n}\right)(Y, t) \leqslant C \Theta^{m-2}\left(\left\|V_{t_{0}}\right\|, x_{0}\right), \quad \forall(Y, t) \in F_{\delta}^{n}, \\
\lim _{n \rightarrow \infty} M\left(p_{n}\right)(Y, t) & =0, \quad \forall(Y, t) \in F_{\delta}^{n},
\end{aligned}
$$

where

$$
p_{n}(Y, t)=\int_{B_{1}^{2}} \frac{1}{\bar{\varepsilon}_{n}^{2}} F\left(v_{n}\right)(X, Y, t) \mathrm{d} X
$$

here $\bar{\varepsilon}_{n}=\frac{\varepsilon_{n}}{r_{n}} \rightarrow 0$. Now we try to prove that for any $(Y, t) \in E_{\delta} \cap F_{\delta}^{n}$

$$
\lim _{n \rightarrow \infty} f_{n}(Y, t)=\sum_{j=1}^{l} E\left(\phi_{j}, S^{2}\right)
$$


for some $1 \leqslant l<\infty$, here $\phi_{j}: S^{2} \rightarrow S^{k-1}(1 \leqslant j \leqslant l)$ are nontrivial harmonic maps.

Step 1. First Bubble.

This step has been carried out in [31]. Here we give a slightly different proof. For any given $\left(Y_{n}, t_{n}\right) \in E_{\delta} \cap F_{\delta}^{n}$, let $X_{n} \in B_{\frac{1}{2}}^{2}$ and $\delta_{n}>0$ be such that

$$
\int_{B_{\delta_{n}}^{2}} e\left(v_{n}\right)\left(X, Y_{n}, t_{n}\right) \mathrm{d} X=\frac{\varepsilon_{0}^{2}}{C(m)}=\max \left\{\int_{B_{\delta_{n}}^{2}(\bar{X})} e\left(v_{n}\right)\left(X, Y_{n}, t_{n}\right) \mathrm{d} X: \bar{X} \in B_{\frac{1}{2}}^{2}\right\}
$$

Here $\varepsilon_{0}>0$ is given by Lemma 2.3 and $C(m)>0$ is a large constant to be chosen. It is not difficult to see that $X_{n} \rightarrow 0$ and $\delta_{n} \rightarrow 0$ (cf. [23] and [31]). Moreover, as in [23] and [31], we can apply (6.12)-(6.14) to get, for any $X \in B_{\frac{1}{2}}^{2}$,

$$
\left(2 \delta_{n}\right)^{-m} \int_{B_{2 \delta_{n}}^{2}(X) \times B_{2 \delta_{n}}^{m-2}\left(Y_{n}\right) \times\left(t_{n}-4 \delta_{n}^{2}, t_{n}+4 \delta_{n}^{2}\right)} e\left(v_{n}\right)(X, Y, t) \mathrm{d} X \mathrm{~d} Y \mathrm{~d} t \leqslant \varepsilon_{0}^{2}
$$

and

$$
\delta_{n}^{-m} \int_{B_{\delta_{n}}^{2}(X) \times B_{\delta_{n}}^{m-2}\left(Y_{n}\right) \times\left(t_{n}-\delta_{n}^{2}, t_{n}+\delta_{n}^{2}\right)} e\left(v_{n}\right)(X, Y, t) \mathrm{d} X \mathrm{~d} Y \mathrm{~d} t \geqslant \frac{\varepsilon_{0}^{2}}{2} .
$$

We let

$$
w_{n}(X, Y, t)=v_{n}\left(X_{n}+\delta_{n} X, Y_{n}+\delta_{n} Y, t_{n}+\delta_{n}^{2} t\right) .
$$

Then Lemma 2.3 implies

$$
w_{n} \rightarrow w \quad \text { in } C_{\text {loc }}^{1}\left(R^{2} \times B_{2}^{m-2} \times(-4,4), R^{k}\right) .
$$

Moreover, (6.14) implies that $\partial_{t} w=D_{Y} w=0$ so that $w(X, Y, t)=w(X): R^{2} \rightarrow S^{k-1}$ is a harmonic map with positive and finite energy, which can be lifted to a nontrivial harmonic map from $S^{2}$ to $S^{k-1}$, named as $\phi_{1}$. By repeating all the possible blowing-up at different points and scales, we can get

$$
\Theta^{m-2}\left(\left\|V_{t_{0}}\right\|, x_{0}\right)=\lim _{n \rightarrow \infty} f_{n}\left(Y_{n}, t_{n}\right) \geqslant \sum_{j=1}^{l} E\left(\phi_{j}, S^{2}\right)
$$

for some $l=l_{z_{0}} \leqslant \Theta^{m-2}\left(\left\|V_{t_{0}}\right\|, x_{0}\right) / \varepsilon_{0}^{2}$, and some nontrivial harmonic maps $\phi_{j}: S^{2} \rightarrow$ $S^{k-1}(1 \leqslant j \leqslant l)$.

Step 2. (6.24) is an equality.

To achieve this, it suffices to show that there is no energy concentration over the neck regions between two bubbles at the same point. This step is very similar to that of [32]. The idea is to use the interpolation between $L^{2,1}$ and $L^{2, \infty}$ norms of $D v_{n}$ over the neck region, which has been recently explored by Lin and Riviere [27] in the context of stationary harmonic maps in higher dimensions, and $[28,32]$ in the context of critical points of Ginzburg-Landau functionals. For completeness, we sketch it here. First, we 
observe that by an induction argument on $l$ it suffices to show that (6.24) is an equality for $l=1$ (cf. [14] for $m=2$ and [27,32] for $m \geqslant 3$ for the induction argument).

Claim 6.3. - For any $\varepsilon>0$ and sufficiently large $R>0$, we have

$$
\int_{B_{2 r}^{2}\left(X_{n}\right) \backslash B_{r}^{2}\left(X_{n}\right)} e\left(v_{n}\right)\left(X, Y_{n}, t_{n}\right) \mathrm{d} X \leqslant \varepsilon^{2}, \quad \forall R \delta_{n}<r<\frac{1}{2} .
$$

For, otherwise, one can argue exactly as in [32] to conclude that one can rescale $v_{n}$ suitably to get a second bubble, which would contradict with $l=1$.

Now one can apply the Allard's strong constancy lemma (cf. [5] and [32]) and Lemma 2.3 to conclude that

$$
e\left(v_{n}\right)(X, Y, t) \leqslant \frac{C \varepsilon^{2}}{\left|X-X_{n}\right|^{2}+\left|Y-Y_{n}\right|^{2}+\left|t-t_{n}\right|^{2}}
$$

for $2 R \delta_{n} \leqslant\left|X-X_{n}\right| \leqslant \frac{1}{4},\left|Y-Y_{n}\right| \leqslant \frac{\left|X-X_{n}\right|}{2},\left|t-t_{n}\right| \leqslant \frac{\left|X-X_{n}\right|^{2}}{4}$. In particular, we have

$$
e\left(v_{n}\right)(X, Y, t) \leqslant \frac{C \varepsilon^{2}}{\left|X-X_{n}\right|^{2}}
$$

for $2 R \delta_{n} \leqslant\left|X-X_{n}\right| \leqslant \frac{1}{4},\left|Y-Y_{n}\right| \leqslant R \delta_{n},\left|t-t_{n}\right| \leqslant R^{2} \delta_{n}^{2}$. Hence, if we let $w_{n}(X, Y, t)=$ $v_{n}\left(X_{n}+\delta_{n} X, Y_{n}+\delta_{n} Y, t_{n}+\delta_{n}^{2} t\right)$, then we have

$$
e\left(w_{n}\right)(X, Y, t) \leqslant \frac{C \varepsilon^{2}}{|X|^{2}}, \quad \forall 2 R \leqslant|X| \leqslant \frac{1}{4 \delta_{n}},|Y| \leqslant R,|t| \leqslant R^{2} .
$$

This implies that $D w_{n}(\cdot, Y, t) \in L^{2, \infty}\left(B_{\left(4 \delta_{n}\right)^{-1}}^{2} \backslash B_{2 R}^{2}\right)$ for any $(Y, t) \in B_{R}^{m-2} \times\left(-R^{2}, R^{2}\right)$, and

$$
\sup _{(Y, t) \in B_{R}^{m-2} \times\left(-R^{2}, R^{2}\right)}\left\|D w_{n}(\cdot, Y, t)\right\|_{L^{2, \infty}\left(B_{\left(4 \delta_{n}\right)-1}^{2} \backslash B_{2 R}^{2}\right)} \leqslant C \varepsilon .
$$

Here $L^{2, \infty}$ denotes the Lorentz space with index $(2, \infty)$ (see Ziemer [38] for the definition). Now we try to estimate the $L^{2,1}$ norm of $D \frac{w_{n}}{\left|w_{n}\right|}(\cdot, Y, t)$ over $B_{\left(4 \delta_{n}\right)^{-1}}^{2}$.

CLAIM 6.4. - For $\mathcal{P}^{m}$ a.e. $(Y, t) \in B_{R}^{m-2} \times\left(-R^{2}, R^{2}\right), D \frac{w_{n}}{\left|w_{n}\right|}(\cdot, Y, t) \in L^{2,1}\left(B_{\left(4 \delta_{n}\right)^{-1}}^{2}\right)$. Moreover,

$$
\begin{aligned}
& \int_{B_{R}^{m-2} \times\left(-R^{2}, R^{2}\right)}\left\|D \frac{w_{n}}{\left|w_{n}\right|}(\cdot, Y, t)\right\|_{L^{2,1}\left(B_{\left(4 \delta_{n}\right)^{-1}}^{2}\right)} \mathrm{d} Y \mathrm{~d} t \\
& \leqslant C \delta_{n}^{-m} \int_{B_{\frac{1}{2}}^{2}\left(X_{n}\right) \times B_{R \delta_{n}}^{m-2}\left(Y_{n}\right) \times\left(t_{n}-R^{2} \delta_{n}^{2}, t_{n}+R^{2} \delta_{n}^{2}\right)}\left|D v_{n}\right|^{2}+\left|\partial_{t} v_{n}\right|^{2} \leqslant C .
\end{aligned}
$$

Proof. - It is very similar to the proof of Theorem B in [32]. Here we only sketch the outline. For any $t \in\left(-R^{2}, R^{2}\right)$, denote $\omega_{n}(X, Y)=w_{n}(X, Y, t): B_{\left(2 \delta_{n}\right)^{-1}}^{2} \times B_{2 R}^{m-2} \rightarrow R^{k}$. 
Then we have

$$
\Delta \omega_{n}+\frac{1}{\varepsilon_{n}^{2}}\left(1-\left|\omega_{n}\right|^{2}\right) \omega_{n}=l_{n}
$$

where $l_{n}(X, Y)=\partial_{t} w_{n}(X, Y, t)$.

For $1 \leqslant i, j \leqslant k$, let $\alpha_{n}^{i j}$ be the 1 -forms defined by $\alpha_{n}^{i j}=\mathrm{d} \omega_{n}^{i} \omega_{n}^{j}-\omega_{n}^{i} \mathrm{~d} \omega_{n}^{j}$. Then

$$
\begin{gathered}
\mathrm{d}^{*} \alpha_{n}^{i j}=\Delta \omega_{n}^{i} \omega_{n}^{j}-\Delta \omega_{n}^{j} \omega_{n}^{i} \\
=l_{n}^{i} \omega_{n}^{j}-l_{n}^{j} \omega_{n}^{i} \equiv H_{n}^{i j}, \\
\Delta \alpha_{n}^{i j}=\mathrm{d} H_{n}^{i j}+2 \mathrm{~d}^{*}\left(\mathrm{~d} \omega_{n}^{i} \wedge \mathrm{d} \omega_{n}^{j}\right) .
\end{gathered}
$$

Now, let $\tilde{\omega}_{n}: R^{m} \rightarrow R^{k}$ be an extension of $\omega_{n}$ such that

$$
\left\|D \tilde{\omega}_{n}\right\|_{L^{2}\left(R^{m}\right)} \leqslant C\left\|D \omega_{n}\right\|_{L^{2}\left(B_{\left(2 \delta_{n}\right)^{-1}}^{2} \times B_{2 R}^{m-2}\right)}
$$

and $\bar{H}_{n}^{i j}: R^{m} \rightarrow R$ be an extension of $H_{n}^{i j}$ such that $\bar{H}_{n}^{i j}=0$ outside $B_{\left(2 \delta_{n}\right)^{-1}}^{2} \times B_{2 R}^{m-2}$. Let $F_{n}^{i j} \in H^{1}\left(R^{m}, \wedge^{2}\left(R^{m}\right)\right)$ solve

$$
\Delta F_{n}^{i j}=2 \mathrm{~d} \tilde{\omega}_{n}^{i} \wedge \mathrm{d} \tilde{\omega}_{n}^{j}
$$

Then, we know from [32] that $F_{n}^{i j} \in W^{2,1}\left(R^{m}, \wedge^{2}\left(R^{m}\right)\right)$ and

$$
\begin{aligned}
\left\|D^{2} F_{n}^{i j}\right\|_{L^{1}\left(R^{m}\right)} & \leqslant C\left\|\mathrm{~d} \tilde{\omega}_{n}^{i} \wedge \mathrm{d} \tilde{\omega}_{n}^{j}\right\|_{\mathcal{H}^{1}\left(R^{m}\right)} \\
& \leqslant C\left\|D \tilde{\omega}_{n}\right\|_{L^{2}\left(R^{m}\right)}^{2} \\
& \leqslant C\left\|D \omega_{n}\right\|_{L^{2}\left(B_{\left(2 \delta_{n}\right)^{-1}}^{2} \times B_{2 R}^{m-2}\right)}^{2},
\end{aligned}
$$

where $\mathcal{H}^{1}$ denotes the Hardy space of $R^{m}$. Let $G_{n}^{i j} \in H^{1}\left(R^{m}, R\right)$ solve

$$
\Delta G_{n}^{i j}=\bar{H}_{n}^{i j}
$$

Then, we have that $D^{2} G_{n}^{i j} \in L^{2}\left(R^{m}\right)$ and

$$
\left\|D^{2} G_{n}^{i j}\right\|_{L^{2}\left(R^{m}\right)} \leqslant C\left\|\bar{H}_{n}^{i j}\right\|_{L^{2}\left(R^{m}\right)} \leqslant C\left\|\partial_{t} w_{n}\right\|_{L^{2}\left(B_{\left(2 \delta_{n}\right)-1}^{2} \times B_{2 R}^{m-2}\right)} .
$$

In particular, we have, by the Hölder inequality,

$$
\begin{aligned}
\left\|D^{2} G_{n}^{i j}\right\|_{L^{1}\left(B_{\left(2 \delta_{n}\right)^{-1}}^{2} \times B_{2 R}^{m-2}\right)} & \leqslant C\left\|D^{2} G_{n}^{i j}\right\|_{L^{2}\left(B_{\left(2 \delta_{n}\right)^{-1}}^{2} \times B_{2 R}^{m-2}\right)}\left(R^{m-2} \delta_{n}^{-2}\right)^{\frac{1}{2}} \\
& \leqslant C\left(R^{m-2} \delta_{n}^{-2}\right)^{\frac{1}{2}}\left\|\partial_{t} w_{n}\right\|_{L^{2}\left(B_{\left(2 \delta_{n}\right)^{-1}}^{2} \times B_{2 R}^{m-2}\right)^{-}}
\end{aligned}
$$

Note that

$$
\alpha_{n}^{i j}=\mathrm{d} G_{n}^{i j}+2 \mathrm{~d}^{*}\left(F_{n}^{i j}\right)+K_{n}^{i j},
$$


where $K_{n}^{i j}$ is a harmonic 1-form with $j^{*}\left(\alpha_{n}^{i j}-2 \mathrm{~d}^{*} F_{n}^{i j}-\mathrm{d} G_{n}^{i j}\right)=0$, and $j: \partial\left(B_{\left(2 \delta_{n}\right)^{-1}}^{2} \times\right.$ $\left.B_{\frac{3 R}{2}}^{m-2}\right) \rightarrow R^{m}$ denotes the inclusion map. By choosing $R>0$ suitably and using the Fubini's theorem, we may assume that

$$
\begin{gathered}
\left\|\alpha_{n}^{i j}\right\|_{L^{1}\left(\partial\left(B_{\left(2 \delta_{n}\right)^{-1}}^{2} \times B_{\frac{3 R}{2}}^{m-2}\right)\right)} \leqslant C R^{-1}\left\|D \omega_{n}\right\|_{L^{1}\left(B_{\delta_{n}^{-1}}^{2} \times B_{2 R}^{m-2}\right)}, \\
\left\|\left|D^{2} G_{n}^{i j}\right|+\left|D^{2} F_{n}^{i j}\right|\right\|_{L^{1}\left(\partial\left(B_{\left(2 \delta_{n}\right)^{-1}}^{2} \times B_{\frac{3 R}{2}}^{m-2}\right)\right)} \\
\leqslant C R^{-1}\left\|\left|D^{2} G_{n}^{i j}\right|+\left|D^{2} F_{n}^{i j}\right|\right\|_{L^{1}\left(\left(B_{\delta_{n}^{-1}}^{2} \times B_{2 R}^{m-2}\right)\right)} .
\end{gathered}
$$

Therefore, by the well-known estimate on harmonic functions, we have

$$
\begin{aligned}
& \left\|D K_{n}^{i j}\right\|_{L^{1}\left(B_{\left(4 \delta_{n}\right)-1}^{2} \times B_{R}^{m-2}\right)} \\
& \leqslant C\left(R^{m-2} \delta_{n}^{-2}\right)^{\frac{1}{2}}\left(\int_{B_{\delta_{n}^{2}}^{2} \times B_{R}^{m-2}}\left(\left|D w_{n}\right|^{2}+\left|\partial_{t} w_{n}\right|^{2}\right) \mathrm{d} X \mathrm{~d} Y\right)^{\frac{1}{2}} .
\end{aligned}
$$

Hence it follows from the embedding result, $W^{1,1}\left(R^{2}\right) \subset L^{2,1}\left(R^{2}\right)$ (cf. Heléin [19]), that we have, for $H^{m-2}$ a.e. $Y \in B_{R}^{m-2}$, that $\alpha_{n}^{i j}(\cdot, Y) \in L^{2,1}\left(B_{\left(4 \delta_{n}\right)^{-1}}^{2}\right)$ and

$$
\begin{aligned}
\left\|\alpha_{n}^{i j}(\cdot, Y)\right\|_{L^{2,1}\left(B_{(4 \delta)^{-1}}^{2}\right)} & \leqslant C\left\|D \alpha_{n}^{i j}\right\|_{W^{1,1}\left(B_{\left(4 \delta_{n}\right)^{-1}}^{2}\right)} \\
& \leqslant C\left\|\left|D^{2} G_{n}^{i j}\right|+\left|D^{2} F_{n}^{i j}\right|+\left|D K_{n}^{i j}\right|\right\|_{L^{1}\left(B_{\left(4 \delta_{n}\right)^{-1}}^{2}\right)} .
\end{aligned}
$$

Therefore

$$
\begin{aligned}
& \int_{B_{R}^{m-2}}\left\|\alpha_{n}^{i j}(\cdot, Y)\right\|_{L^{2,1}\left(B_{\left(4 \delta_{n}\right)^{-1}}^{2}\right)} \mathrm{d} Y \\
& \leqslant C R^{m-2} \delta_{n}^{-2} \int_{\substack{B_{\delta_{n}^{-1}}^{2} \times B_{2 R}^{m-2} \\
\leqslant}}\left(\left|D w_{n}\right|^{2}+\left|\partial_{t} w_{n}\right|^{2}\right)(X, Y, t) \mathrm{d} X \mathrm{~d} Y .
\end{aligned}
$$

Hence, by the duality between $L^{2,1}$ and $L^{2, \infty}$ and (6.25), we obtain

$$
\begin{aligned}
& \quad \int_{\left(B_{\left(4 \delta_{n}\right)^{-1}}^{2} \backslash B_{2 R}^{2}\right) \times B_{R}^{m-2}}\left|\alpha_{n}^{i j}\right|^{2}(X, Y) \mathrm{d} X \mathrm{~d} Y \\
& \leqslant \int_{B_{R}^{m-2}}\left\|\alpha_{n}^{i j}(\cdot, Y)\right\|_{L^{2,1}\left(B_{\left(4 \delta_{n}\right)^{-1}}^{2}\right)}\left\|\alpha_{n}^{i j}(\cdot, Y)\right\|_{L^{2, \infty}\left(B_{\left(4 \delta_{n}\right)^{-1}}^{2} \backslash B_{2 R}^{2}\right)} \mathrm{d} Y \\
& \leqslant \sup _{Y \in B_{R}^{m-2}}\left\|\alpha_{n}^{i j}(\cdot, Y)\right\|_{L^{2, \infty}\left(B_{\left(4 \delta_{n}\right)^{-1}}^{2} \backslash B_{2 R}^{2}\right)} \int_{B_{R}^{m-2}}\left\|\alpha_{n}^{i j}(\cdot, Y)\right\|_{L^{2,1}\left(B_{\left(4 \delta_{n}\right)^{-1}}^{2}\right)} \mathrm{d} Y
\end{aligned}
$$




$$
\leqslant C \varepsilon R^{m-2} \delta_{n}^{-2} \int_{\substack{B_{\delta_{n}^{-1}}^{2} \times B_{R}^{m-2}\\}}\left(\left|D w_{n}\right|^{2}+\left|\partial_{t} w_{n}\right|^{2}\right)(X, Y, t) \mathrm{d} X \mathrm{~d} Y .
$$

Observe that $\sum_{i j}\left|\mathrm{~d} \omega_{n}^{i} \omega_{n}^{j}-\omega_{n}^{i} \mathrm{~d} \omega_{n}^{j}\right|^{2}=\left|\omega_{n}\right|^{2}\left|D \frac{\omega_{n}}{\left|\omega_{n}\right|}\right|^{2}$ and $\left|\omega_{n}\right| \geqslant \frac{1}{2}$ on $B_{\delta_{n}^{-1}}^{2} \times B_{2 R}^{m-2}$. This, plus integration over $t \in\left(-R^{2}, R^{2}\right)$, yields

$$
\begin{gathered}
\int_{\left(B_{\left(4 \delta_{n}\right)-1}^{2} \backslash B_{2 R}^{2}\right) \times B_{R}^{m-2} \times\left(-R^{2}, R^{2}\right)}\left|D \frac{w_{n}}{\left|w_{n}\right|}\right|^{2}(X, Y, t) \mathrm{d} X \mathrm{~d} Y \mathrm{~d} t \\
\leqslant C \varepsilon R^{m-2} \delta_{n}^{-2} \int_{B_{\delta_{n}^{-1}}^{2} \times B_{R}^{m-2} \times\left(-R^{2}, R^{2}\right)}\left(\left|D w_{n}\right|^{2}+\left|\partial_{t} w_{n}\right|^{2}\right) .
\end{gathered}
$$

Finally, we need to control the $L^{2}$ norm of $D\left|w_{n}\right|$. To do it, write $w_{n}=\rho_{n} \theta_{n}$, with $\rho_{n} \geqslant \frac{1}{2}$ and $\theta_{n}$ valued in $S^{k-1}$, then one has

$$
\Delta \rho_{n}+\varepsilon_{n}^{-2}\left(1-\rho_{n}^{2}\right) \rho_{n}-\rho_{n}\left|D \theta_{n}\right|^{2}=\partial_{t} w_{n} \theta_{n} .
$$

Multiplying both side by $\left(1-\rho_{n}\right)$ and integrating it over $\left(B_{\left(4 \delta_{n}\right)^{-1}}^{2} \backslash B_{2 R}^{2}\right) \times B_{R}^{m-2} \times$ $\left(-R^{2}, R^{2}\right)$, we obtain

$$
\begin{aligned}
& \int_{\left(B_{\left(4 \delta_{n}\right)^{-1}}^{2} \backslash B_{2 R}^{2}\right) \times B_{R}^{m-2} \times\left(-R^{2}, R^{2}\right)}\left|D \rho_{n}\right|^{2} \\
& \leqslant C \varepsilon_{n}^{-2} \int_{B_{\delta_{n}^{-1}}^{2} \times B_{R}^{m-2} \times\left(-R^{2}, R^{2}\right)}\left(1-\rho_{n}^{2}\right)^{2} \\
& +C \int_{\left(B_{\left.\left(4 \delta_{n}\right)^{-1} \backslash B_{2 R}^{2}\right) \times B_{R}^{m-2} \times\left(-R^{2}, R^{2}\right)}^{2}\right.}\left(\left|D \frac{w_{n}}{\left|w_{n}\right|}\right|^{2}+\left|\partial_{t} w_{n}\right|^{2}\right)+\text { boundary terms } \\
& \leqslant C \varepsilon+\mathrm{O}\left(n^{-1}\right) .
\end{aligned}
$$

Here we have used (6.17)-(6.19) to show that the boundary term converges to zero. In particular, we get

$$
R^{-m} \int_{\left(B_{\left(4 \delta_{n}\right)-1}^{2} \backslash B_{2 R}^{2}\right) \times B_{R}^{m-2} \times\left(-R^{2}, R^{2}\right)}\left|D w_{n}\right|^{2}(X, Y, t) \mathrm{d} X \mathrm{~d} Y \mathrm{~d} t \leqslant C \varepsilon .
$$

This, combines with the Allard's strong constancy lemma (see, [23,32]), implies

$$
\int_{\left(X_{n}\right) \backslash B_{2 R}^{2}\left(X_{n}\right)}\left|D w_{n}\right|^{2}\left(X, Y_{n}, t_{n}\right) \mathrm{d} x \leqslant C \varepsilon .
$$

This finishes the proof of Step 2. Therefore the proof of Theorem 6.1 is complete. 
Now we discuss the quantization result at time infinity for sequences of solutions to (1.5)-(1.6). Let $u_{n} \in C^{\infty}\left(\Omega \times R_{+}, R^{k}\right)$ solve (1.5) and (1.6). By adopting the same notations as Section 4, we know that for $V_{u_{n}(t)}=\delta_{A\left(u_{n}\right)(\cdot, t)} e\left(u_{n}\right)(x, t) \mathrm{d} x$,

$$
\begin{aligned}
\delta V_{u_{n}(t)}(X)= & \int_{\Omega} \partial_{t} u_{n}(x, t) D u_{n}(x, t) X(x) \mathrm{d} x \\
& -2 \int_{\Omega} D X: \frac{D u_{n} \otimes D u_{n}}{\left|D u_{n}\right|^{2}} \frac{F\left(u_{n}\right)}{\varepsilon_{n}^{2}}(x, t) \mathrm{d} x
\end{aligned}
$$

for any $X \in C_{0}^{1}\left(\Omega, R^{m}\right)$. Note also that, by (1.6) and Lemma 2.4, we can find $t_{n} \uparrow \infty$ such that

$$
\begin{gathered}
\lim _{n \uparrow \infty} \int_{t_{n}-1}^{t_{n}+1} \int_{\Omega}\left|\partial_{t} u_{n}\right|^{2} \mathrm{~d} x \mathrm{~d} t+\int_{\Omega}\left|\partial_{t} u_{n}\right|^{2}\left(x, t_{n}\right) \mathrm{d} x=0, \\
\lim _{n \uparrow \infty} \int_{\Omega} \frac{1}{\varepsilon_{n}^{2}} F\left(u_{n}\right)\left(x, t_{n}\right) \mathrm{d} x=0 .
\end{gathered}
$$

We may assume that $u_{n}\left(t_{n}\right) \rightarrow u_{\infty}$ weakly in $H^{1}\left(\Omega, R^{m}\right)$,

$$
e\left(u_{n}\right)\left(x, t_{n}\right) \mathrm{d} x \rightarrow \mu_{\infty} \equiv \frac{1}{2}\left|D u_{\infty}\right|^{2}(x) \mathrm{d} x+v_{\infty}
$$

for some nonnegative Radon measure $v_{\infty}$ on $\Omega$. Moreover, $V_{u_{n}\left(t_{n}\right)} \rightarrow V_{\infty}$ in $V_{m-2}^{*}(\Omega)$ so that $\left\|V_{\infty}\right\|=\mu_{\infty}$. It follows from (6.46)-(6.48) that $\delta V_{\infty}=0$. Therefore, (4.16) of Lemma 4.6 implies, for all $a \in \operatorname{spt}\left(\left\|V_{\infty}\right\|\right)$ and $0<r \leqslant R<\operatorname{dist}(a, \partial \Omega)$,

$$
\begin{aligned}
& R^{2-m}\left\|V_{\infty}\right\|\left(B_{R}(a)\right)-r^{2-m}\left\|V_{\infty}\right\|\left(B_{r}(a)\right) \\
& \quad \geqslant \int_{B_{R}(a) \backslash B_{r}(a)}|y-a|^{-m-4}\left|S^{\perp}(y)\right|^{2} \mathrm{~d} V(y, S) .
\end{aligned}
$$

In particular, $\Theta^{m-2}\left(\left\|V_{\infty}\right\|, x\right)$ exists for all $x \in \operatorname{spt}\left(\left\|V_{\infty}\right\|\right)$. Now define

$$
\begin{gathered}
\Sigma_{\infty}^{1}=\left\{x \in \Omega: \Theta^{m-2}\left(\left\|V_{\infty}\right\|, x\right) \geqslant \varepsilon_{1}^{2}\right\}, \\
\Sigma_{\infty}^{2}=\left\{x \in \Omega: \lim _{r \downarrow 0} \lim _{n \uparrow \infty} r^{2-m} \int_{P_{r}\left(x, t_{n}\right)}\left|\partial_{t} u_{n}\right|^{2}(z) \mathrm{d} z>0\right\} .
\end{gathered}
$$

Then, by (6.47) and the Vitali's covering argument, we have

$$
H^{m-2}\left(\Sigma_{\infty}^{1}\right)<\infty, \quad H^{m-2}\left(\Sigma_{\infty}^{2}\right)=0 .
$$

Now we need a slice-type $\varepsilon_{0}$-regularity result. 
Claim 6.5. - There exist $\varepsilon_{1}>0$ and $\delta_{1}>0$ such that for any $x \in \Omega$ if

$$
r^{2-m} \int_{B_{r}(x)} e\left(u_{n}\right)\left(x, t_{n}\right) \mathrm{d} x \leqslant \varepsilon_{1}^{2}, \quad r^{2-m} \int_{P_{r}\left(x, t_{n}\right)}\left|\partial_{t} u_{n}\right|^{2}(z) \mathrm{d} z \leqslant \varepsilon_{1}^{4} .
$$

Then

$$
\left(\delta_{1} r\right)^{2} \sup _{B_{\delta_{1} r}(x)} e\left(u_{n}\right)\left(x, t_{n}\right) \leqslant C \varepsilon_{1}^{2} .
$$

Proof. - It follows from Lemma 2.1 that, for any $t_{n}-r^{2} \leqslant t \leqslant t_{n}$

$$
\begin{aligned}
& r^{2-m} \int_{B_{r}(x)} e\left(u_{n}\right)\left(x, t_{n}\right)-r^{2-m} \int_{B_{\frac{r}{2}}(x)} e\left(u_{n}\right)(x, t) \\
& \geqslant-r^{2-m} \int_{P_{r}\left(x, t_{n}\right)}\left|\partial_{t} u_{n}\right|^{2}(z) \mathrm{d} z-C\left(r^{-m} \int_{P_{r}\left(x, t_{n}\right)}\left|D u_{n}\right|^{2}\right)^{\frac{1}{2}}\left(r^{2-m} \int_{P_{r}\left(x, t_{n}\right)}\left|\partial_{t} u_{n}\right|^{2}\right)^{\frac{1}{2}} \\
& \geqslant-C \varepsilon_{1}^{2} .
\end{aligned}
$$

Here we have used (3.2). Therefore, we have, for all $t \in\left[t_{n}-r^{2}, t_{n}\right]$

$$
\left(\frac{r}{2}\right)^{2-m} \int_{B_{\frac{r}{2}}(x)} e\left(u_{n}\right)(x, t) \mathrm{d} x \leqslant C \varepsilon_{1}^{2}
$$

so that we have

$$
\left(\frac{r}{2}\right)^{-m} \int_{P_{\frac{r}{2}}\left(x, t_{n}\right)} e\left(u_{n}\right)(z) \mathrm{d} z \leqslant C \varepsilon_{1}^{2}
$$

Therefore, by choosing $\varepsilon_{1}$ sufficiently small and applying Lemma 2.3, we obtain (6.51).

CLAIM 6.6. $-\Sigma_{\infty}=\Sigma_{\infty}^{1} \cup \Sigma_{\infty}^{2}$ is closed and has finite $H^{m-2}$ measure, and $u_{n} \rightarrow u_{\infty}$ in $C_{\text {loc }}^{1}\left(\Omega \backslash \Sigma_{\infty}, R^{k}\right)$.

Proof. - For any $x_{0} \in \Omega \backslash \Sigma_{\infty}$, there exist $r_{0}>0$ and $n_{0} \gg 1$ such that for $n \geqslant n_{0}$

$$
\begin{gathered}
r_{0}^{2-m} \int_{B_{r_{0}}\left(x_{0}\right)} e\left(u_{n}\right)\left(x, t_{n}\right) \mathrm{d} x \leqslant r_{0}^{2-m}\left\|V_{\infty}\right\|\left(B_{r_{0}}\left(x_{0}\right)\right)+\varepsilon_{1}^{2} \leqslant 2 \varepsilon_{1}^{2}, \\
r_{0}^{2-m} \int_{P_{r_{0}}\left(x_{0}, t_{n}\right)}\left|\partial_{t} u_{n}\right|^{2}(z) \mathrm{d} z \leqslant \varepsilon_{1}^{4} .
\end{gathered}
$$

Therefore, Claim 6.5 implies that for $n \geqslant n_{0}$, $\sup _{B_{\delta_{1} r_{0}}\left(x_{0}\right)} e\left(u_{n}\right)\left(x, t_{n}\right) \leqslant C \varepsilon_{1}^{2}$. Hence $B_{\delta_{1} r_{0}}\left(x_{0}\right) \cap \Sigma_{\infty}=\emptyset$ so that $\Sigma_{\infty}$ is closed and $u_{n} \rightarrow u_{\infty}$ in $C_{\text {loc }}^{1}\left(\Omega \backslash \Sigma, R^{k}\right)$. Note also that this and (6.47) imply that $u_{\infty}$ is a weakly harmonic map whose singular set is contained in $\Sigma_{\infty}$. Now we are ready to state the energy quantization theorem at time infinity. 
THEOREM 6.7. - Under the same notations as above. We have (1) $\Sigma_{\infty}$ is a closed $(m-2)$-rectifiable set. (2) If, in addition, that $N=S^{k-1}$. Then, for $H^{m-2}$ a.e. $x \in \Sigma_{\infty}$, there exist $1 \leqslant l_{x} \leqslant \frac{E_{0}}{\varepsilon_{0}^{2}}$ and $l_{x}$-many harmonic $S^{2}{ }^{\prime},\left\{\phi_{j}\right\}_{j=1}^{l_{x}}$, such that

$$
\Theta^{m-2}\left(\left\|V_{\infty}\right\|, x\right)=\sum_{j=1}^{l_{x}} E\left(\phi_{j}, S^{2}\right) .
$$

Proof. - (1) follows from the fact that $V_{\infty}$ is stationary and Theorem 4.9. (2) It is very similar to that of Theorem 6.1. One can also view it as a $L^{2}$-perturbation argument of that of [32]. The only difference we need to make is to replace (6.7) by the following: for $H^{m-2}$ a.e. $x_{0} \in \Sigma_{\infty}$,

$$
\lim _{r \downarrow 0} \lim _{n \uparrow \infty} r^{2-m} \int_{B_{r}\left(x_{0}\right)}\left|D_{T} u_{n}\right|^{2}(x) \mathrm{d} x=0,
$$

for all $T \in T_{x_{0}} \Sigma_{\infty}$. (6.53) follows from the $H^{m-2}$-approximate continuity of $\Theta^{m-2}\left(\left\|V_{\infty}\right\|, \cdot\right)$ at $x_{0}$ and the monotonicity inequality (6.49) (one can see Lemma 2.4 of [23] or $\$ 3$ of [32]). Then one can follows lines by lines of the proof of theorem 6.1 to show (6.52).

\section{Final remarks}

In this section, we consider the class $\mathcal{A}$ consisting all of the weak solutions $u \in$ $H_{\text {loc }}^{1}\left(\Omega \times R_{+}, N\right) \cap L^{\infty}\left(R_{+}, H^{1}(\Omega, N)\right)$ to the heat equation of harmonic maps (1.0), which satisfy (1) the Pohozaev identity: (cf. also (5.19))

$$
\int_{\Omega \times R_{+}} \partial_{t} u D u X(x)=\int_{\Omega \times R_{+}} \frac{1}{2}|D u|^{2} \operatorname{div}(X)-\sum_{1 \leqslant i j \leqslant m} u_{i} u_{j} X_{j}^{i}
$$

for any $X \in C_{0}^{1}\left(\Omega, R^{m}\right)$; (2) the energy inequality (2.1); (3) the $\varepsilon_{0}$-regularity Lemma 2.3. Note that the class of weak solutions satisfying both (7.1) and (2.1) was introduced by Feldman [17], which was shown to satisfy Lemma 2.3 for $N=S^{k-1}$ by [10] and [17] independently. Since the partial regularity was not proven for general manifold $N$ yet, we henceforth add the property (3) in the definition of the class $\mathcal{A}$. The goal of this section is to point out that all the results from Sections 3-6 are remaining to be true for the class $\mathcal{A}$, and the proofs are almost the same or slightly easier.

As calculated in [17], any $u \in \mathcal{A}$ satisfies the energy monotonicity inequality (2.3) and (2.4) with $e\left(u_{\varepsilon}\right)$ replaced by $\frac{1}{2}|D u|^{2}$ and $F\left(u_{\varepsilon}\right)$ replaced by 0 . Now suppose that $\left\{u_{n}\right\} \subset \mathcal{A}$ satisfy the same initial value $u(x, 0)=u_{0}(x)$ for a $u_{0} \in C^{1}(\Omega, N)$

$$
\sup _{0<t<\infty}\left(\int_{0}^{t} \int_{\Omega}\left|\partial_{t} u_{n}\right|^{2}+E\left(u_{n}(\cdot, t)\right)\right) \leqslant E\left(u_{0}\right) .
$$


Then, as before, one assumes

$$
\begin{gathered}
\frac{1}{2}\left|D u_{n}\right|^{2}(x, t) \mathrm{d} x \mathrm{~d} t \rightarrow \frac{1}{2}|D u|^{2}(x, t) \mathrm{d} x \mathrm{~d} t+v \equiv \mu, \\
\left|\partial_{t} u_{n}\right|^{2}(x, t) \mathrm{d} x \mathrm{~d} t \rightarrow\left|\partial_{t} u\right|^{2}(x, t) \mathrm{d} x \mathrm{~d} t+\eta
\end{gathered}
$$

for two nonnegative Radon measures $v=v_{t} \mathrm{~d} t$ and $\eta$ on $\Omega \times R_{+}$. If we define $\Sigma$ as in Section 2, with $e\left(u_{n}\right)$ replaced by $\frac{1}{2}\left|D u_{n}\right|^{2}$, then Facts 2.4-2.11 all remain to be true.

For $z_{0} \in \Sigma$, one can consider the tangent cone measure space, $T_{z_{0}}(\mu)$, the same way as in Section 3 and Lemma 3.2-Proposition 3.4 remain to hold. In particular, one can define, exactly as same as Definition 3.5, $\operatorname{dim}\left(\Theta^{m}\left(\mu^{0}, \cdot\right)\right)$ for any $\mu^{0} \in T_{z_{0}}(\mu)$. Therefore, we can obtain the same stratification for $\Sigma$ as in Theorem 3.6, namely

THEOREM 7.1. - For any sequence $u_{n} \subset \mathcal{A}$ as above. Let

$$
\Sigma_{k}=\left\{z_{0} \in \Sigma: \operatorname{dim} \Theta^{m}\left(\mu^{0}, \cdot\right) \leqslant k, \forall \mu^{0} \in T_{z_{0}}(\mu)\right\} \quad \text { for } 0 \leqslant k \leqslant m .
$$

Then $\operatorname{dim}\left(\Sigma_{k}\right) \leqslant k$ for $0 \leqslant k \leqslant m$ and $\Sigma_{0}$ is discrete.

One can also associate a generalized $(m-2)$-varifold $V_{u_{n}}$ for each $u_{n}$ as in Section 4. If we let $V$ denote the generalized varifold limit of $V_{u_{n}}$, then all the results from Section 4 remain to be true for $V$. In particular,

THEOREM 7.2. - For $L^{1}$ a.e. $t \in R_{+}, V_{t} \mathrm{~L}\left(\Sigma_{t} \times A_{m-2, m}\right)$ is a $(m-2)$-rectifiable varifold. In particular, $\Sigma_{t}$ is a $(m-2)$-rectifiable set.

For the generalized varifold flow, all the results from Section 5 remain true for $\mathcal{A}$. For example, we have

THEOREM 7.3. - Under the same notations as above. If, in addition, $u$ is a suitable weak solution to the heat equation of harmonic maps. Then $\left\{v_{t}\right\}_{t \geqslant 0}$ is a Brakke flow.

Finally, we can prove an energy quantization for the density function of $V_{t}$ as follows. The proof is similar to that of Theorem 6.1 and in fact is slightly easier. One can also modify the proof of [27] to show the following result.

THEOREM 7.4. - If, in addition, that $N=S^{k-1}$. Then, for $\mathcal{P}^{m}$ a.e $z_{0} \in \Sigma$,

$$
\Theta^{m-2}\left(\left\|V_{t_{0}}\right\|, x_{0}\right)=\sum_{j=1}^{l_{x_{0}}} E\left(\phi_{j}, S^{2}\right)
$$

for some $1 \leqslant l_{x_{0}}<\infty$ and $\{\phi\}_{j=1}^{l_{x_{0}}}$ harmonic $S^{2}$, .

Remark 7.5.-We conjecture that Theorems 6.1, 6.7, and 7.4 are true for any Riemannian manifold $N$.

Remark 7.6. - We believe that the concentration set $\Sigma$ is also $(m-1)$-rectifiable set with respect to the Euclidean metric on $M \times R_{+}$. The stratification Theorem 3.6 may be useful to attack this problem. 


\section{Acknowledgement}

The paper was finalized during the second author's visit to the Courant Institute of Mathematical Sciences in the Fall 1999, and to the Department of Mathematics at Princeton University in the Spring 2000. The second author wishes to express his gratitude to both institutions for their hospitalities and Professor Alice Chang and Pual Yang at Princeton University for their interests.

\section{REFERENCES}

[1] Ambrosio L., Soner H.M., A measure-theoretic approach to higher codimension mean curvature flows (Dedicated to Ennio De Giorgi), Ann. Scuola Norm. Sup. Pisa Cl. Sci. (4) 25 (1-2) (1997) 27-49 (1998).

[2] Ambrosio L., Soner H.M., Level set approach to mean curvature flow in arbitrary codimension, J. Differential Geom. 43 (4) (1996) 693-737.

[3] Almgren F.J.Jr., $Q$ valued functions minimizing Dirichlet's integral and the regularity of area minimizing rectifiable currents up to codimension two, Bull. Amer. Math. Soc. (N.S.) 8 (2) (1983) 327-328.

[4] Almgren F.J.Jr., The Theory of Varifolds. Mimeographed Notes, Princeton, 1965.

[5] Allard W.K., An integrality theorem and a regularity theorem for surfaces whose first variation with respect to a parametric elliptic integrand is controlled, in: Geometric Measure Theory and the Calculus of Variations, Arcata, CA, 1984, Proc. Sympos. Pure Math., Vol. 44, American Mathematical Society, Providence, RI, 1986, pp. 1-28.

[6] Allard W.K., On the first variation of a varifold, Ann. of Math. (2) 95 (1972) 417-491.

[7] Bethuel F., On the singular set of stationary harmonic maps, Manu. Math. 78 (4) (1993) 417-443.

[8] Brakke K., The Motion of a Surface by its Mean Curvature, Mathematical Notes, Vol. 20, Princeton University Press, Princeton, NJ, 1978.

[9] Cheng X.X., Estimate of the singular set of the evolution problem for harmonic maps, J. Differential Geom. 34 (1) (1991) 169-174.

[10] Chen Y.M., Li J.Y., Lin F.H., Partial regularity for weak heat flows into spheres, Comm. Pure Appl. Math. 48 (4) (1995) 429-448.

[11] Chen Y.M., Lin F.H., Evolution of harmonic maps with Dirichlet boundary conditions, Comm. Anal. Geom. 1 (3-4) (1993) 327-346.

[12] Caffarelli L., Kohn R., Nirenberg L., Partial regularity of suitable weak solutions of the Navier-Stokes equations, Comm. Pure Appl. Math. 35 (6) (1982) 771-831.

[13] Chen Y.M., Struwe M., Existence and partial regularity results for the heat flow for harmonic maps, Math. Z. 201 (1) (1989) 83-103.

[14] Ding W.Y., Tian G., Energy identity for a class of approximate harmonic maps from surfaces, Comm. Anal. Geom. 3 (3-4) (1995) 543-554.

[15] Eells J., Sampson J., Harmonic mappings of riemannian manifolds, Amer. J. Math. 86 (1964) 109-160.

[16] Federer H., Geometric Measure Theory, Springer-Verlag, New York, 1969.

[17] Feldman M., Partial regularity for harmonic maps of evolution into spheres, Comm. Partial Differential Equations 19 (5-6) (1994) 761-790.

[18] Federer H., Ziemer W.P., The Lebesgue set of a function whose distribution derivatives are $p$ th power summable, Indiana Univ. Math. J. 22 (1972/73) 139-158. 
[19] Helein F., Regularite des applications faiblement harmoniques entre une surface et une variete riemannienne, C. R. Acad. Sci. Paris Ser. I Math. 312 (8) (1991) 591-596.

[20] Ilmanen T., Convergence of the Allen-Cahn equation to Brakke's motion by mean curvature, J. Differential Geom. 38 (2) (1993) 417-461.

[21] Ilmanen T., Elliptic regularization and partial regularity for motion by mean curvature, Mem. Amer. Math. Soc. 108 (520) (1994).

[22] Jerrard R., Soner H.M., Scaling limits and regularity results for a class of Ginzburg-Landau systems, Ann. Inst. H. Poincaré Anal. Non Lineaire 16 (4) (1999) 423-466.

[23] Lin F.H., Gradient estimates and blow-up analysis for stationary harmonic maps, Ann. of Math. (2) 149 (3) (1999) 785-829.

[24] Lin F.H., Mapping problems, fundamental groups and defect measures, Acta Math. Sin. (Engl. Ser.) 15 (1) (1999) 25-52.

[25] Lin F.H., Complex Ginzburg-Landau equations and dynamics of vortices, filaments, and codimension-2 submanifolds, Comm. Pure Appl. Math. 51 (4) (1998) 385-441.

[26] Lin F.H., Varifold type theory for Sobolev mappings, in: AMS/IP Stud. Adv. Math. 20, American Mathematical Society, Providence, RI, 2001, pp. 423-430.

[27] Lin F.H., Riviere T., Energy quantization for harmonic maps. Duke Math. J. (to appear).

[28] Lin F.H., Riviere T., A quantization property for static Ginzburg-Landau vortices, C.P.A.M. 54 (2) (2001) 206-228.

[29] Li J.Y., Tian G., Blow-up Locus for heat flows of harmonic maps, Acta Math. Sin. (Engl. Ser.) 16 (1) (2000) 29-62.

[30] Lin F.H., Wang C.Y., Energy identity of harmonic map flows from surfaces at finite singular time, Calc. Var. Partial Differential Equations 6 (4) (1998) 369-380.

[31] Lin F.H., Wang C.Y., Harmonic and quasi-harmonic spheres, Comm. Anal. Geom. 7 (2) (1999) 397-429.

[32] Lin F.H., Wang C.Y., Harmonic and quasi-harmonic spheres, Part II, Comm. Anal. Geom. (to appear).

[33] Simon L., Lectures on geometric measure theory, Proceedings of the Centre for Mathematical Analysis, Australian National University, 3, Australian National University, Centre for Mathematical Analysis, Canberra, 1983.

[34] Simon L., Theorems on regularity and singularity of energy minimizing maps, Lectures in Mathematics ETH Zürichür, Birkhauser Verlag, Basel, 1996 (based on lecture notes by Norbert Hungerbhler).

[35] Struwe M., On the evolution of harmonic maps in higher dimensions, J. Differential Geom. 28 (3) (1988) 485-502.

[36] Struwe M., On the evolution of harmonic mappings of Riemannian surfaces, Comment. Math. Helv. 60 (4) (1985) 558-581.

[37] White B., Stratification of minimal surfaces, mean curvature flows, and harmonic maps, J. Reine Angew. Math. 488 (1997) 1-35.

[38] Ziemer W.P., Weakly differentiable functions, Sobolev spaces and functions of bounded variation, Graduate Texts in Mathematics, Vol. 120, Springer-Verlag, New York, 1989. 